INL/EXT-21-64567

ANL/NSE-21/65

\title{
Cross Section Generation Capability in Griffin
}

September 2021

Hansol Park ${ }^{1}$, Changho Lee ${ }^{1}$, Yeon Sang Jung ${ }^{1}$, Yaqi Wang ${ }^{2}$, Olin Calvin², Javier Ortensi ${ }^{2}$

${ }^{1}$ Nuclear Science and Engineering Division, Argonne National Laboratory

${ }^{2}$ Nuclear Science and Technology Division, Idaho National Laboratory
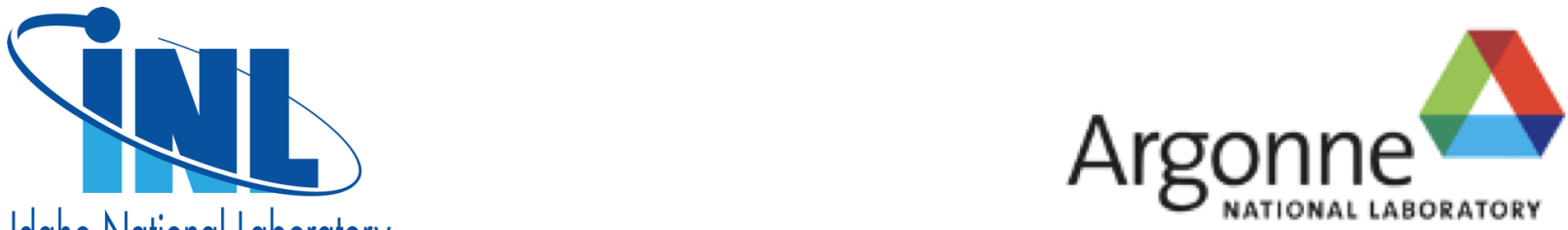

Idaho National Laboratory 


\section{DISCLAIMER}

This information was prepared as an account of work sponsored by an agency of the U.S. Government. Neither the U.S. Government nor any agency thereof, nor any of their employees, makes any warranty, expressed or implied, or assumes any legal liability or responsibility for the accuracy, completeness, or usefulness, of any information, apparatus, product, or process disclosed, or represents that its use would not infringe privately owned rights. References herein to any specific commercial product, process, or service by trade name, trademark, manufacturer, or otherwise, does not necessarily constitute or imply its endorsement, recommendation, or favoring by the U.S. Government or any agency thereof. The views and opinions of authors expressed herein do not necessarily state or reflect those of the U.S. Government or any agency thereof. 
INL/EXT-21-64567

ANL/NSE-21/65

\title{
Cross Section Generation Capability in Griffin
}

\author{
Hansol Park ${ }^{1}$, Changho Lee ${ }^{1}$, Yeon Sang Jung ${ }^{1}$, Yaqi Wang ${ }^{2}$, Olin Calvin ${ }^{2}$, Javier Ortensi ${ }^{2}$ \\ ${ }^{1}$ Nuclear Science and Engineering Division, Argonne National Laboratory \\ ${ }^{2}$ Nuclear Science and Technology Division, Idaho National Laboratory
}

September 2021

\section{Argonne National Laboratory Idaho National Laboratory}

Prepared for the U.S. Department of Energy Office of Nuclear Energy Under UChicago Argonne, LLC Contract DE-AC02-06CH11357 Under DOE Idaho Operations Office Contract DE-AC07-05ID14517 
Page intentionally left blank 


\section{ABSTRACT}

The Griffin code is a Multiphysics Object-Oriented Simulation Environment (MOOSE) based reactor multiphysics analysis application jointly developed by Idaho National Laboratory and Argonne National Laboratory. The code includes a variety of steady-state solvers for fixed-source, k-eigenvalue, adjoint, and subcritical multiplication, as well as transient solvers for point-kinetics, improved quasi-static, and spatial dynamics. The code reads multigroup cross sections in the ISOXML format generated from external deterministic or Monte Carlo cross section generation codes.

The implementation of the cross section generation capability in Griffin was initiated last year by plugging in the cross section application programming interface (CSAPI) and reviewing the methodologies for treating particulate fuels. The focus this year was on improving the CSAPI integration and implementing advanced self-shielding methods for applications to advanced reactor problems with TRISO fuels. First, the process for cross section library generation was updated to accurately and rigorously produce isotopic cross section data. Second, the equivalent Dancoff factor cell method performing slowing down calculations on the fly for the resonance treatment was implemented in CSAPI to improve the accuracy of effective multigroup cross sections in the resonance energy range. Third, the iterative local spatial self-shielding method was implemented under the calculation framework of the equivalent Dancoff factor cell method to accurately deal with the double heterogeneity effect of particulate fuel.

The updated CSAPI with the advanced self-shielding methods, together with the cross section libraries generated based on the improved process, were tested for pin-cell, unit-cell, and fuel assembly problems with various resonance self-shielding conditions based on very high temperature reactor, high temperature test reactor, and Empire benchmark cores, indicating that the updated CSAPI in Griffin is able to produce multigroup cross sections accurately and efficiently. We also showed that the methodology worked well for pebble bed fuel from HTR-10, but the capability still needs to be fully integrated into CSAPI. In the future, further benchmark tests will be performed for various thermal reactor core problems, including particulate fuel-based pebble bed reactors. 
Page intentionally left blank 


\section{CONTENTS}

Abstract ......................................................................... ii

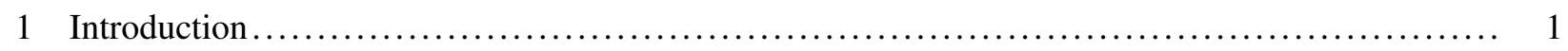

2 Cross Section Capabilities in Griffin ............................................ 4

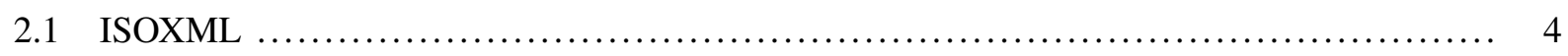

$2.2 \quad$ Generation of the Multigroup Library for CSAPI $\ldots \ldots \ldots \ldots \ldots \ldots \ldots \ldots \ldots \ldots \ldots \ldots \ldots, 6$

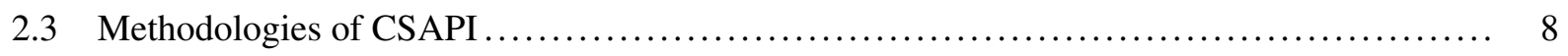

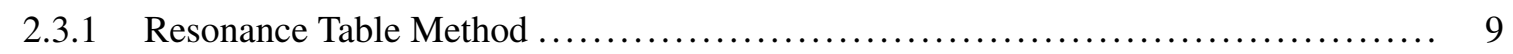

2.3 .2 Equivalent Dancoff Factor Cell (EDC) Method ............................. 13

2.3.3 Iterative Local Spatial Self-shielding (ILSS) Method for Particulate Fuel Modeling .. 15

2.3 .4 Discussion on Online Cross Section Generation $\ldots \ldots \ldots \ldots \ldots \ldots \ldots \ldots \ldots \ldots \ldots \ldots$

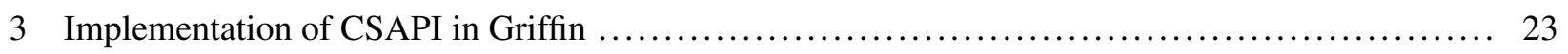

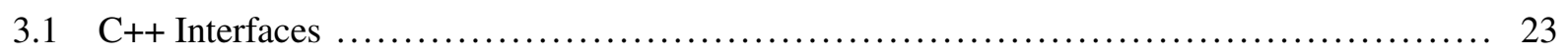

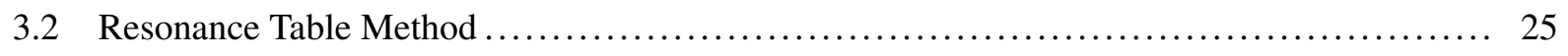

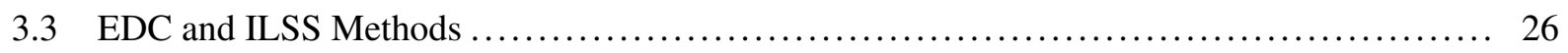

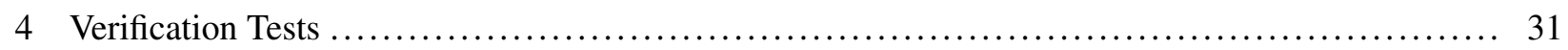

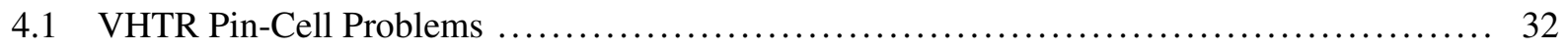

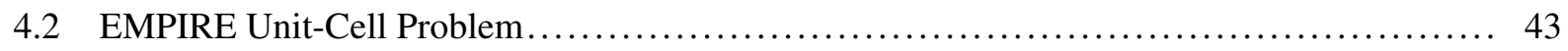

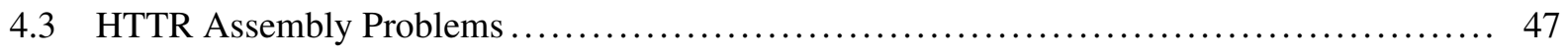

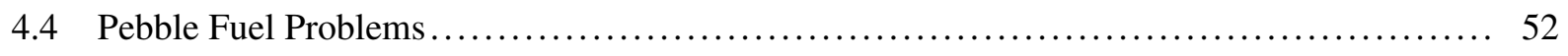

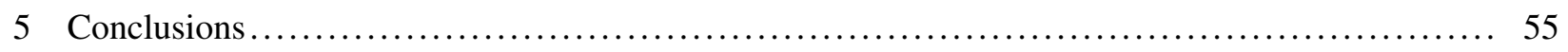

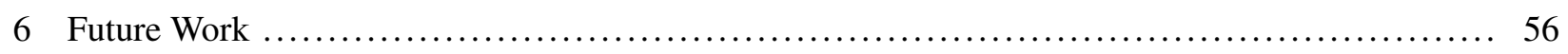

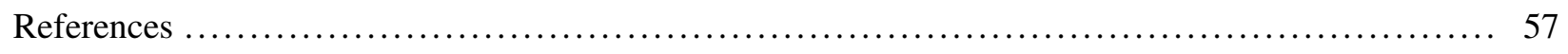




\section{FIGURES}

Figure 1. Process of generating the multigroup library for $\mid$ CSAPI $\mid] \ldots \ldots \ldots \ldots \ldots \ldots \ldots \ldots \ldots \ldots, \quad 9$

Figure 2. Particle cell model to calculate $\mathrm{DF} \beta$ in the resonance energy range. $. . \ldots \ldots \ldots \ldots \ldots \ldots \ldots . \ldots$

Figure 3. Particle cell model to calculate $\mathrm{DF} \beta$ in the fast and thermal energy ranges. ................ 17

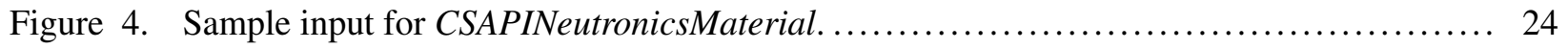

Figure 5. Flowchart of calculating the isotopic background cross sections in the resonance table

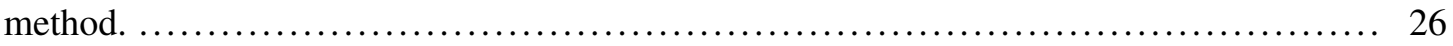

Figure 6. Brief workflow of the $\mid$ EDC|method to generate effective multigroup cross sections ........ 27

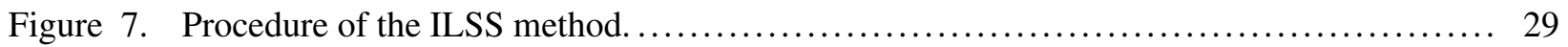

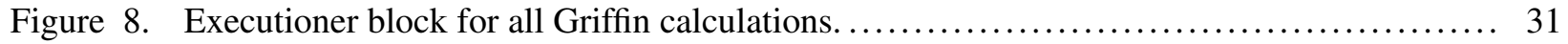

Figure 9. Comparison of lethargy widths of the 68-group structure and the WIMS-69 group structure. 32

Figure 10. Compact design obtained from the $\mid$ NGNP design. $\ldots \ldots \ldots \ldots \ldots \ldots \ldots \ldots \ldots \ldots \ldots \ldots \ldots \ldots \ldots \ldots$

Figure 11. Serpent2 spectra at various fuel-to-moderator volume ratios (at fixed $\mathrm{PS}_{2}$ and $\mathrm{CS}_{2}$ ). ....... 34

Figure 12. Exodus mesh for the VHTR problem of the $\left(\mathrm{CS}_{2}, \mathrm{CM}_{2}\right)$ case. $\ldots \ldots \ldots \ldots \ldots \ldots \ldots \ldots \ldots \ldots \ldots \ldots \ldots \ldots \ldots$

Figure 13. Comparison of base weighting spectrum used for the multigroup library generation and

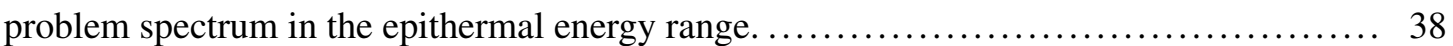

Figure 14. Comparison of spectrum for the $\left(\mathrm{PF}_{3}, \mathrm{CM}_{3}\right)$ case obtained with the multigroup scattering

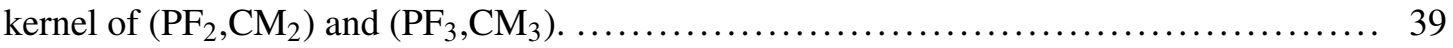

Figure 15. Relative errors of compact-averaged U-238 multigroup capture cross sections for all CS

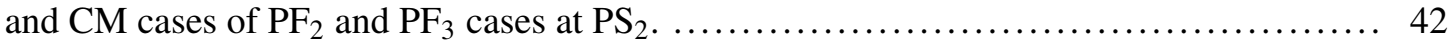

Figure 16. Comparison of Serpent2 spectrum and slowing down spectrum solution of Griffin at 6.67

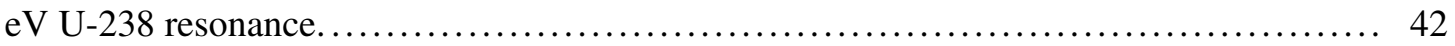

Figure 17. Exodus mesh and material layout of the EMPIRE|2D|unit-cell problem................. 43

Figure 18. Serpent2 spectrum of the fuel compact and the relative error (\%) of Griffin spectrum. ..... 46

Figure 19. Relative errors of compact-averaged multigroup capture cross sections of U-238 in the

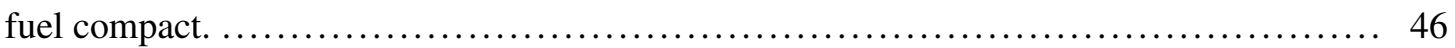

Figure 20. Exodus mesh for the HTTR $2 \mathrm{D}$ assembly problem of $\mathrm{B}_{4} \mathrm{C}$ rodded case. . ............... 47

Figure 21. Dancoff factors and radii of $\mid$ EDC $\$$ of fuel compacts for $2 \mathrm{D}$ |HTTR assembly problem...... 48 Figure 22. Comparison of U-238 capture rates per fission neutron and absolute errors for $\mathrm{B}_{4} \mathrm{C}$ rodded

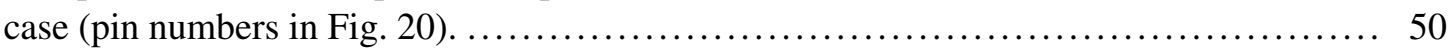

Figure 23. Comparison of pin power distribution and relative errors (\%) for $\mathrm{B}_{4} \mathrm{C}$ rodded case (pin

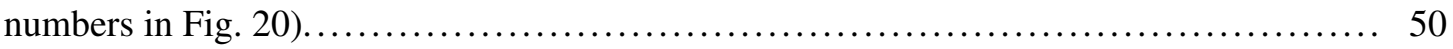

Figure 24. Relative errors of compact-averaged multigroup capture cross sections of U-238 at the

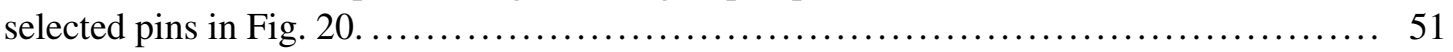

Figure 25. Pebble design obtained from HTR-10 design . . .............................. 53 


\section{TABLES}

Table 1. Eigenvalue results of $\mid$ LWR $\mid$ pin cell problem for the $\mid$ CSAPI $[$ integration test. ............... 25

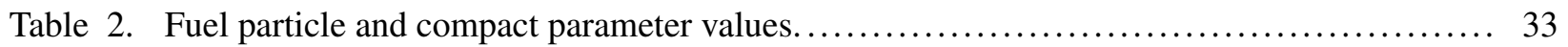

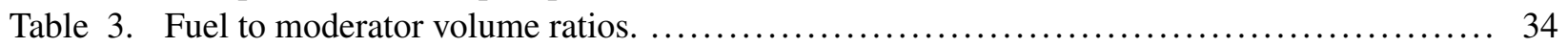

Table 4. Serpent2 eigenvalues of homogeneous (single heterogeneous) and heterogeneous (double heterogeneous) compact problems and TRISO heterogeneity effects in $\mathrm{pcm} . \ldots \ldots \ldots \ldots \ldots .36$

Table 5. Eigenvalue errors (pcm) of the $\mid$ EDC $\mid$ method for single heterogeneous problems. ........... 37

Table 6. $\quad$ Errors of self- and down-scattering kernels of graphite from Group-1 to Group-4 in Fig. 13 due to the use of spectrum with overestimated moderation. $\ldots \ldots \ldots \ldots \ldots \ldots \ldots \ldots \ldots \ldots, 38$

Table 7. Eigenvalue errors ( $\mathrm{pcm}$ ) of the|EDC/method for single heterogeneous problems with update

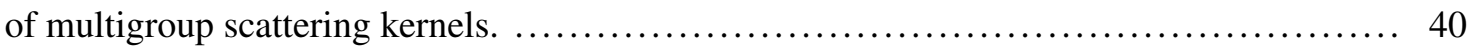

Table 8. Eigenvalue errors $(\mathrm{pcm})$ of the $\mid$ EDC $+\mid$ ILSS $\mid$ method for double heterogeneous problems with update of multigroup scattering kernels. $\ldots \ldots \ldots \ldots \ldots \ldots \ldots \ldots \ldots \ldots \ldots \ldots \ldots \ldots \ldots, 40$

Table 9. Errors (pcm) in TRISO heterogeneity effect of the ILSS $\mid$ method (Table 8 - Table 7). ........ 40

Table 10. Eigenvalue results of Griffin compared to Serpent2 for EMPIRE unit-cell problem. ......... 44

Table 11. Two-group reaction rates per fission neutron of Serpent 2 for each region. ................ 45

Table 12. Absolute errors of 2-group reaction rates per fission neutron obtained by the $\mid$ EDC $+\mid$ ILSS

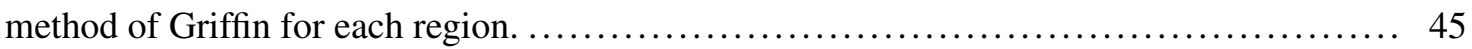

Table 13. Comparison of eigenvalue results for single and double heterogeneous problems of $2 \mathrm{D}$ | HTTR assembly problems with and without $\mathrm{B}_{4} \mathrm{C} \mid \mathrm{BP}$ rods. $\ldots \ldots \ldots \ldots \ldots \ldots \ldots \ldots \ldots \ldots \ldots \ldots$

Table 14. Computation time (seconds) of each task for $2 \mathrm{D} \mid$ HTTR assembly problem with 68 energy

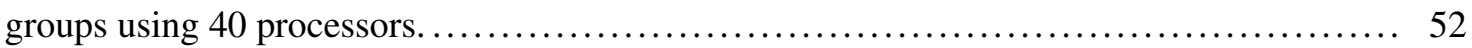

Table 15. Serpent2 results for helium-cooled pebble cell problems with different fuel particle packing

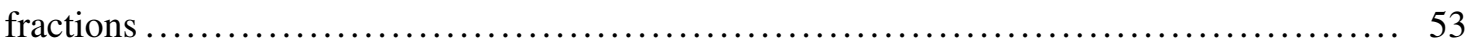

Table 16. Differences (pcm) in eigenvalue and TRISO heterogeneity effect between $\mathrm{MC}^{2}-3$ and Serpent 2 solutions for helium-cooled pebble cell problems. 


\section{ACRONYMS}

$\begin{array}{ll}\text { OD } & \text { Zero-dimensional } \\ \text { 1D } & \text { One-dimensional } \\ \text { 2D } & \text { Two-dimensional } \\ \text { 3D } & \text { Three-dimensional } \\ \text { BP } & \text { Burnable Poison } \\ \text { CMFD } & \text { Coarse-Mesh Finite Difference } \\ \text { CPM } & \text { Collision Probability Method } \\ \text { CSAPI } & \text { Cross Section Application Programming Interface } \\ \text { DF } & \text { Disadvantage Factor } \\ \text { DFEM } & \text { Discontinuous Finite Element Method } \\ \text { EDC } & \text { Equivalent Dancoff factor Cell } \\ \text { ENCM } & \text { Enhanced Neutron Current Method } \\ \text { ENDF } & \text { Evaluated Nuclear Data File } \\ \text { ESSM } & \text { Embedded Self-Shielding Method } \\ \text { FSP } & \text { Fixed Source Problem } \\ \text { HFEM } & \text { Hybrid Finite Element Method } \\ \text { HFG } & \text { Hyperfine Group } \\ \text { HTTR } & \text { High Temperature Test Reactor } \\ \text { ILSS } & \text { Iterative Local Spatial Self-shielding } \\ \text { LWR } & \text { Light Water Reactor } \\ \text { MOC } & \text { Method of Characteristics } \\ \text { MOOSE } & \text { Multiphysics Object-oriented Simulation Environment } \\ \text { MPI } & \text { Message Passing Interface } \\ \text { NGNP } & \text { Next Generation Nuclear Plant } \\ \text { NR } & \text { Narrow Resonance } \\ \text { Pebble Tracking Transport } \\ \text { PWurized Water Reactor } \\ \text { PW }\end{array}$


$\begin{array}{ll}\text { S-P } & \text { Sanchez-Pomraning } \\ \text { SN } & \text { Discrete Ordinates } \\ \text { SPH } & \text { Super-homogenization } \\ \text { VHTR } & \text { Very High Temperature Reactor }\end{array}$ 
Page intentionally left blank 


\section{Introduction}

The DOE-NE Nuclear Energy Advanced Modeling and Simulation (NEAMS) program has developed a number of advanced modeling and simulation tools to deploy advanced nuclear energy technologies for industry and government organizations. As part of the effort, a Multiphysics Object-Oriented Simulation Environment (MOOSE)-based reactor physics code for multiphysics applications, named Griffin [1], has been developed jointly by Idaho National Laboratory (INL) and Argonne National Laboratory (ANL), which includes a variety of deterministic steady-state transport solvers for fixed source, k-eigenvalue, adjoint, and subcritical multiplication as well as transient solvers for point-kinetics, improved quasi-static, and spatial dynamics.

In this fiscal year, the performance of the transport solvers in Griffin was significantly improved in terms of accuracy and computation time by implementing a discontinuous finite element method (DFEM)-based discrete ordinate $[\mathrm{SN})$ asynchronous transport sweeper [2] together with the coarse mesh finite difference (CMFD) acceleration for heterogeneous- or homogeneous-geometry applications. A new solver based on the hybrid finite element method (HFEM) spherical harmonics expansion (PN) solver (similar to the variational nodal method) [3] for homogeneous-geometry applications was also added. Details were reported in Reference [4].

For transport calculations in Griffin, multigroup cross sections for fast or thermal reactor problems can be provided from an external file in the ISOXML format [5], which are generated from deterministic or Monte Carlo cross section generation codes. Isotopic or composition-based cross section data are stored hierarchically with the natural XML (eXtendable Markup Language) tree structure, which allows a great flexibility to manage isotopic or composition data with many state points.

Last year, to improve the cross section generation capability in Griffin, we initiated the integration of the cross section application programming interface (CSAPI] [6], which is a portable functional module for generating multigroup cross sections using the subgroup or resonance table method for thermal spectrum problems. CSAPI needs a multigroup cross section library, which is generated for a specific reactor or reactor type of interest. This library contains principal isotopic multigroup cross sections as well as resonance cross section data (or subgroup parameters for the subgroup method) as a function of temperature and back-

ground cross section for all isotopes necessary for solving a problem of interest. The CSAPI module by design can be adapted to any transport code with a fixed source solver capability. CSAPI allows transport 
solvers to generate self-shielded multigroup cross sections on the fly (online), accounting for the effects of heterogeneous geometry as well as temperature and composition. Plugging CSAPI into Griffin was made successful by adding the Fortran/C++ interfaces for data transfer between Griffin and CSAPI and putting the DFEM SN-based fixed source solver for escape cross section calculations.

In this fiscal year, the cross section library generation process was updated to better support the advanced resonance self-shielding methods implemented in CSAPI Initially, all isotopic multigroup cross sections including isotopic scattering matrices were generated from OpenMC [7]. However, in order to provide an alternative and verification option to users, the procedure of using the combination of Serpent2 [8] and NJOY [9] was added, in which the two codes produce isotopic principal cross sections and scattering matrices, respectively.

The resonance self-shielding treatment of the CSAPI module in Griffin was also significantly improved with the on-the-fly slowing down method and the particulate fuel modeling capability. Specifically, the equivalent Dancoff factor cell (EDC) method [10, 11] was implemented as the on-the-fly slowing down methods. The EDC method decouples a multidimensional geometry problem (e.g., a whole core) to multiple one-dimensional (1D) pin-cell problems, preserving Dancoff factors of fuel pins calculated in the multidimensional geometry, and solves the hyperfine group $[\mathrm{HFG}, \sim 400,000$ groups) slowing down equation of $\mathrm{MC}^{2}-3$ with the $1 \mathrm{D}$ pin-cell geometry using the collision probability method $(\mathrm{CPM})$. The EDC method takes advantage of the accuracy of the on-the-fly slowing down method without degrading computational time since $1 \mathrm{D}$ pin-cell problems can be solved in parallel using the computationally cheap $1 \mathrm{D}[\mathrm{CPM}$ solver.

Together with the EDC method, the iterative local spatial self-shielding (ILSS) method [12] was introduced for treating the double heterogeneity effect of particulate fuel. The method approximately accounts for the effect of randomly distributed particles on the particle-to-particle shadowing effect using a homogenized composition region surrounding a particle of interest at the center. The cross section of the homogenized composition region should use the self-shielded one of the particle at the center, both of which are therefore determined iteratively. Particle-cell averaged $\mathrm{HFG}$ cross sections are obtained by solving the $\mathrm{HFG}$ slowing down equation with the particle self-shielding iteration in a 1D particle cell problem and used in the EDC method to obtain a weighting spectrum of the stochastic-region for condensation.

The enhanced capability of CSAPI in Griffin was tested for a few reactor problems, including HTTR (High Temperature Test Reactor), VHTR (Very High Temperature Reactor), and Empire microreactor problems. In addition, we extended the tests to a preliminary study with pebble fuel problems, not using the 
Griffin CSAPI but using MC MC $^{2}$ [12, 13], to ensure that the ILSS method implemented in CSAPI worked well for PBR (Pebble Bed Reactor) applications.

In this report, Section 2 introduces the cross section data handling capabilities of the ISOXML module and the cross section generation methodologies of the CSAPI module in Griffin, as well as the CSAPI multigroup cross section library generation procedure. Section 3 presents the implementation of the advanced resonance self-shielding methods to the CSAPI module in Griffin. Verification tests and results are discussed in Section 4 , followed by conclusions and future work in Section 5 and Section 6, respectively. 


\section{Cross Section Capabilities in Griffin}

\subsection{ISOXML}

Nuclear data is provided through evaluated nuclear reaction databases (ENDF] [14-18] stored in the ENDF-6 format [19]. The ENDF-6 rules are defined by the U.S. National Nuclear Data Center (NNDC) on behalf of the Cross Section Evaluation Working Group (CSEWG). An ENDF library can contain data for neutron reactions as well as data relating to radioactive decay, fission-product yield spectrum, neutron thermal scattering, photo-atomic interactions, and more. ENDF libraries are the data source for the modeling and simulation of nuclear reactors.

However, cross section data stored in the ENDF-6 format is not directly usable for radiation transport calculations. As a result, the data in the ENDF-6 format must first be processed by ENDF-6 processing codes such as NJOY [20], AMPX [21], ETOE2 [13], CALENDF [22], FRENDY [23], PREPRO [24], and others. The output of such nuclear data processing codes is either 1) in a multigroup format with resonance self-shielding data, such as GENDF with NJOY, for the deterministic path with lattice physics codes, such as DRAGON [25], MC ${ }^{2}-3$ [13], and TRITON [26], or 2) in an ACE format for continuous energy Monte Carlo codes, such as Serpent [8], MCNP [27], Shift [28], and OpenMC [7]. Both paths can generate reactor-specific multigroup cross section libraries typically without resonance self-shielding data for the final multigroup radiation transport calculations for reactor analysis. The reactor-specific multigroup cross section libraries may require certain equivalence data for regions of interest to account for the spatial homogenization or group condensation effects. The number of energy groups for these multigroup libraries with and without resonance self-shielding/equivalence data can vary from several to thousands. Data used in the deterministic self-shielding codes (Path 1) traditionally employ finer energy group structures (hundreds to thousands of groups) and are tabulated as a function of temperature and other parameters used in the self-shielding methods. Data used in the deterministic full core solvers are typically tabulated with state variables, such as fuel temperature, coolant density, etc., for users to bring in spatial and energy spectrum effects on evaluating local macroscopic cross sections in simulations of engineering-scale geometries. A large number of isotopes, multiple reaction types, and a combination of all state variables can make the multigroup library difficult to manage because of the size of the library. A uniform format for multigroup libraries with resonance self-shielding data/equivalence data for both lattice physics and full-core transport calcula- 
tions is desired. Along with the format, the capability to perform operations on the data (self-shielding, interpolation, mixing, etc.) is also desired for users and developers to avoid repeated coding efforts for these operations.

Existing library formats, like ANISN [29], ISOTXS [30], and AMPX [21], were created decades ago and are obscure to use or do not support all functionalities required by the transport calculations. Lattice physics codes that process the multigroup data from ENDF-6 processing codes have their own input library. Lattice physics codes and Monte Carlo codes also generate multigroup libraries in their own formats. There is a need to modernize these formats and extend their functionalities with the latest available computing tools.

The ISOXML module provides the capabilities to process nuclear data from some of these external cross section generation codes and to produce data in the ISOXML format. ISOXML entails a number of library structures and formats for:

- multigroup cross section and kinetic data

- equivalence data

- decay, transmutation, and fission-product yield data.

The ISOXML format is based on the XML (Extensible Markup Language) [31] format. The multigroup cross section library is designed to manage all isotopes at all state points for any type of calculation (e.g., steady state, transient, and depletion). A state point is described by several state variables (e.g., fuel and coolant temperatures, soluble boron concentration, and accumulated burnup). All of the ISOMXL-formatted libraries share several appealing features:

- the general mark-up language for which robust and efficient parsers exist

- data are managed hierarchically with the natural XML tree structure

- the sequence of nodes in an XML file on the same tree level is irrelevant, which gives the library a great flexibility in managing all state points and all isotopes

- it is human readable and writable, although designed for computer-driven data manipulation, which makes the format very accessible to new users when compared to many other formats

- it allows for complete validity checks. 
As mentioned previously, it bears repeating that the flexibility and readability of the ISOXML data format is one of its most attractive features. Scientific studies frequently need to modify datasets in the process of their modeling, sometimes to remove unneeded data and other times to correct and update data based on recent experimental results. With the $\mathrm{C}++$ object-oriented programming paradigm, the ISOXML format makes it trivial for users to find, modify, and add data to ISOXML libraries, provided that the overall energy group structure of the library is unchanged (which would necessitate generation of a new multigroup library for a new energy spectrum). By increasing the accessibility of the data format to users who were not directly involved in the development of the format, the format (and the data stored in said format) can be more readily accessed, modified, and implemented for modeling and simulation by other users.

To reduce storage requirements, an XML library can be converted to a binary file with netCDF [32]. It needs to be noted beforehand that all symbols in an XML file are case sensitive. A manipulator of ISOXML in C++ with RapidXML [33] is created. A Fortran interface is going to be provided to facilitate coding efforts in Fortran. With this format, operations, including interpolation and mixing, can be done with a uniform interface. Users and developers are shielded from the complexity of these operations.

The ISOXML module is accessible within Griffin but can also be used as a standalone executable. Currently, the ISOXML module can load and convert a number of files to the ISOXML format:

- Serpent2 result files for cross sections and kinetics parameters

- Serpent2 detector files for reference super-homogenization (SPH) flux solutions

- ISOTXS and PMATRX data files

- ORIGEN-formatted decay and fission-product yield data files

- ENDF-6 isotope decay constants, decay chains, and fission yields

- ACE file format for a continuous-energy slowing down solver in Griffin.

\subsection{Generation of the Multigroup Library for CSAPI}

Several computer codes are needed to process cross sections and generate the cross section library for CSAPI Those codes are briefly introduced as follows: 
- OpenMC [7]: a Monte Carlo code to solve a pin cell with an isotope of interest with different number densities and temperatures to produce isotopic cross sections for different background cross section and temperature conditions. OpenMC produces principal cross sections and scattering matrices for isotopes.

- Serpent2 [8]: a Monte Carlo code to solve a pin cell with an isotope of interest to produce isotopic cross sections as OpenMC does. This provides an alternative path of generating isotopic cross sections. Serpent 2 generates principal cross sections for isotopes, but scattering matrices are provided from NJOY since Serpent 2 does not produce them.

- NJOY (Version 2012 or higher) [9]: a nuclear data processing code to produce microscopic cross sections for an isotope of interest. Only fission spectrum and multigroup scattering kernels are generated by NJOY, and scattering matrices are normalized to total scattering cross sections generated from Serpent2.

- GenISOTXS [6]: a code to process the cross sections generated from OpenMC or Serpent 2 to edit out the cross sections in the ISOTXS format or the ASCII format required for the cross section library processing.

- $\mathrm{MC}^{2}-3$ [13]: a multigroup cross section generation code to produce self-shielded cross sections for a given composition. For the cross section library generation, the code reads the fluxes and cross sections generated from a Monte Carlo code and performs the transport calculations using the method of characteristics (MOC) to produce background cross sections for each isotope.

- GenCSLIB [6]: a code to process the data that a Monte Carlo code and $\mathrm{MC}^{2}-3$ generate, tabulate cross sections as a function of background cross section and temperature and produce the cross section library in the ASCII format.

- Python scripts: several Python scripts to extract, verify, and convert data at each calculation step.

Multiple calculation steps are needed to produce the cross section library for CSAPI. The cross section library generation process is summarized below.

- Step 1: run a Monte Carlo code for a pin cell representing a reactor of interest. The calculations are repeated by changing the number density of a resonant isotope of interest such that the background 
cross sections of the resonant isotope vary. As a Monte Carlo code, we used OpenMC [7] since it produces a complete set of microscopic cross sections including scattering matrices (Path 1 in Fig. 1 ). However, microscopic cross sections for some isotopes with small number densities could have too large uncertainties, especially in scattering matrices in the low energy range, even using a very large number of particle histories. As an alternative, Serpent2 and NJOY can be used (Path 2 in Fig. 1), in which principal microscopic cross sections are produced from Serpent2 and scattering matrices are provided from NJOY. In the NJOY calculation, the fine-group spectrum from the pin cell calculation of Serpent 2 may be input to make the group collapsing of scattering matrices consistent with the pin cell calculation. The total scattering cross sections obtained from NJOY are updated to be the same as those from Serpent2.

- Step 2: extract isotopic cross sections from the Monte Carlo outputs, which are used to calculate the background cross sections for resonant isotopes using a deterministic code. We used the MOC solver of $\mathrm{MC}^{2}-3$, solving the fixed source problems $\left(\mathrm{FSP}_{3}\right)$ for all the cases solved by a Monte Carlo code. Additionally, the $\mathrm{SPH}$ factor may be introduced to force the reaction rates from $\mathrm{MC}^{2}-3$ to match those from a Monte Carlo code.

- Step 3: repeat Steps 1 and 2 with different temperatures and then construct a cross section data library for an isotope with the tabulation of fission, absorption, and scattering resonance cross sections in terms of background cross section and temperature. A cross section data library involves higher order scattering matrices as well. All isotopic cross section data libraries are merged to a single file with an index table.

The process of generating the multigroup library for CSAPI is illustrated in Fig. 1, which involves several Python scripts as well as additional computer codes, as shown in the figure, to generate code inputs and process code outputs for linking the calculation steps.

\subsection{Methodologies of CSAPI}

In the Griffin CSAPI, there are two resonance treatment methods available: the resonance table method and EDC method. The former belongs to the Bondarenko approach using a heterogeneous resonance table, and the latter belongs to the on-the-fly slowing down method. For the double heterogeneity treatment, the 


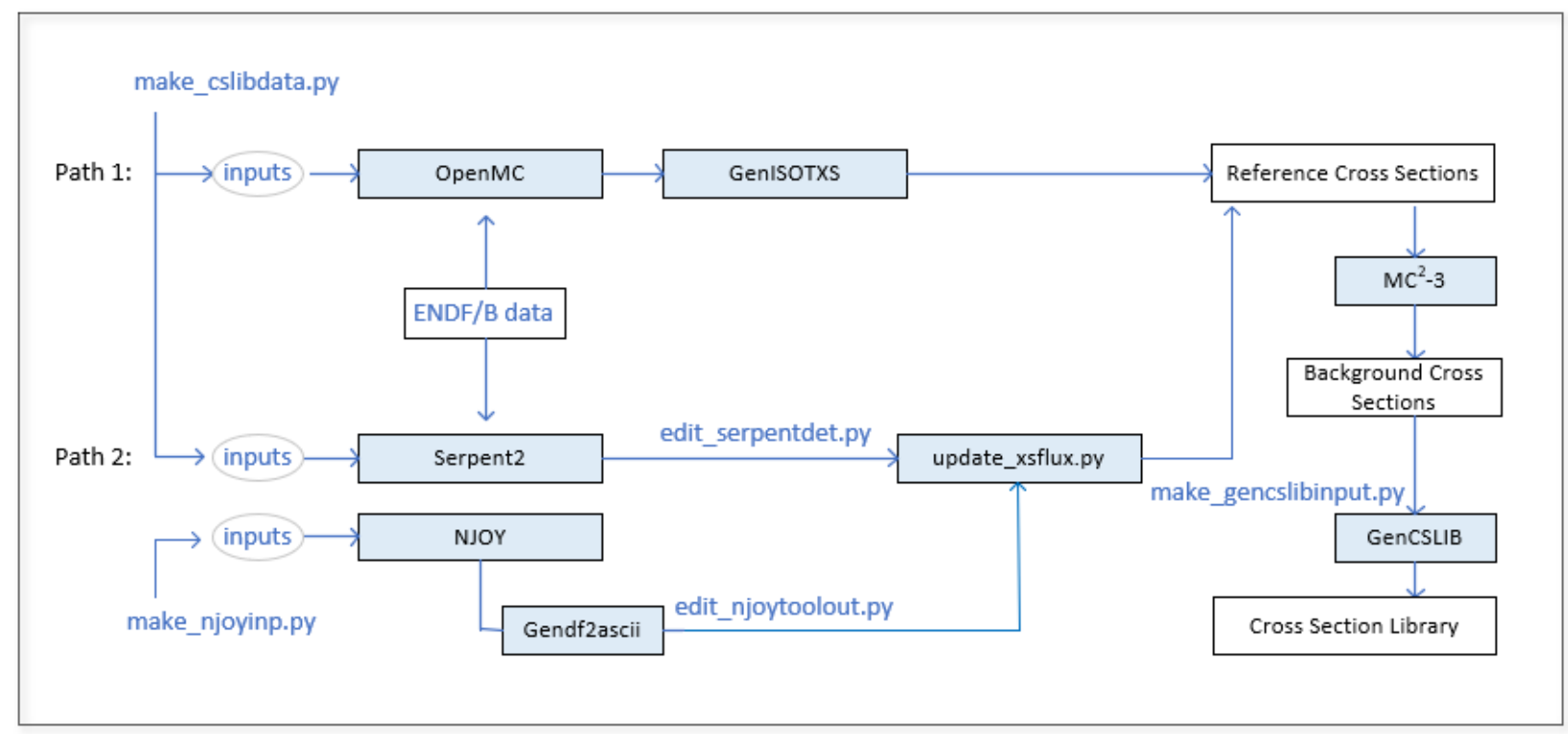

Figure 1: Process of generating the multigroup library for CSAPI

recently developed ILSS method was adopted and incorporated in the calculation framework of the EDC method. These three methodologies are explained in detail in this section.

\subsubsection{Resonance Table Method}

This method is basically the Bondarenko approach [34] that iteratively determines effective cross sections at calculated background cross sections from a pre-generated look-up table. In the conventional equivalence theory [35], resonance integrals are generated by solving slowing down problems in a zerodimensional $0 \mathrm{D}$ problem and tabulated as a function of background cross section and temperature. Then, effective cross sections of resonant isotope $r$ are calculated as

$$
\sigma_{\mathrm{X}, g}^{r}=\frac{R I_{\mathrm{X}, g}^{r}\left(\sigma_{\mathrm{b}, g}^{r}, T\right)}{1-R I_{\mathrm{a}, g}^{r}\left(\sigma_{\mathrm{b}, g}^{r}, T\right) / \sigma_{\mathrm{b}, g}^{r}},
$$

where $\mathrm{X}$ is a reaction type and $\mathrm{a}$ and $\mathrm{b}$ mean absorption and background, respectively. The background cross section typically consists of composition background cross section (volumetric source) and escape cross section (surface source) as

$$
\sigma_{\mathrm{b}, g}^{r}=\frac{\sum_{i \neq r} N^{i} \lambda_{g}^{i} \sigma_{\mathrm{p}}^{i}+D / \bar{l}_{\mathrm{f}}}{N^{r}},
$$


where $r$ and $i$ are isotope indices, p means a potential scattering, $\lambda$ is the intermediate resonance parameter, $N$ means an atomic number density, $D$ is a Dancoff factor, and $\bar{l}_{\mathrm{f}}$ is the mean chord length of a fuel rod. This formulation holds only for a two-region problem (fuel + moderator), based on the narrow resonance (NR) approximation neglecting the resonance scattering and the one-term rational approximation of the escape probability from a fuel rod [35]. The escape probability in the presence of neighboring fuels is corrected via the Dancoff factor, $D$, defined as the probability for a neutron escaping fuel rods in a lattice to have its first collision in non-fuel regions at the black (optical thickness) fuel limit. More advanced formulations use $\mathrm{N}$-terms in the rational approximation of the escape probability and determines $2 \mathrm{~N}$ coefficients of the rational form with the least square fitting at $2 N-1$ grey optical thickness points under the constraint that the sum of $N$ term coefficients equals unity [36].

Meanwhile, the resonance table method in the Griffin CSAPI tabulates the effective cross sections of resonant isotope $r$ directly as a function of background cross section and temperature as:

$$
\sigma_{\mathrm{X}, g}^{r}=\sigma_{\mathrm{X}, g}^{r}\left(\sigma_{\mathrm{b}, g}^{r}, T\right)
$$

where the formula of the background cross section differs from Eq. 2) as will be explained later in this section. The Griffin CSAPI does not rely on the rational approximation of the escape probability. Escape probabilities are implicitly considered when solving an FSP at the region-dependent grey optical thicknesses of fuel rods, and thus this formulation holds in a general geometry where equivalence theory fails.

In addition, this approach also has some parallels with other techniques, such as the physical probability table method of the subgroup method based on a heterogeneous table [37] and the embedded self-shielding method (ESSM) [38, 39] in that the consistency between generating and using heterogeneous tables derived by a user is enforced rather than relying on equivalence theory. The Griffin CSAPI approach is, however, considered to be more advanced than others due to the differences in the formulation of the FSP explained below.

Many lattice physics and whole-core transport codes, such as HELIOS [40], DeCART [41], nTRACER [42], MPACT [43], etc., solve the following FSP for isotope $r$.

$$
\vec{\Omega} \cdot \nabla \psi_{g}^{r}(\vec{x}, \vec{\Omega})+\left(N^{r}(\vec{x}) \sigma_{\mathrm{a}, g}^{r}(\vec{x})+\sum_{i} \lambda_{g}^{i} \Sigma_{\mathrm{p}, g}^{i}(\vec{x})\right) \psi_{g}^{r}(\vec{x}, \vec{\Omega})=\frac{1}{4 \pi} \sum_{i} \lambda_{g}^{i} \Sigma_{\mathrm{p}, g}^{i}(\vec{x}),
$$


where $i$ is an isotope index. Then, the escape cross section for isotope $r$ is calculated as

$$
\sigma_{\mathrm{e}, g}^{r}=\sigma_{\mathrm{a}, g}^{r} \frac{\phi_{g}^{r}}{1-\phi_{g}^{r}}-\frac{1}{N^{r}} \sum_{i} \lambda_{g}^{i} \Sigma_{\mathrm{p}, g}^{i}
$$

where $\phi_{g}^{r}=\int_{\mathscr{S}} \psi_{g}^{r}(\vec{x}, \vec{\Omega}) d \Omega$ with $\mathscr{S}$ being the unit sphere. Although there are slight variations in Eq. (4) among different methods/codes, it should be noted that Eq. (4) ignores the resonance scattering cross section, which means that the true optical thickness is not used. Thus, the physical fuel rod escape probability is not reflected in the flux solution of Eq. (4), resulting in non-negligible errors when directly using equivalence theory. To reduce such errors, the equivalence is enforced between effective (in the pre-generated table) and region-dependent cross sections for a target problem by calculating effective cross sections in the same manner, both in the table generation step and the usage step with a consistent bias. This is called the "enforced equivalence." Due to this nature, the enforced equivalence has a large sensitivity to the representative pin-cell configuration used to generate the table; for example, the enforced equivalence starts to fail in the case that a representative pin-cell and a target pin geometry have different pellet radii [44].

On the other hand, the Griffin CSAPI] uses the formulation of the Tone's method [45] for solving an FSP. The Tone's method was originally developed primarily for the resonance treatment of fast reactor calculations. The advantage of this method is that it does not resort to the rational approximation of the escape probability in equivalence theory, but to more accurate collision probabilities implicitly calculated by solving an FSP. By introducing two key approximations that:

1. the scattering interval of neutrons is much larger than the width of the resonance (narrow resonance [NR] approximation),

2. the energy dependence of the collision probability in an energy group relies only on a target region (i.e., the resonance of a target nuclide within the group is assumed constant in source regions), a spectrum model for resonant isotope $r$ is derived as

$$
\phi_{k}^{r}(E)=\frac{1}{E} \frac{\sigma_{\mathrm{p}}^{r}+\sigma_{\mathrm{b}, \mathrm{p}, k, g}^{r}}{\sigma_{\mathrm{t}, k}^{r}(E)+\sigma_{\mathrm{b}, \mathrm{t}, k, g}^{r}(E)}
$$

where

$$
\sigma_{\mathrm{b}, \mathrm{p}, k, g}^{r}=\frac{\sum_{j} P_{g, j \rightarrow k} V_{j} \sum_{i \neq r} N_{j}^{i} \sigma_{\mathrm{p}}^{i}}{\sum_{j} P_{g, j \rightarrow k} V_{j} N_{j}^{r}} \text { and } \sigma_{\mathrm{b}, \mathrm{t}, k, g}^{r}(E)=\frac{\sum_{j} P_{g, j \rightarrow k} V_{j} \sum_{i \neq r} N_{j}^{i} \sigma_{\mathrm{t}}^{i}(E)}{\sum_{j} P_{g, j \rightarrow k} V_{j} N_{j}^{r}} \text {, }
$$


where $k$ and $j$ are region indices, $i$ is an isotope index, and $\sigma_{\mathrm{b}, \mathrm{p}, k, g}^{r}$ and $\sigma_{\mathrm{b}, \mathrm{t}, k, g}^{r}$ are the potential and total background cross sections, respectively. We adopt the traditional definition for $P_{g, j \rightarrow k}$ as the probability that a neutron born uniformly and isotropically in region $\mathrm{j}$ has its first collision in region $\mathrm{k}$. By further neglecting the energy dependence of the total cross section of other isotopes in the background cross section, $\sigma_{\mathrm{b}, t, k, g}^{r}(E)$, and dividing it into composition background cross section and escape cross section, the final expression of the flux model for isotope $r$ and group $g$ becomes

$$
\phi_{k}^{r}(E) \simeq \frac{1}{E} \frac{\sigma_{\mathrm{p}}^{r}+\sigma_{\mathrm{b}, \mathrm{p}, k, g}^{r}}{\sigma_{\mathrm{t}, k}^{r}(E)+\sigma_{\mathrm{b}, k, g}^{r}}
$$

where

$$
\sigma_{\mathrm{b}, k, g}^{r}=\sigma_{0, k, g}^{r}+\sigma_{\mathrm{e}, k, g}^{r}, \sigma_{0, k, g}^{r}=\frac{\sum_{i \neq r} N_{k}^{i} \sigma_{\mathrm{t}, k, g}^{i}}{N_{k}^{r}} \text { and } \sigma_{\mathrm{e}, k, g}^{r}=\frac{\sum_{j} P_{g, j \rightarrow k} V_{j} \sum_{i \neq r} N_{j}^{i} \sigma_{\mathrm{t}, g}^{i}}{\sum_{j} P_{g, j \rightarrow k} V_{j} N_{j}^{r}}-\sigma_{0, k, g}^{r} \text {. }
$$

In the Griffin CSAPI, the escape cross section in Eq. (9) is evaluated not by directly calculating collision probabilities but by replacing each of the numerator and the denominator with solutions of the following two FSP 3 , respectively, which are solved by the DFEM SN solver in Griffin,

$$
\begin{aligned}
& \text { Numerator: } \vec{\Omega} \cdot \nabla \psi_{g, 1}^{r}(\vec{x}, \vec{\Omega})+\left(\sum_{i} N^{i}(\vec{x}) \sigma_{\mathrm{t}, g}^{i}(\vec{x})\right) \psi_{g, 1}^{r}(\vec{x}, \vec{\Omega})=\frac{1}{4 \pi} \sum_{i \neq r} N^{i}(\vec{x}) \sigma_{\mathrm{t}, g}^{i}(\vec{x}), \\
& \text { Denominator: } \vec{\Omega} \cdot \nabla \psi_{g, 2}^{r}(\vec{x}, \vec{\Omega})+\left(\sum_{i} N^{i}(\vec{x}) \sigma_{\mathrm{t}, g}^{i}(\vec{x})\right) \psi_{g, 2}^{r}(\vec{x}, \vec{\Omega})=\frac{1}{4 \pi} N^{r}(\vec{x}),
\end{aligned}
$$

and

$$
\sigma_{\mathrm{e}, k, g}^{r}=\phi_{k, g, 1}^{r} / \phi_{k, g, 2}^{r}-\sigma_{0, k, g}^{r}
$$

To make this method applicable to equivalence theory, it's assumed that the total cross section of other isotopes is equal to their potential scattering cross section in Eq. (9) to have a consistent background cross section expression and derive the spectrum model equivalent to that in a homogeneous medium. However, since the heterogeneous effective cross section table is employed in the CSAPI library, such an approximation is not required. Instead, the enforced equivalence is imposed as explained previously. It should be noted that the sensitivity of the enforced equivalence to a representative pin-cell configuration would be much smaller than that of the enforced equivalence which resorts to the FSP of Eq. (4). This is because a physically true escape probability is considered in FSP by using best-estimate total cross sections, not ab- 
sorption plus potential cross sections, and the only approximations involved are to neglect the within-group variation of the total cross section of other isotopes in every region, including a target region, and the use of the $\mathrm{NR}$ approximation. The $\mathrm{NR}$ approximation fails in a lower epithermal energy range where resonances are relatively wide, but this bias is covered by the enforced equivalence. Therefore, although not proved explicitly through numerical experiments, the FSP formulation of the Griffin CSAPI would be more robust than that adopted in other lattice physics and whole-core transport codes listed above.

\subsubsection{Equivalent Dancoff Factor Cell (EDC) Method}

This method belongs to the on-the-fly slowing down method, which does not involve the pre-tabulation step. A direct method for this category is to solve the transport equation in the whole domain in a slowing down calculation. A resulting spectrum is used as a weighting function for condensation to the target group structure. However, this approximation-free method is not practical at all, and thus many approximated ways have been proposed [10, 11, 46-48], to which the EDC method belongs. Instead of solving the slowing down problem in an explicit heterogeneous lattice geometry using the DFEM SN solver, the heterogeneous lattice geometry is decoupled to multiple 1D pin-cell problems that preserve the Dancoff factor calculated in the reference geometry using the DFEM $/$ SN solver. The slowing down problem is solved using the $1 \mathrm{D}$ CPM solver, which is computationally very cheap. In the Griffin CSAPI the slowing down calculation scheme of $\mathrm{MC}^{2}-3$ is used [13]; fission and non-elastic scattering sources are assumed to be fixed, and elastic scattering sources are computed recursively on successive HFG (416,400 groups with equal lethargy width of $1 / 24000$ from $14.191 \mathrm{MeV}$ to $0.414 \mathrm{eV}$ ). This approach is basically based on the same idea used in the SCALE procedure that the SCALE Monte Carlo code MCDancoff is used to compute Dancoff factors, which are then used to modify the standard CENTRM two-region[1D unit cell models to account for threedimensional (3D) lattice heterogeneous effects [10]. This approach was successfully demonstrated for a pressurized water reactor (PWR assembly problem by another code named Galaxy as well [11].

A Dancoff factor for each fuel pin is calculated using the enhanced neutron current method (ENCM) [49] by solving the following FSP] once using the DFEM]SN] solver in Griffin.

$$
\vec{\Omega} \cdot \nabla \psi(\vec{x}, \vec{\Omega})+\left(\Sigma_{\mathrm{a}}(\vec{x})+\Sigma_{\mathrm{p}}(\vec{x})\right) \psi(\vec{x}, \vec{\Omega})=\frac{1}{4 \pi} \Sigma_{\mathrm{p}}(\vec{x})
$$

where $\Sigma_{\mathrm{a}}$ is $10^{5}$ for fuel regions and 0 for other regions and $\Sigma_{\mathrm{p}}$ is the potential scattering cross section. A 
Dancoff factor for a fuel lump, $\mathrm{f}$, is calculated as

$$
D_{\mathrm{f}}^{3 \mathrm{D}}=\bar{l}_{\mathrm{f}}\left(\left(\Sigma_{\mathrm{a}, \mathrm{f}}+\Sigma_{\mathrm{p}, \mathrm{f}}\right) \phi_{\mathrm{f}}-\Sigma_{\mathrm{p}, \mathrm{f}}\right),
$$

where $\bar{l}_{\mathrm{f}}$ is the mean chord length of the fuel lump, f. The superscript $3 \mathrm{D}$ means that the $\mathrm{FSP}$ is solved in the whole $3 \mathrm{D}$ geometry. However, a Dancoff factor is typically calculated in a $2 \mathrm{D}$ domain since the resonance treatment is usually performed plane-by-plane without considering the axial leakage effect and the ENCM has not been proved for its applicability to the $3 \mathrm{D}$ geometry. This will be briefly discussed again in Section 2.3.4. Note that all the problems solved in this report are $2 \mathrm{D}$ cases so that this issue is irrelevant to the results in the verification tests. Once the Dancoff factor is obtained, the equivalent outermost radius of $1 \mathrm{D}$ cell of the fuel pin, $R_{\mathrm{eq}}^{1 \mathrm{D}}$, is determined to preserve the Dancoff factor of the original geometry by solving the following non-linear equation using the binary search.

$$
D_{\mathrm{f}}^{1 \mathrm{D}}\left(R_{\mathrm{eq}}^{1 \mathrm{D}}\right)=D_{\mathrm{f}}^{3 \mathrm{D}}
$$

where the left-hand side is the Dancoff factor calculated using the 1D CPM in the 1D cell of which the outermost radius is $R_{\mathrm{eq}}^{1 \mathrm{D}}$. Once the Dancoff-factor equivalent $1 \mathrm{D}$ cell is determined, the fixed source slowing down calculation is performed to obtain the weighting spectrum for condensation.

The approximation in the EDC method poses constraints on the widths of resonance energy groups in mainly two aspects. First, the Dancoff factor approach itself assumes identical fuels in a lattice, and thus, the neighboring effect of different fuel types and temperatures on a within-group spectrum of a target region cannot be considered. Note that the resonance table approach approximately considers this via escape cross sections by using the average magnitude of different total cross sections in neighboring regions in an FSP. Second, the fuel to moderator ratio is not preserved in an EDC, which does not preserve the resonance escape probability, and in turn, the overall spectrum attenuation over energy. For these two reasons, the widths of the resonance energy groups should not be too coarse. Typically, widths similar to those of major U-238 resonances would be the most practical choice as in the WIMS-69 group structure [50]. Coarser group widths would cause notable errors in a weighting spectrum. Note that this constraint on the energy group structure in the resonance energy range also applies to the resonance table method mainly because the replacement of within-group spectrum information with a single parameter of the background cross section 
makes it harder to cover a wide range of variation of the within-group spectrum in larger group widths caused by the resonance interference effect among different isotopes, different lattice heterogeneities, etc.

\subsubsection{Iterative Local Spatial Self-shielding (ILSS) Method for Particulate Fuel Modeling}

For cross section evaluation of particulate fuels, either the transport solver can be modified to consider the microstructure of particles dispersed in a compact or the compact-averaged cross sections are evaluated and passed to the normal transport solver without its modification. In this work, the latter approach was selected and implemented under the calculation framework of the EDC method. By performing slowing down calculations in an appropriate particle cell model, both energy and spatial self-shielding inside particles are taken into account. There are several possible ways to treat particulate fuels for the resonance table method, which will be considered in future work. Currently, particulate fuels can only be modeled with the EDClmethod.

The stochastic region-averaged multigroup microscopic cross section of isotope $i$ in energy group $g$ is computed as

$$
\bar{\sigma}_{\mathrm{X}, g}^{i}=\frac{1}{\bar{N}^{i}} \sum_{k}\left\{N_{k}^{i} v_{k} \frac{\int_{g} \sigma_{\mathrm{X}, k}^{i}(E) \phi_{k}(E) d E}{\int_{g} \bar{\phi}(E) d E}\right\},
$$

where $N_{k}^{i}, v_{k}$, and $\phi_{k}$ are the atomic density of isotope $i$, volume fraction, and flux of micro-region $k$ in a compact, respectively, and $\bar{N}^{i}$ and $\bar{\phi}$ are the average atomic density of isotope $i$ and the average flux in a compact, respectively. To compute the flux at the micro-region $k$, the true ratio of flux at micro-region $k$ to the average flux of the compact is approximated to the ratio obtained in a simplified particle cell model as

$$
\phi_{k}(E)=\frac{\phi_{k}(E)}{\bar{\phi}(E)} \bar{\phi}(E) \approx \frac{\phi_{k}^{\text {p.c. }}(E)}{\bar{\phi}^{\text {p.c. }}(E)} \bar{\phi}^{\text {Lat. }}(E)=d_{k}^{\text {p.c. }}(E) \bar{\phi}^{\text {Lat. }}(E)
$$

where p.c. stands for a particle cell calculation, Lat. means a lattice calculation, and $d$ refers to a disadvantage factor $(\mathrm{DF})$ defined as the ratio of flux at the micro-region to the average flux of the stochastic region. Inserting Eq. (17) into Eq. (16) yields

$$
\bar{\sigma}_{\mathrm{X}, g}^{i}=\frac{1}{\bar{N}^{i}} \sum_{k}\left\{N_{k}^{i} v_{k} \frac{\int_{g} \sigma_{\mathrm{X}, k}^{i}(E) d_{k}^{\text {p.c. }}(E) \bar{\phi}^{\text {Lat. }}(E) d E}{\int_{g} \bar{\phi}^{\text {Lat. }}(E) d E}\right\}=\frac{\int_{g} \bar{\sigma}_{\mathrm{X}}^{i}(E) \bar{\phi}^{\text {Lat. }}(E) d E}{\int_{g} \bar{\phi}^{\text {Lat. }}(E) d E},
$$

where

$$
\bar{\sigma}_{\mathrm{X}}^{i}(E)=\frac{1}{\bar{N}^{i}} \sum_{k} N_{k}^{i} v_{k} \sigma_{\mathrm{X}, k}^{i}(E) \frac{\phi_{k}(E)}{\bar{\phi}(E)} \approx \frac{1}{\bar{N}^{i}} \sum_{k} N_{k}^{i} v_{k} \sigma_{\mathrm{X}, k}^{i}(E) d_{k}^{\mathrm{p} . c .}(E) .
$$


In the energy range where the energy dependence of the cross section and DF cannot be neglected, Eq. 18 is computed in two steps: $d_{k}^{\text {p.c. }}(E)$ is calculated by solving a particle cell problem in the first step, and $\bar{\phi}^{\text {Lat. }}(E)$ is calculated from a lattice self-shielding formalism using the approximated stochastic regionaveraged cross section of Eq. (19) in the second step. In this work, the lattice self-shielding formalism is the EDC method. In the fast and thermal energy range where the energy dependence of the cross section and $\mathrm{DF}$ is neglected, $\sigma_{\mathrm{X}, k}^{i}(E)$ and $d_{k}^{\text {p.c. }}(E)$ are taken out of the integral, and Eq. $\sqrt{18}$ is approximated to

$$
\bar{\sigma}_{\mathrm{X}, g}^{i}=\frac{1}{\bar{N}^{i}} \sum_{k} N_{k}^{i} v_{k} \sigma_{\mathrm{X}, k, g}^{i} d_{k, g}^{\mathrm{p} . \mathrm{c}},
$$

which only requires the $\mathrm{DF}$ calculation that solves a particle cell problem.

The ILSS method [12] is used for calculating DFs. The uniqueness of this method lies in the way that it considers the stochastic effect on spatial self-shielding inside particles. For the resonance energy groups where the mean free path of neutrons is either comparable to or smaller than the average spacing among particles, the stochastic dispersion of neighboring particles should be considered to estimate spatial selfshielding inside particles. For this, a particle of interest at the center is surrounded by the homogenized composition of fuel particles and matrix, including the particle type of interest, as shown in Fig. 2

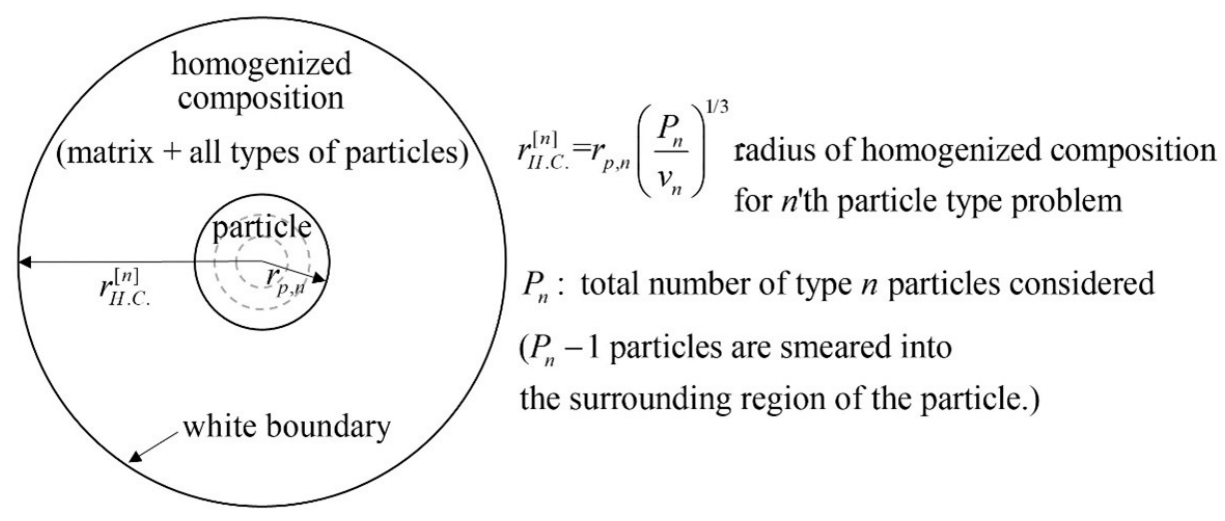

Figure 2: Particle cell model to calculate $\mathrm{DF}$ in the resonance energy range.

The homogenized composition is introduced to model the effect of random particle distribution on the reaction rates in the particle at the center by including the target (center) particle type in it. The most important part is that the homogenized composition cross sections of Fig. 2 use spatially self-shielded cross sections of the particles and matrix, and thus, depend on $\mathrm{DF}$ of them. This makes the $\mathrm{N}$ particle cell problems coupled together, and the coupled set of $\mathrm{N}$ particle cell problems are solved iteratively until all 
$\mathrm{DF}$ converge. Note that, even for $\mathrm{N}=1$, the $\mathrm{DF}$ of the particle should be determined iteratively due to the presence of the $\overline{\mathrm{DF}}$ of the particle type itself in the cross section of the homogenized composition. This DF iteration (i.e., spatial self-shielding iteration) is performed inside the energy loop of the slowing down calculation. Once $d_{k}^{\text {p.c. }}(E)$ is obtained, pointwise stochastic region-averaged cross sections are obtained using Eq. $\left[19\right.$ and used in the lattice self-shielding method to obtain $\bar{\phi}^{\text {Lat. }}(E)$.

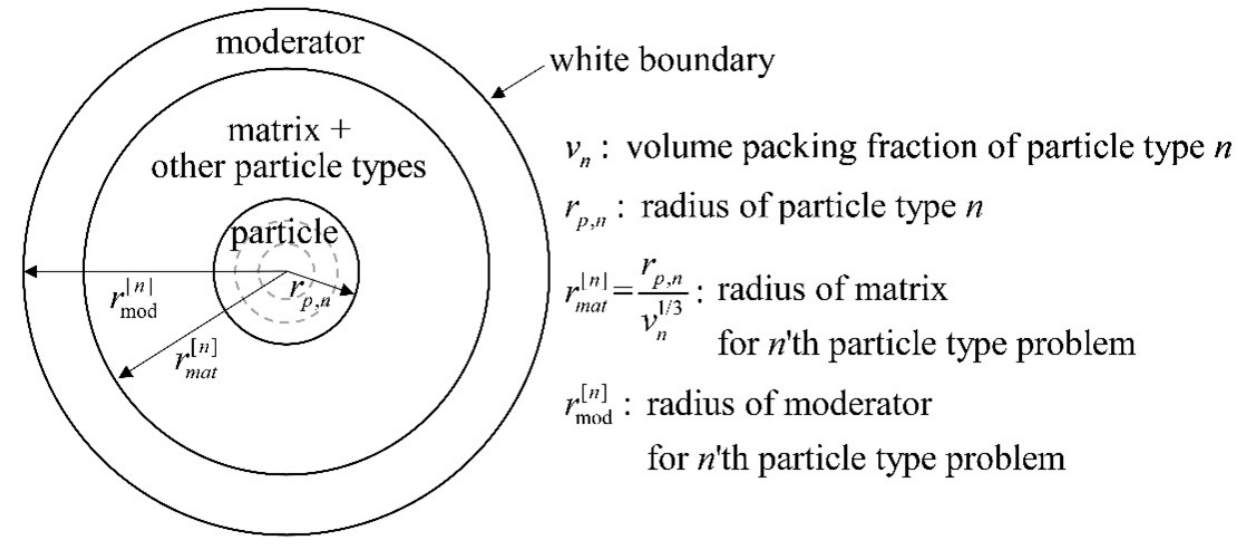

Figure 3: Particle cell model to calculate $\mathrm{DF}$ in the fast and thermal energy ranges.

In the fast energy range, neutrons are born from fission in the fuel kernel and leak to the matrix and the moderator region where neutrons disappear through down-scattering. On top of that, the mean free path of neutrons far exceeds an average spacing among particles and even the distance between compacts. In this circumstance, preserving the relative amount of moderator to fuel is more important than considering the stochastic effect in determining the ratio of the flux in the fuel kernel to the average flux of the compact. Thus, the regular particle array model of Fig. 3 is used instead of that of Fig. 2 . In the thermal energy range, scattering sources from the matrix and the moderator drive the flux distribution inside a compact. In the absence of a large resonance in the fuel kernel, the regular particle array model is accurate enough in determining the $\mathrm{DF}$ in the fuel kernel. The $\mathrm{DF}$ in the fuel kernel is not sensitive to the presence of the moderator at the outside of the matrix in Fig. 3 . Thus, the flux solution of the particle model of Fig. 3 is utilized to compute $\mathrm{DF}$ in the thermal energy range, too. A detailed investigation is needed in future work to check the validity of the particle cell model of Fig. 3 in the thermal energy range for cases such as a plutonium fuel where large resonances in a particle exist. It should be noted that the $\mathrm{N}$ particle-type problems of Fig. 3 are decoupled. Thus, DFs in the fuel particle and matrix are calculated by solving an eigenvalue problem for each particle type in the CSAPImultigroup structure, and stochastic region-averaged multigroup cross sections of Eq. 20] for the fast and thermal energy groups are obtained. 


\subsubsection{Discussion on Online Cross Section Generation}

When generating multigroup cross sections online, there are several points that need to be taken into account and carefully addressed to accurately generate multigroup cross sections for various reactor problems with different conditions.

- Within-group resonance interference effect:

The within-group spectrum is mainly affected by major resonances of resonant isotopes within an energy group. For methods utilizing a pre-tabulated effective cross section set, an error arises from the fact that only resonances of a target resonant isotope are considered in the within-group weighting spectrum used in the generation of the table. The change of the within-group spectrum due to resonances of other resonant isotopes in a target problem is approximately considered by assuming that the resonances of other isotopes are constant within the energy group, and the flux depression due to the resonances of other isotopes occurs uniformly on the average sense in the entire within-group via the background cross section. This causes non-negligible errors, especially in plutonium-dominant regions or in burnable poison or control rod regions that have large resonances in the resonance energy range. On the other hand, the resonance interference effect is naturally accounted for in the on-the-fly slowing down method.

- Non-uniform intra-pellet temperature and burn-up profiles for power reactor:

Intra-pellet variations of temperature and burnup profiles are concerns in power reactors. In order to properly consider this effect in a transport calculation, different cross section zones should be used within a pellet, or properly averaged cross sections over the entire radial zone of the pellet should be used. However, the resonance table approach has two drawbacks. First, when different cross section zones are to be used for radial rings in a pellet, the resonance table approach may have some errors in ring-wise cross sections, mainly because the ring-wise escape probability and the fuel-lump escape probability are different. That is, applying the effective cross section vs. background cross section relation for the fuel lump to ring-wise relations would cause some errors. Note that this holds even for uniform intra-pellet temperature and burnup profile cases. Second, effective cross sections in a resonance table are generated at a uniform intra-pellet temperature and number density, and thus the effect of non-uniform variation within a pellet is missing in the resonance table. There are 
some techniques to approximately consider these effects [51, 52], but those techniques have not been applied in this work yet. Note that the on-the-fly slowing down method is able to naturally consider these effects because different $\mathrm{HFG}$ cross sections at different rings in a pellet are used in the slowing down calculation.

- Thermal up-scattering:

The thermal up-scattering refers to up-scattering due to the thermal agitation of a target nuclide, which gives the secondary energy distribution of double differential scattering kernel the temperature dependency. This temperature dependency of a scattering kernel becomes stronger for heavier nuclides. This is the reason graphite up-scattering is considered to be more important in a graphite-moderated system than hydrogen up-scattering in a LWR. For example, graphite up-scattering can be prominent even at a few tens of eV energy range for temperatures higher than $1000 \mathrm{~K}$. For even heavier nuclides, such as uranium or plutonium isotopes, this temperature dependency is dramatically affected under strongly varying scattering cross sections [53]. Neglecting this effect causes a huge impact on capture rates of $\mathrm{U}-238$ at resonances in the low energy range [54].

In the current CSAPI multigroup library, the multigroup scattering kernel generated using the Serpent 2 + NJOY path has a temperature dependency only up to $10 \mathrm{eV}$ regardless of temperature. This is because NJOY is used to generate the multigroup scattering kernel in the Serpent $2+$ NJOY path, and $10 \mathrm{eV}$ is the maximum incoming neutron energy in the NJOY THERMR module. Another problem is that the NJOY THERMR module does not take into account the resonance scattering in computing the scattering kernel. That is, the free gas scattering from the $\mathrm{S}(\alpha, \beta)$ formulation in the THERMR module is derived under the assumption of the constant scattering cross section [20]. Therefore, work is planned in the next fiscal year to utilize the modification of NJOY made by [55] in which resonance scattering cross section is incorporated in the $S(\alpha, \beta)$ formulation. Updates will be made to the multigroup scattering kernel in the CSAPI multigroup library as well as the $\mathrm{HFG}$ scattering kernel to be used in the slowing down calculation for the EDC method. Note that the effective cross section of the resonance table method takes into account the up-scattering effect since the thermal up-scattering including the resonance up-scattering is accounted for in a Monte Carlo simulation [56, 57].

- Multigroup scattering kernel accounting for problem-dependent spectrum: 
In the multigroup cross section libraries of most lattice physics codes, a multigroup scattering kernel of isotopes does not include a dependency on parameters other than temperature, even though a scattering cross section has a dependency on a background cross section as other reaction cross sections do. In the SCALE code system, a self-scattering has also a dependency on background cross section, and out-going scattering cross sections are determined based on a shielded scattering cross section and a shielded self-scattering cross section [58]. However, the accuracy of the multigroup scattering kernel is mainly determined by the overall slope of a within-group spectrum governed by a fuel-to-moderator ratio and lattice heterogeneity and weakly determined by energy self-shielding due to heavy isotopes in a group. This is very important for a major isotope in a moderator. A multigroup scattering cross section of such an isotope is smooth and does not depend on a within-group spectrum, whereas a pointwise scattering kernel has 1/E dependency over the incoming energy, which significantly affects the multigroup scattering kernel with the slope of the within-group spectrum. Unfortunately, the current CSAPI library does not consider this like most multigroup libraries of lattice physics codes. The need for improvements is demonstrated in the verification section and will be addressed in CSAPI as a future work.

- Angle-dependent multigroup cross section in pin-resolved heterogeneous calculation:

Most of the existing deterministic transport codes for the lattice physics calculation do not use angledependent multigroup total cross sections. For LWR applications, this approximation induces a significant bias in the lattice calculation when explicitly pin-resolved heterogeneous geometry is used. Unlike in the homogenized assembly problem, the angle dependency of multigroup total cross sections in the resonance energy range are large due to a strong variation in the within-group spectrum over angle at the periphery of a fuel rod, the neglect of which causes stronger streaming into the fuel rod and overestimates neutron fluxes in the fuel [59, 60]. This means that an accurate estimation of only the 0th Legendre moment of group cross section in the resonance energy range through the resonance treatment is not sufficient. Such a strong angle dependency of the within-group spectrum does exist in the thermal energy range, but cross sections in the thermal energy range are smooth in a typical energy group structure of lattice physics codes, and group cross sections themselves do not have notable variations over angle in the thermal energy range.

The error caused by only condensing pointwise cross sections with the scalar flux spectrum (the 0th 
moment), not with the angular flux spectrum, is called the condensation error. The transport correction methods have been proposed a long time ago to handle this issue [61], but the target problem was not a pin-resolved heterogeneous transport calculation. Although the efficacy of the method for lattice physics calculations was shown in [59] for a 1D slab geometry, the authors' preliminary studies for a 2D pin-cell geometry revealed several issues in the transport correction method. First, the method requires higher Legendre order moments of the total cross section to obtain transport cross sections. Due to the opposite behavior of the higher order flux spectrum at the vicinity of resonances in the periphery of a fuel rod compared to the scalar flux spectrum, higher order cross sections are an order of magnitude larger than 0th order ones. This may induce instability in the transport solver. Second, there is no resonance treatment theory to estimate such higher order cross sections in pin-resolved heterogeneous geometry.

For these reasons, more simple approaches using equivalence parameters have been used for practicality [46, 60, 62, 65]. The approach using the SPH factor is the most common and easiest to implement. This is often called the multigroup equivalence (between pointwise and multigroup calculations) in French and Japanese literature. As it turned out that the neighboring pin effect on the $\mathrm{SPH}$ factor of a target pin-cell is negligible, it is sufficient to generate the SPH factor at a pin-cell level [60]. Therefore, for the resonance table method in this work, $\mathrm{SPH}$ factors can be generated in the same pin-cell configurations used in generating the effective cross section table over the wide range of background cross sections. That is, $\mathrm{SPH}$ factors can be tallied together with effective cross sections from Serpent2. For the EDC method, SPH factors can be easily calculated on the fly after performing the slowing down calculation in an EDC. In this work, however, this $\mathrm{SPH}$ factor approach to resolve the condensation error has not yet been employed. In fact, this effect is dominant in a normal LWR fuel density, not in low fuel density applications like a graphite-moderated system. A simple test using $M^{2}-3$ for a VHTR pin-cell problem showed that the maximum eigenvalue error induced by the group condensation was at most $30 \mathrm{pcm}$ due to low fuel density, whereas typical condensation errors in an LWR application can cause eigenvalue errors of 200-300 pcm [66] regardless of the energy group structure.

- Axial leakage effect on self-shielding:

Most lattice physics codes do not consider the axial leakage effect in the resonance treatment since 
lattice physics calculations are usually performed on two-dimensional $2 \mathrm{D}$ ) problems. The base theory of the self-shielding treatment is based on a $2 \mathrm{D}$ geometry. In a situation where neutrons can leak out of an axial segment of a fuel rod across its top or bottom plane, the fuel rod escape probability cannot be defined using the mean chord length of a fuel rod because the fuel rod is cut axially, and thus, the formulation in equivalence theory should be re-evaluated. For example, the definition of the Dancoff factor should be altered and the validity of the algorithm for the ENCM should be theoretically verified for such a $3 \mathrm{D}$ case. Nevertheless, the current implementation in the Griffin CSAPI uses the ENCM algorithm for a $3 \mathrm{D}$ geometry, producing seemingly reasonable Dancoff factors in different axial segments of a fuel rod. This would be from the fact that the axial neutron leakage in a fuel rod is negligible due to the black fuel assumption. Efforts are needed to verify this in the next fiscal year. This issue holds for the resonance table method as it should be checked whether the enforced equivalence works or not when using a $2 \mathrm{D}$ table for $\mathrm{a} 3 \mathrm{D}$ application.

- Core-wide spectral transition effect:

Since a direct pin-resolved heterogeneous whole-core calculation in the CSAPI library group structure (>40 groups) is too demanding for the computing resources in general, an appropriate condensation scheme to a broad group level ( $\leq 10$ groups) needs to be devised for practicality, as was done for heterogeneous assembly calculations in the CASMO code [67]. For example, only a local pin-cell calculation is performed with the CSAPI library group structure to obtain a local fine group spectrum. Then, pin/assembly-homogenized problems are solved in the intermediate/broad group structures to obtain the intermediate/broad group spectrum, which are fed back to the local spectrum to obtain the region-wise broad group cross sections and then perform the pin-resolved heterogeneous core calculation in the broad group structure. Appropriate equivalence parameters should be introduced at each condensation/homogenization stage. This should be investigated in future work. 


\section{Implementation of CSAPI in Griffin}

This section focuses on implementation details in Griffin. The CSAPI] module of PROTEUS [68], where

only the resonance table method was available, was ported to Griffin in the last fiscal year [2]. The original Fortran routines in PROTEUS were kept, and the $\mathrm{C}++$ interface was developed by introducing a new material type named CSAPINeutronicsMaterial in Griffin. In this fiscal year, the new resonance treatment methodology of the on-the-fly slowing down approach (the EDC method) and the double heterogeneity treatment capability (the ILSS method) were implemented in the Fortran routines. The first subsection explains the $\mathrm{C}++$ interface part and the implementation schemes of the resonance table method and the EDC and ILSS methods are described in the second subsection.

\subsection{C++ Interfaces}

The CSAPI module was originally developed with Fortran for PROTEUS. Since it was designed and developed to be easily plugged into any heterogeneous transport solvers, the CSAPI module was integrated into Griffin starting from the last fiscal year by:

- Introducing CSAPINeutronicsMaterial to connect the region-wise cross sections computed from CSAPI to the Griffin material systems for transport calculations

- Implementing the Fortran/C++ interface routines to transfer material and region data and cross sections between CSAPI and Griffin

- Plugging the FSP solver (DFEM SN to incorporate the fixed source solver and CSAPI together in order to calculate escape cross sections in CSAPI.

In this fiscal year, further updates have been made in the interfaces and inputs for the CSAPI material type in order to improve its usability. The CSAPI interfaces in Griffin were updated to support the use of multiple CSAPI material blocks in the Griffin input system, as illustrated in Fig. 4, which allows the flexibility of input preparation for complicated material assignments. The FSP solver was also refactored to properly compute the region-wise fluxes based on the mappings of regions and compositions defined in the multiple material blocks. The interface was updated to fully support the parallel execution with distributed meshes for which each processor contains its own partitioned mesh data. Several test problems using CSAPI were added to the Griffin repository for software quality assurance and performance test purposes. 


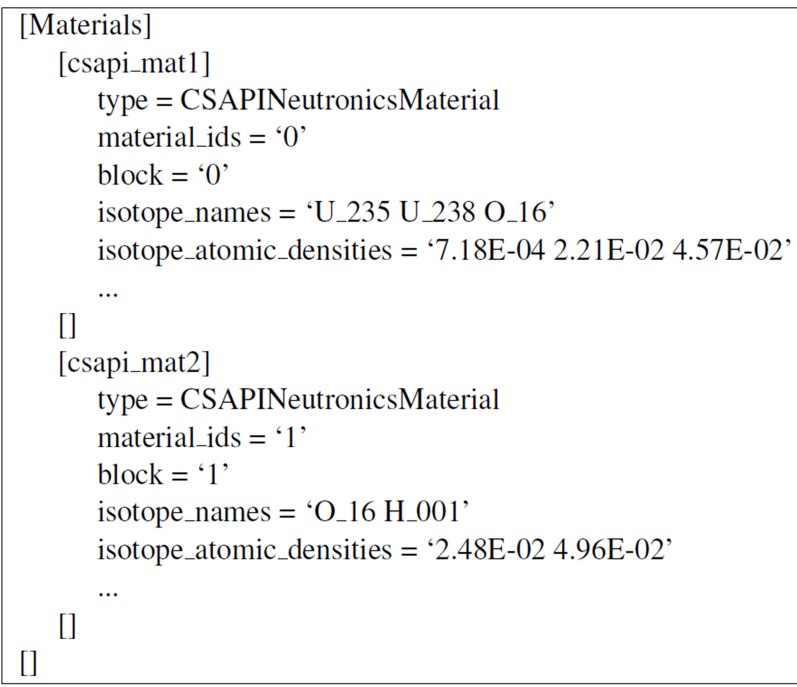

Figure 4: Sample input for CSAPINeutronicsMaterial.

The functionalities of CSAPI were elaborated to perform the robust heterogeneous transport calculations. First, the CSAPI interface to calculate the fission spectrum was updated to properly account for the isotopic data available in the CSAPI library. Previously, the fission spectrum was simply determined for each composition only once at the beginning of the code run by using a uniform spectrum and was not updated by taking into account the spectrum evolution during fission source convergence. This approximation works well without introducing noticeable errors for the thermal reactor case where the fission from U-235 is dominant. However, it can result in non-negligible errors on eigenvalue because fuels for many fission reactors are composed of multiple fissionable isotopes. CSAPINeutronicsMaterial was updated to compute the macroscopic fission spectrum through proper flux-weighting at each iteration to correctly reflect the upto-date solution to the flux-weighting. Second, an interface to compute the fission power was added to the CSAPI material type, which is an essential feature in the reactor analysis. In addition, the CSAPI module was updated to compute a correct kappa-fission value with the spatial self-shielding. The performance of the FSP solver was also improved by removing inefficiencies in memory usage and with various improvements to the sweeper documented in Ref. [4]. The minor bugs in the integration were fixed in the detailed verification work. As shown in Table 1 , the eigenvalues of Griffin and PROTEUS agreed very well within $17 \mathrm{pcm}$. Such a small difference could be caused by discrepancies in the spatial discretization and transport solvers between the two codes (DFEM SN in Griffin and MOC in PROTEUS). 
Table 1: Eigenvalue results of $\mathrm{LWR}$ pin cell problem for the CSAPI integration test.

\begin{tabular}{c|c}
\hline Case & $\Delta k_{\text {eff }}$ (Griffin - PROTEUS) in pcm \\
\hline $\mathrm{UO}_{2}$ Pin & 13 \\
MOX Pin & 12 \\
$\mathrm{UO}_{2} /$ MOX Checkerboard & -17 \\
\hline
\end{tabular}

\subsection{Resonance Table Method}

In order to calculate effective cross sections using Eqs. (3) and (9), escape cross sections need to be evaluated first. The flow diagram to compute escape cross sections of all resonant isotopes is shown in Fig. 5. An energy group is the outermost loop. For each energy group, effective group cross sections of all resonant isotopes are iteratively solved since they are all coupled together through Eqs. (3) and (9). Effective group cross sections are first evaluated at the infinite dilute condition. Then, background cross sections of all resonant isotopes with zero initial values of escape cross sections are calculated using Eq. (9), which are utilized to update effective total cross sections. Once effective total cross sections are determined, the procedure to reevaluate background cross sections starts by solving FSP of each resonance category. In Fig. 5, $C$ means category, which refers to a group of resonant isotopes of similar resonances in that energy group. Special care is needed in categorization, but just a one-to-one relation of isotope and category or element-wise categorization is used in the Griffin CSAPI at the moment. Note that the formulation of FSP in Eqs. (10) and (11) is expressed per isotope. In the category loop, if any isotopes of the current category have resonances in the current energy group, $\mathrm{FSP} \beta$ for the category should be solved. Otherwise, FSP calculations are skipped for the next category. After solving FSP that belong to the category are updated using Eq. (12). Then, background and effective cross sections of all resonant isotopes are updated in the next outer iteration step and the convergence of cross sections is checked. This procedure is repeated until the convergence is satisfied or the maximum number of outer iterations is reached, whichever comes first. In the Griffin CSAPI, the convergence criterion is met when the maximum value of relative differences in total cross sections between successive iteration steps among all resonant isotopes becomes less than $10^{-3}$. The maximum number of outer iterations is set to unity, which is effectively updating the effective cross section twice according to the algorithm of Fig. 5. once before the outer loop and the other with the updated escape cross section outside the outer loop, which is not shown in Fig. 5. It turned out that further updating escape cross sections does not bring practical benefits for the computational effort to solve FSP. 


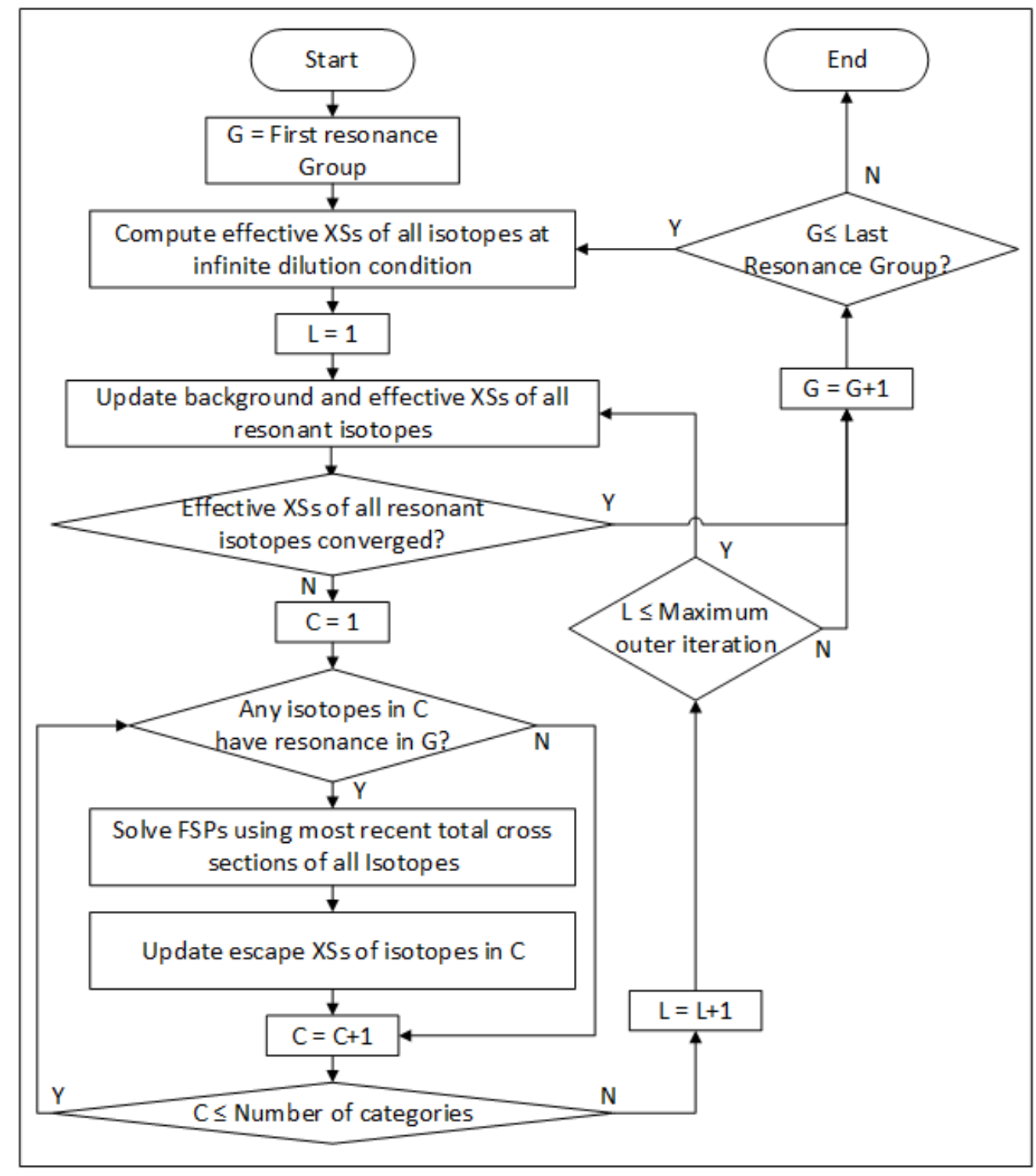

Figure 5: Flowchart of calculating the isotopic background cross sections in the resonance table method.

\subsection{EDC and ILSS Methods}

This subsection explains the implementation details for the EDC and ILSS methods together. Fig. 6 shows the brief workflow. At the beginning, pin-cell geometry and composition are constructed using mesh information from an Exodus mesh as follows:

- To construct pins and internal geometry and compositions of each pin, an extra element integer ID that groups elements into a pin should be present in an Exodus mesh. A user can designate an ID name or pin_id is used. If no such an extra element integer ID is present, the code errors out. 
- For each pin, the centroid point is determined using the centroid point information of each mesh that belongs to the pin. If the pin is cut by symmetry, a user should manually specify centroid points of such pins in an input. This case would be rare.

- For each unique combination of subdomain_id, material_id, and any additional user-specified extra element integer IDs, such as region_id, average distances of elements belonging to each ID set with respect to the centroid of the pin are compared among unique ID sets to determine the geometry and composition of a $1 \mathrm{D}$ cell. The ring radii are calculated by preserving the total volume of elements for each ID set and considering their orders from the center.

- Once pins are constructed, axial segments of pins are examined for whether a fuel region exists or not. Each pin segment that contains a fuel region is designated as an EDC, and EDC $s$ in the whole domain are distributed to MPI (Message Passing Interface) processors.

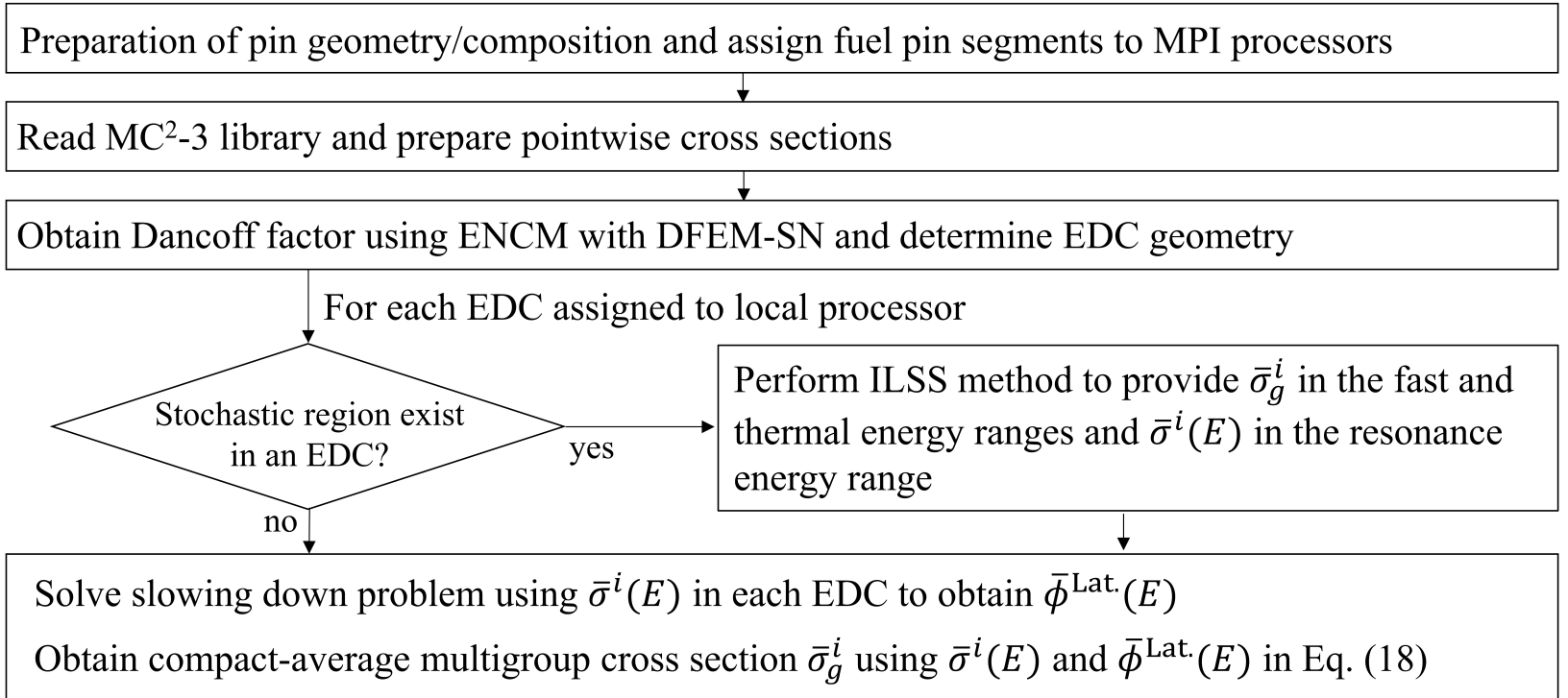

Figure 6: Brief workflow of the $\mathrm{EDC}$ method to generate effective multigroup cross sections

Since the EDC and ILSS methods use the HFG slowing down algorithm of $\mathrm{MC}^{2}-3$, $\mathrm{HFG}$ cross sections are required. Thus, the $\mathrm{MC}^{2}-3$ library data and routines were employed to reconstruct $\mathrm{HFG}$ cross sections. In the process, the followings are accounted for:

- If $\mathrm{HFG}$ cross section files exist in a user-specified directory, data are just read from the files. If they do not exist, $\mathrm{HFG}$ cross sections are reconstructed using the $\mathrm{MC}^{2}-3$ library data and routines. The reconstruction task is distributed to MPI processors for parallel calculations. 
- Since the $\mathrm{MC}^{2}-3$ library and $\mathrm{HFG}$ cross section data require a non-negligible amount of memory, possessing all the data for all MPI processors is not a viable option. Thus, each processor reads only the data of isotopes belonging to it to reconstruct $\mathrm{HFG}$ cross sections and perform the EDC and ILSS calculations.

A Dancoff factor for each pin segment that contains fuel is obtained by solving the FSP of Eq. (13) with the DFEM $[\mathrm{SN}$ solver and using the formula of Eq. (14). Note that the FSP can be solved only once regardless of the number of energy groups since Eq. (13) does not have a group dependency. Then, the outer radius of each fuel segment is determined by solving Eq. (15). For obtaining $D_{\mathrm{f}}^{1 \mathrm{D}}$, the $\mathrm{MC}^{2}-3$ routines to perform the 1DCPM in a cylindrical geometry are used.

Once all EDC $s$ are set up, each processor works on the EDC $\beta$ assigned to it. Before performing the slowing down calculation, the code first checks whether a stochastic region exists in the EDC. If a stochastic region exists, stochastic region-averaged cross sections are prepared by performing the ILSS calculation, whose procedure is shown in Fig. 7. If a stochastic region does not exist in an EDC, the ILSS calculation is just skipped.

- The first step is to calculate stochastic region-averaged multigroup cross sections in the fast and thermal energy range and provide them to the DFEM $S$ transport solver for the transport calculation. An eigenvalue problem is solved using the $1 \mathrm{D} \mid \mathrm{CPM}$ solver in a spherical geometry for a particle cell model of Fig. 3 to obtain multigroup DF. In the particle cell model of Fig. 3 , the outer radius of the moderator region is determined by preserving the fuel to moderator ratio of the EDC where the stochastic region belongs. Multigroup cross sections needed for the eigenvalue calculation are taken from the CSAPI multigroup library. Once multigroup DF are obtained, stochastic region-averaged multigroup cross sections are calculated using Eq. 20) and passed to the DFEM SN transport solver as well as the EDC solver. Note that stochastic region-averaged multigroup cross sections in the resonance energy range calculated in this way are just temporary values and will be updated in the next step. Those temporary values are just used in the EDC calculation initially for calculating fixed sources in the HFG slowing down calculation as explained later.

- The second step is to calculate stochastic region-averaged $\mathrm{HFG}$ cross sections in the resonance energy range and then provide them to the EDC solver for the lattice self-shielding. Since the slowing down 
1) Eigenvalue Problem in Particle Cell of Fig. 3

For particle cell of each particle type

Determine size of moderator region from EDC

Prepare macroscopic multigroup cross sections in the homogenized composition region

Solve eigenvalue problem

Calculate multigroup DFs of all particle types and matrix

Pass $\bar{\sigma}_{g}^{i}$ of Eq.(20) for fast and thermal energy groups to the EDC and DFEM-SN transport solver

2) Slowing-down Problem with DF Iteration in Particle Cell of Fig. 2

For particle cell of each particle type

Prepare macroscopic pointwise cross sections in the homogenized composition region with $\mathrm{DF}=1$

Prepare fixed fission and nonelastic scattering sources

For each energy point

For DF iteration

For particle cell of each particle type

Update macroscopic pointwise cross sections in the homogenized composition region with the most recent DFs

Update collision probabilities

Calculate source

Obtain flux solution and update pointwise DFs of the current particle and matrix

Pass $\bar{\sigma}^{i}(E)$ of Eq.(19) for the resonance energy range to the EDC solver for lattice self-shielding

Figure 7: Procedure of the ILSS method.

calculation scheme is adopted from the $\mathrm{MC}^{2}-3$ formalism [13], the sources from fission and nonelastic (inelastic $+(n, 2 n)$ ) scattering reactions are assumed to be fixed, and only the elastic scattering sources are updated during the slowing down calculation. For each $\mathrm{HFG}$, HFG cross sections in the homogenized composition of Fig. 2 for each particle cell problem of different particle types are updated with the latest HFGDFs of all particle types and matrix. Collision probabilities and sources are updated accordingly, and in turn, flux solutions and $\overline{D F}$ are updated sequentially. This update procedure is iterated until $\mathrm{DF}$ s of all particle types and matrix converge. $\mathrm{DF}$ are assumed to be converged when the maximum value of the relative differences between successive iterations among all particle types and matrix becomes less than $10^{-4}$. Once the last energy group is reached, stochastic region-averaged $\mathrm{HFG}$ cross sections are computed using Eq. (19) and passed to the EDC solver.

Once stochastic region-averaged cross sections are prepared, the EDC method is performed as below: 
- As is done in the ILSS method, the slowing down calculation requires fission and non-elastic scattering sources as fixed sources. For this, the eigenvalue problem is first solved in the EDC geometry. For an EDC with a stochastic region, multigroup cross sections for the eigenvalue problem should be stochastic region-averaged ones computed by the ILSS method. Using the spectrum solution of the eigenvalue problem and stochastic region-averaged fission, inelastic and (n,2n) scattering cross sections, fixed sources are prepared. Then, the slowing down calculation is performed using stochastic region-averaged $\mathrm{HFG}$ total and elastic scattering cross sections. A resulting spectrum is used as a weighting function for group condensation only for the CSAPI library energy groups within the resolved resonance energy range defined in the $\mathrm{MC}^{2}-3$ library. For the unresolved resonance energy range, multigroup cross sections obtained using the resonance table method in the EDC geometry are used. It means that the double heterogeneity treatment is not applied to the unresolved resonance energy range due to its negligible effect in such a high energy range.

- To properly simulate spatial self-shielding inside a fuel near a resonance peak in the EDC geometry, a large enough number of meshes should be used both in fuel and moderator regions. Otherwise, a non-negligible impact is expected on the spectrum solution due to an inaccurate spatial self-shielding. This is done automatically in the code by limiting the maximum optical thickness of each ring $\left(\Sigma_{t} \Delta R\right)$ to 1 and 0.05 for fuel and non-fuel regions, respectively, in the whole slowing down energy range.

Currently, the EDC solver is unable to handle a non-fuel pin that contains resonant isotopes. Since the typical energy range of resonances for light and intermediate mass isotopes is different from that for heavy isotopes, the black optical thickness cannot be assigned to non-fuel regions simultaneously together with fuel regions when constructing EDC 3 . A Dancoff factor for such non-fuel pins could be obtained separately by performing the ENCM calculation one more time, and the average fission sources of fuel pins could be used in the slowing down calculation to obtain a weighting spectrum. The validity of this approach, however, has not been examined. Instead, just the resonance table method is used for resonant isotopes in non-fuel pins, which means that FSP should be solved over the entire domain for resonant isotopes in non-fuel pins. The applicability of the EDC method to non-fuel pins will be examined in future work. 


\section{Verification Tests}

The Griffin CSAPI was verified against the continuous energy Serpent2 [8] Monte Carlo solutions for graphite-moderated thermal reactor problems. First, the prismatic VHTR unit-cell problems over a wide range of pin-cell parameters were solved. Second, the EMPIRE unit-cell problem was solved to check the applicability to heat-pipe-cooled microreactor problems. Third, the HTTR assembly problems with and without $\mathrm{B}_{4} \mathrm{C}$ burnable poison rods $(\overline{\mathrm{BP}} \beta)$ were investigated. All problems discussed here are $2 \mathrm{D}$ problems, and $3 \mathrm{D}$ problems will be addressed in the next fiscal year. All Griffin calculations were performed using the DFEM SN solver with the CMFD acceleration. The Gauss-Chebyshev angular quadrature of three polar and nine azimuthal angles per octant (108 angles for all directions) was used for all Griffin calculations. Coarser meshes for the CMFD calculation were chosen rather than those used for the transport calculation. The Executioner options used are shown in Fig. 8 .

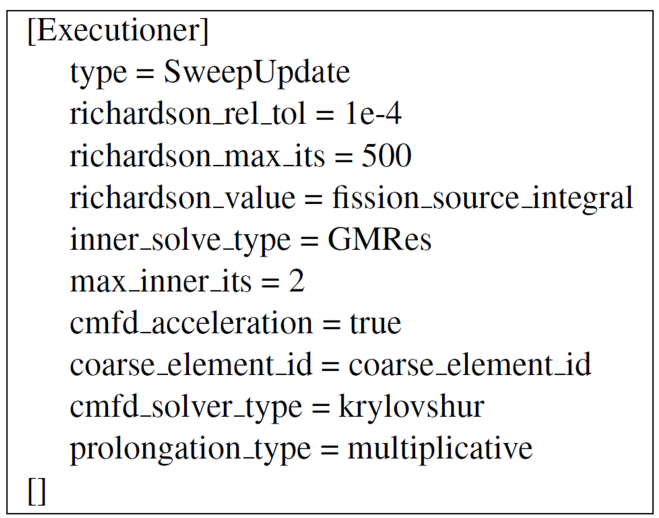

Figure 8: Executioner block for all Griffin calculations.

The 68-group structure was determined to have the smallest eigenvalue errors over a wide range of spectra for a graphite-moderated system, which were used for all test problems discussed in this section. The lethargy widths of the 68-group structure are shown in Fig. 9 together with those of the WIMS-69 group structure [50] for comparison. It has almost uniform lethargy widths above $10 \mathrm{eV}$, and the energy range of the $\mathrm{U}-238$ resonance at $6.67 \mathrm{eV}$ is divided into several groups for the reason explained in Section 4.1 . Note that this group structure is optimized for a fresh uranium fuel in the graphite-moderated system just for this work and will be further optimized for realistic applications to reactor types of interest in future work. The self-shielding treatment is applied to the resonance energy groups ranging from $1.05 \mathrm{eV}$ to $100.52 \mathrm{keV}$, and only the temperature interpolation is involved for the other energy groups. The CSAPI multigroup 
libraries for all problems were generated using the Serpent $2+$ NJOY path explained in Section 2.2. All Serpent2 reference solutions were generated using an explicit particle representation in Serpent2. Serpent2 calculations were performed multiple times using different random particle configurations, based on which averaged results were taken as reference solutions.

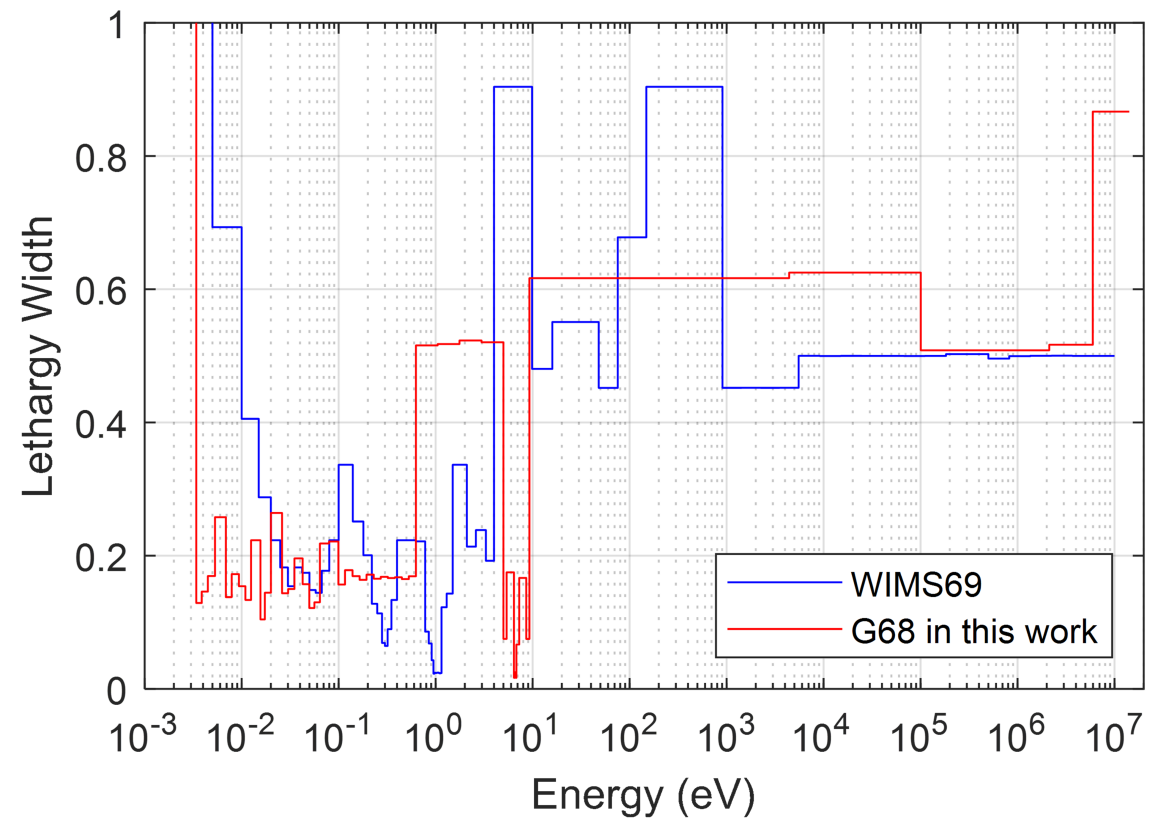

Figure 9: Comparison of lethargy widths of the 68-group structure and the WIMS-69 group structure.

\subsection{VHTR Pin-Cell Problems}

The performance of the Griffin CSAPI was first examined for the prismatic VHTR unit-cell problem, which is shown in Fig. 10 over a wide range of pin-cell design parameters, including double-heterogeneity. A total of 81 problems were constructed by combining different parameters as shown in Table2. The particle radius and packing fraction are fuel particle (microscopic) design parameters, and the compact radius and compact-to-moderator volume ratio are fuel lattice (macroscopic) design parameters. The problems were constructed in a way that, while one parameter varied, the other three parameters were kept the same. For example, to achieve a desired packing fraction with different particle sizes, the number of particles was adjusted. As the number of particles should be an integer and the particle size is fixed, the resulting packing fractions deviate slightly from the targeted packing fraction within the first decimal point. The values of each parameter were chosen to cover most realistic designs. Nine cases of packing fractions and compactto-moderator ratios in Table 3 were chosen to not overlap fuel(kernel)-to-moderator (outer layers of particles 
+ matrix + graphite block) ratios for testing the problems in nine different spectra as shown in Fig. 11 .

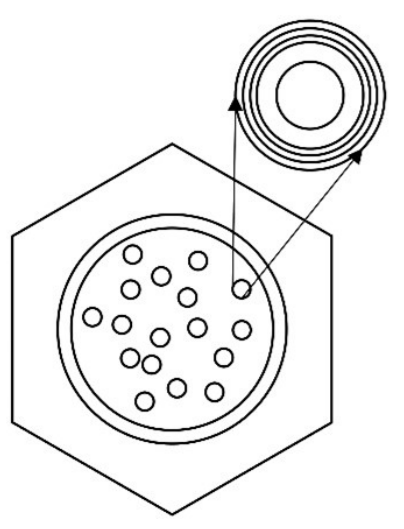

\begin{tabular}{ccccc}
\hline & Region & Radius $(\mathrm{cm})$ & Material & Density $\left(\mathrm{g} / \mathrm{cm}^{3}\right)$ \\
\hline & Fuel Kernel & 0.0175 & 10.36 wt.\% UCO & 10.5 \\
Fuel & Buffer & 0.0275 & Graphite & 1.00 \\
Particle & Inner PyC & 0.0310 & Graphite & 1.90 \\
& SiC & 0.0345 & SiC & 3.20 \\
& Outer PyC & 0.0385 & Graphite & 1.90 \\
\hline \multicolumn{2}{c}{ Fuel Compact } & 0.6225 & Graphite & 1.1995 \\
\hline \multicolumn{2}{c}{ Coolant } & 0.6350 & Helium & 0.0032 \\
\hline \multicolumn{2}{c}{ Graphite Block } & - & Graphite & 1.74 \\
\hline \multicolumn{2}{c}{}
\end{tabular}

Figure 10: Compact design obtained from the NGNP design.

Table 2: Fuel particle and compact parameter values.

\begin{tabular}{|c|c|c|c|}
\hline Scaling Type & Parameter & Abbreviation & Value \\
\hline \multirow{4}{*}{ Microscopic Scaling } & $\mathrm{PS}_{1}$ & 0.0200 \\
\cline { 3 - 4 } & Particle Radius (cm) & $\mathrm{PS}_{2}$ & 0.0385 \\
\cline { 3 - 4 } & & $\mathrm{PS}_{3}$ & 0.0700 \\
\cline { 3 - 4 } & \multirow{3}{*}{ Packing Fraction (\%) } & $\mathrm{PF}_{1}$ & 2.4 \\
\cline { 3 - 4 } & & $\mathrm{PF}_{2}$ & 11.2 \\
\cline { 3 - 4 } Macroscopic Scaling & $\mathrm{PF}_{3}$ & 52.0 \\
\cline { 3 - 4 } & \multirow{3}{*}{ Compact Radius (cm) } & $\mathrm{CS}_{1}$ & 0.4 \\
\cline { 3 - 4 } & & $\mathrm{CS}_{2}$ & 0.8 \\
\cline { 3 - 4 } & \multirow{3}{*}{ Compact-to-Moderator Volume Ratio } & $\mathrm{CS}_{3}$ & 1.2 \\
\cline { 3 - 4 } & & $\mathrm{CM}_{1}$ & 0.606 \\
\cline { 3 - 4 } & $\mathrm{CM}_{2}$ & 1.383 \\
\cline { 3 - 4 } & $\mathrm{CM}_{3}$ & 2.992 \\
\hline
\end{tabular}

For each problem, the homogeneous (single het.) and heterogeneous (double het.) compact configurations were solved. For the double heterogeneous problems, the Serpent 2 results were generated by 25 independent runs with different random particle configurations for each case. Each Serpent 2 simulation was performed with $10^{5}$ particles/cycle, 100 active and 100 inactive cycles. For single heterogeneous problems, $10^{5}$ particles/cycle, 1000 active and 100 inactive cycles were used. The mesh for the VHTR problem of the $\left(\mathrm{CS}_{2}, \mathrm{CM}_{2}\right)$ case in the Griffin calculation is shown in Fig. 12. Different colors mean different cross section zones. Colors other than the red represent the fuel region with six rings of cross section zones with the equal area. Meshes for other problems were constructed in the same manner. A $\mathrm{P}_{2}$ anisotropic scattering order 
Table 3: Fuel to moderator volume ratios.

\begin{tabular}{|c|c|c|c|}
\hline & $\mathrm{CM}_{1}$ & $\mathrm{CM}_{2}$ & $\mathrm{CM}_{3}$ \\
\hline $\mathrm{PF}_{1}$ & $8.55 \mathrm{E}-04$ & $1.32 \mathrm{E}-03$ & $1.70 \mathrm{E}-03$ \\
\hline $\mathrm{PF}_{2}$ & $3.99 \mathrm{E}-03$ & $6.14 \mathrm{E}-03$ & $7.95 \mathrm{E}-03$ \\
\hline $\mathrm{PF}_{3}$ & $1.88 \mathrm{E}-02$ & $2.92 \mathrm{E}-02$ & $3.80 \mathrm{E}-02$ \\
\hline
\end{tabular}

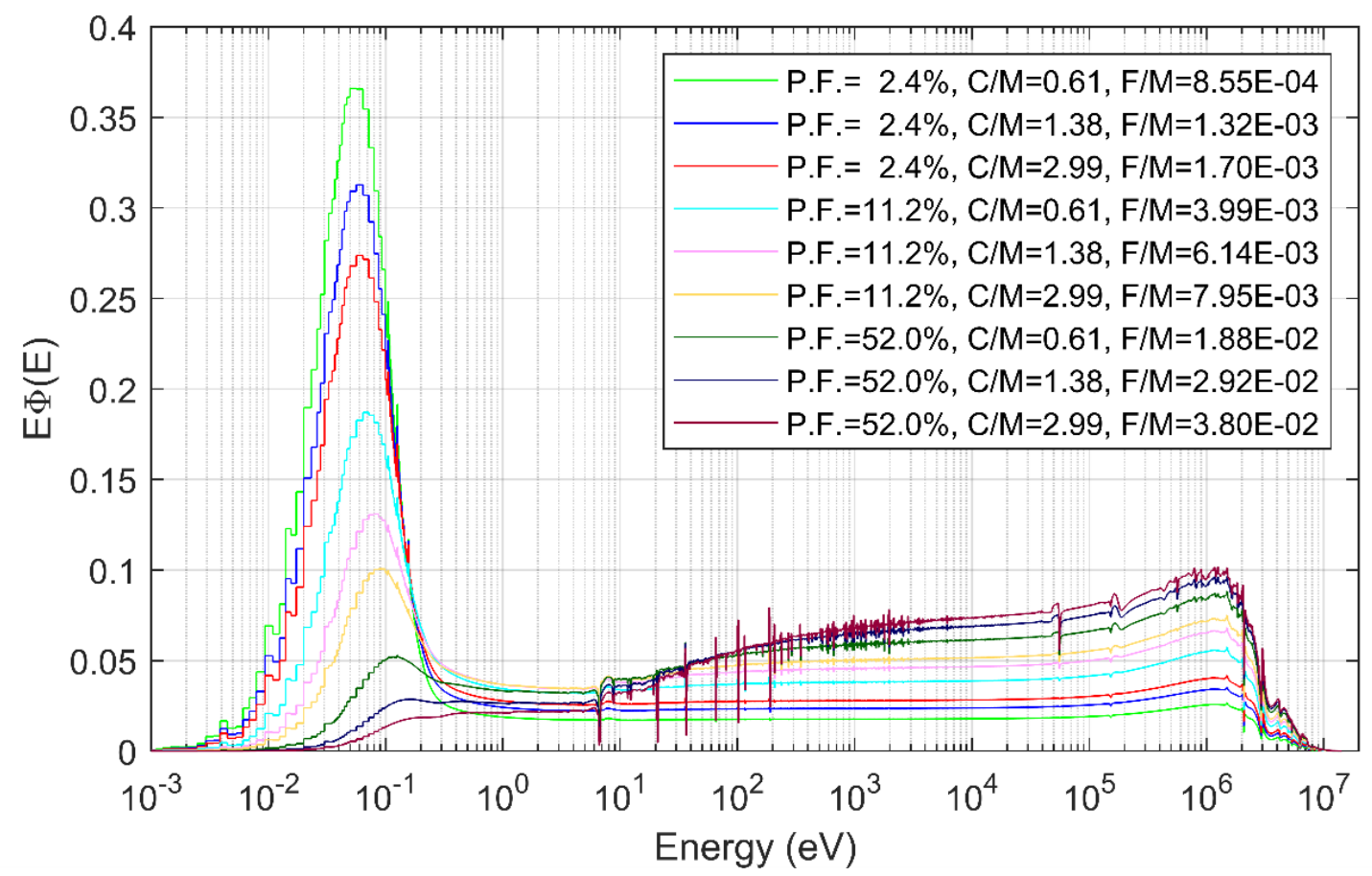

Figure 11: Serpent2 spectra at various fuel-to-moderator volume ratios (at fixed $\mathrm{PS}_{2}$ and $\mathrm{CS}_{2}$ ).

was used.

The CSAPI multigroup library was generated using the pin-cell configuration of the $\left(\mathrm{PF}_{2}, \mathrm{CS}_{2}, \mathrm{CM}_{2}\right)$ problem. That is, the baseline multigroup cross sections were generated using the weighting spectrum of the pink-colored one in Fig. 11, and those in the thermal and fast energy groups are used for all problems with a wide spectrum range in Fig. 11. Multigroup cross sections in the resonance energy range were obtained through the resonance treatment. One thing to note here is that the multigroup scattering kernel generated using the pink-colored spectrum was used for all problems in the current CSAPI scheme. As will be shown in the result, this causes a problem, especially for the under-moderated problems. This will be discussed further in this subsection.

Table 4 shows the Serpent 2 eigenvalue results for the single and double heterogeneous problems in the first and second rows, respectively, and the TRISO heterogeneity effects in the third row for 81 different 


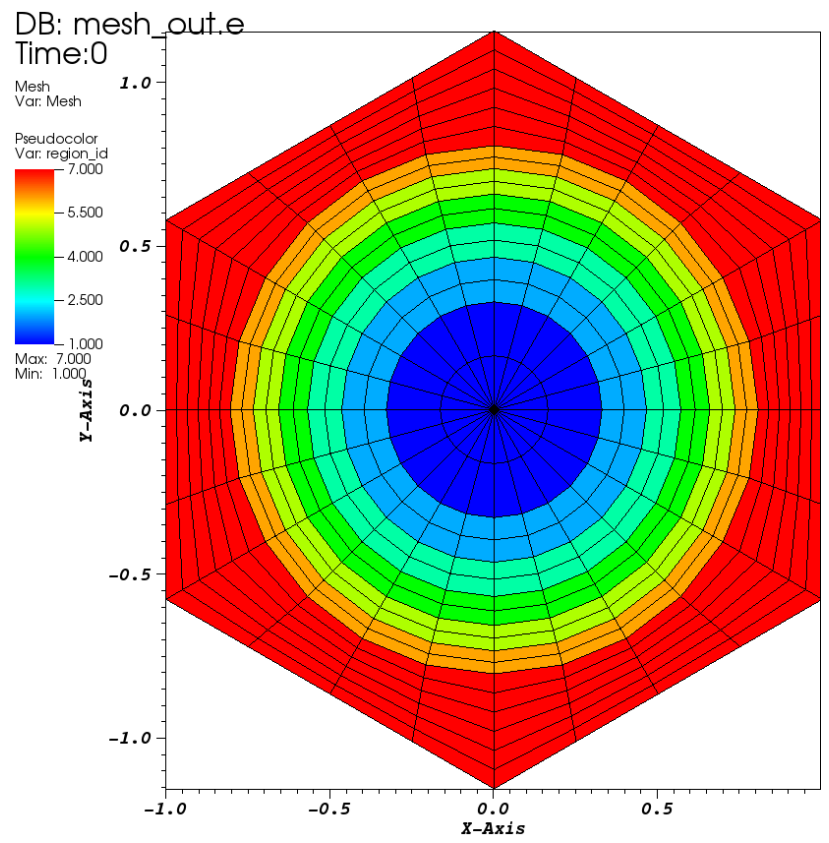

Figure 12: Exodus mesh for the VHTR problem of the $\left(\mathrm{CS}_{2}, \mathrm{CM}_{2}\right)$ case.

pin-cell configurations. The standard deviations of eigenvalues for the single heterogeneous problems range from 0.00002 to 0.00008 , and the average values of 25 standard deviations of eigenvalues for the double heterogeneous problems range from 0.00007 to 0.00024 . The standard deviations of 25 eigenvalues due to the random distribution of particles for the double heterogeneous problems range from $0.00010\left(\mathrm{PS}_{3}, \mathrm{PF}_{1}\right.$, $\left.\mathrm{CS}_{1}, \mathrm{CM}_{1}\right)$ to $0.00069\left(\mathrm{PS}_{3}, \mathrm{PF}_{2}, \mathrm{CS}_{1}, \mathrm{CM}_{2}\right)$, which indicates that a single run of a Monte Carlo code is not sufficient to provide an accurate solution for a stochastic medium even though the random distribution of particles is explicitly used in the run. Even though eigenvalue results of single heterogeneous problems at different particle size cases with other parameters fixed should be identical in principle, they are slightly different as shown in Table 4 since packing fractions vary within the first decimal point for each PF case as explained above.

For the same packing fraction, the reactivity increases with increasing particle size due to the increased spatial self-shielding in particles. Similarly, for the same compact-to-moderator volume ratio, the reactivity increases with increasing compact size due to the increased spatial self-shielding in compacts. When the fuel-to-moderator volume ratio increases, either at the same packing fraction of $\mathrm{PF}_{2}$ or $\mathrm{PF}_{3}\left(\mathrm{CM}_{1}\right.$ to $\left.\mathrm{CM}_{3}\right)$ or at the same compact-to-moderator volume ratio $\left(\mathrm{PF}_{1}\right.$ to $\left.\mathrm{PF}_{3}\right)$, the reactivity decreases due to the reduced neutron moderation even though the relative amount of fuel increases. When the compact-to-moderator volume ratio increases for a very low packing fraction of $\mathrm{PF}_{1}$, the reactivity increases due to the larger effect 
Table 4: Serpent2 eigenvalues of homogeneous (single heterogeneous) and heterogeneous (double heterogeneous) compact problems and TRISO heterogeneity effects in pcm.

\begin{tabular}{|c|c|c|c|c|c|c|c|c|c|c|}
\hline & \multicolumn{3}{|c|}{$\mathrm{CM}_{1}$} & \multicolumn{3}{|c|}{$\mathrm{CM}_{2}$} & \multicolumn{3}{|c|}{$\mathrm{CM}_{3}$} \\
\hline & & $\mathrm{CS}_{1}$ & $\mathrm{CS}_{2}$ & $\mathrm{CS}_{3}$ & $\mathrm{CS}_{1}$ & $\mathrm{CS}_{2}$ & $\mathrm{CS}_{3}$ & $\mathrm{CS}_{1}$ & $\mathrm{CS}_{2}$ & $\mathrm{CS}_{3}$ \\
\hline \multirow{9}{*}{$\mathrm{PF}_{1}$} & & 1.57144 & 1.57383 & 1.57604 & 1.60943 & 1.61145 & 1.61324 & 1.60304 & 1.60417 & 1.60533 \\
\hline & $\mathrm{PS}_{1}$ & 1.60311 & 1.60375 & 1.60430 & 1.65661 & 1.65731 & 1.65795 & 1.65919 & 1.65965 & 1.66008 \\
\hline & & (1257) & (1185) & (1118) & (1769) & (1717) & (1672) & (2111) & (2084) & (2054) \\
\hline & & 1.57213 & 1.57403 & 1.57591 & 1.60952 & 1.61143 & 1.61322 & 1.60280 & 1.60412 & 1.60516 \\
\hline & $\mathrm{PS}_{2}$ & 1.61736 & 1.61670 & 1.61660 & 1.67993 & 1.67997 & 1.68004 & 1.68886 & 1.68909 & 1.68933 \\
\hline & & (1779) & (1677) & (1597) & $(2604)$ & (2532) & $(2465)$ & (3179) & $(3136)$ & (3104) \\
\hline & & 1.56311 & 1.57173 & 1.57592 & 1.60787 & 1.61100 & 1.61326 & 1.60533 & 1.60484 & 1.60528 \\
\hline & $\mathrm{PS}_{3}$ & 1.61469 & 1.62342 & 1.62622 & 1.69612 & 1.70024 & 1.70165 & 1.71820 & 1.71951 & 1.71994 \\
\hline & & (2044) & (2026) & (1963) & $(3236)$ & (3258) & (3220) & (4092) & $(4155)$ & (4153) \\
\hline \multirow{9}{*}{$\mathrm{PF}_{2}$} & & 1.36726 & 1.39791 & 1.42110 & 1.21835 & 1.23313 & 1.24583 & 1.12940 & 1.13518 & 1.14021 \\
\hline & $\mathrm{PS}_{1}$ & 1.40391 & 1.42703 & 1.44574 & 1.25082 & 1.26235 & 1.27285 & 1.15633 & 1.16085 & 1.16518 \\
\hline & & (1910) & $(1460)$ & (1199) & $(2131)$ & (1877) & (1704) & $(2062)$ & (1948) & (1879) \\
\hline & & 1.36747 & 1.39796 & 1.42087 & 1.21863 & 1.23342 & 1.24588 & 1.12946 & 1.13497 & 1.14011 \\
\hline & $\mathrm{PS}_{2}$ & 1.43799 & 1.45521 & 1.47002 & 1.28170 & 1.29049 & 1.29911 & 1.18258 & 1.18595 & 1.18956 \\
\hline & & $(3586)$ & $(2814)$ & (2353) & (4038) & (3586) & (3289) & (3977) & (3788) & (3646) \\
\hline & & 1.36742 & 1.39820 & 1.42074 & 1.21869 & 1.23366 & 1.24567 & 1.12967 & 1.13533 & 1.14002 \\
\hline & $\mathrm{PS}_{3}$ & 1.49348 & 1.50440 & 1.51356 & 1.33581 & 1.34205 & 1.34745 & 1.23079 & 1.23333 & 1.23553 \\
\hline & & (6173) & (5049) & (4317) & (7194) & (6546) & (6064) & (7273) & (6999) & $(6781)$ \\
\hline \multirow{9}{*}{$\mathrm{PF}_{3}$} & & 1.23103 & 1.26680 & 1.29303 & 1.08222 & 1.09794 & 1.11106 & 1.00861 & 1.01409 & 1.01902 \\
\hline & $\mathrm{PS}_{1}$ & 1.25392 & 1.28474 & 1.30853 & 1.10096 & 1.11460 & 1.12647 & 1.02295 & 1.02783 & 1.03229 \\
\hline & & (1483) & (1102) & (916) & (1573) & (1361) & (1231) & (1390) & (1318) & (1261) \\
\hline & & 1.23115 & 1.26676 & 1.29311 & 1.08235 & 1.09799 & 1.11107 & 1.00874 & 1.01413 & 1.01898 \\
\hline & $\mathrm{PS}_{2}$ & 1.27717 & 1.30340 & 1.32453 & 1.11975 & 1.13165 & 1.14234 & 1.03766 & 1.04195 & 1.04601 \\
\hline & & (2927) & (2219) & (1834) & (3086) & (2709) & (2464) & $(2763)$ & (2633) & (2536) \\
\hline & & 1.23057 & 1.26660 & 1.29301 & 1.08183 & 1.09780 & 1.11099 & 1.00821 & 1.01382 & 1.01902 \\
\hline & $\mathrm{PS}_{3}$ & 1.32036 & 1.34046 & 1.35710 & 1.15602 & 1.16598 & 1.17477 & 1.06669 & 1.07065 & 1.07425 \\
\hline & & $(5527)$ & $(4350)$ & $(3652)$ & $(5932)$ & (5326) & (4886) & $(5438)$ & $(5235)$ & $(5045)$ \\
\hline
\end{tabular}

of an increasing relative amount of fuel under sufficient moderation than the effect of decreasing moderation. The TRISO heterogeneity effect tends to be maximized at a proper range of fast-to-thermal flux ratios, and those at the $11.2 \%$ packing fraction are generally larger than those at the other packing fractions as the spectra at the $11.2 \%$ packing fraction have a moderate range of fast-to-thermal flux ratios.

Table 5 shows the eigenvalue errors of the EDC method for single heterogeneous problems. Using the multigroup library generated in the pin-cell configuration of the $\left(\mathrm{PF}_{2}, \mathrm{PS}_{2}, \mathrm{CM}_{2}, \mathrm{CS}_{2}\right)$ case, the results of the EDC method deviate from reference solutions for problems with less moderation than the problem at which the multigroup library was generated (fuel-to-moderator volume ratio higher than 6.14E-3). The highest fuel-to-moderator ratio cases show about $+1 \%$ error in reactivity. This error was not attributed to errors in 
Table 5: Eigenvalue errors (pcm) of the EDC method for single heterogeneous problems.

\begin{tabular}{|c|c|c|c|c|c|c|c|c|c|c|}
\hline \multicolumn{2}{|c|}{} & \multicolumn{3}{c|}{$\mathrm{CM}_{1}$} & \multicolumn{3}{c|}{$\mathrm{CM}_{2}$} & \multicolumn{3}{c|}{$\mathrm{CM}_{3}$} \\
\cline { 3 - 11 } \multicolumn{2}{|c|}{} & $\mathrm{CS}_{1}$ & $\mathrm{CS}_{2}$ & $\mathrm{CS}_{3}$ & $\mathrm{CS}_{1}$ & $\mathrm{CS}_{2}$ & $\mathrm{CS}_{3}$ & $\mathrm{CS}_{1}$ & $\mathrm{CS}_{2}$ & $\mathrm{CS}_{3}$ \\
\hline \multirow{3}{*}{$\mathrm{PF}_{1}$} & $\mathrm{PS}_{1}$ & -8 & -6 & -8 & -11 & -10 & -8 & 4 & -1 & -3 \\
& $\mathrm{PS}_{2}$ & -7 & -10 & -7 & -10 & -9 & -8 & 5 & -1 & 5 \\
& $\mathrm{PS}_{3}$ & -10 & -7 & -8 & -10 & -7 & -9 & 5 & -3 & 1 \\
\hline \multirow{4}{*}{$\mathrm{PF}_{2}$} & $\mathrm{PS}_{1}$ & 10 & -5 & -19 & 67 & 63 & 53 & 196 & 168 & 175 \\
& $\mathrm{PS}_{2}$ & 6 & -4 & -13 & 71 & 62 & 55 & 202 & 171 & 180 \\
& $\mathrm{PS}_{3}$ & 11 & -4 & -18 & 63 & 66 & 59 & 192 & 174 & 175 \\
\hline \multirow{3}{*}{$\mathrm{PF}_{3}$} & $\mathrm{PS}_{1}$ & 239 & 166 & 162 & 724 & 653 & 624 & 1132 & 1045 & 1047 \\
& $\mathrm{PS}_{2}$ & 238 & 168 & 157 & 724 & 644 & 621 & 1126 & 1041 & 1050 \\
& $\mathrm{PS}_{3}$ & 234 & 171 & 164 & 720 & 656 & 631 & 1134 & 1064 & 1048 \\
\hline
\end{tabular}

effective cross sections evaluated from the EDC method, but to an overestimated multigroup down-scattering kernel of graphite from the epithermal energy range to the thermal energy range. That is, over-moderation led to a higher reactivity. Since erroneous cases are under-moderated ones, reactivity would be very sensitive to errors in the amount of moderation.

To be more specific, the reason for the overestimation of the multigroup down-scattering kernel can be explained from Fig. 13 , which shows the spectrum of the $\left(\mathrm{PF}_{3}, \mathrm{CM}_{3}\right)$ case and the spectrum used for generating the multigroup library. As noted in Section 2.3.4, it is often that a multigroup scattering kernel does not account for a problem-dependent spectrum, as illustrated in this example. For simplicity, the multigroup quantities for an s-wave scattering kernel are expressed as

$$
\begin{aligned}
& \sigma_{g \rightarrow g}=\int_{E_{g}}^{E_{g-1}} \frac{\sigma_{s}(E)}{1-\alpha}\left(1-\frac{E_{g}}{E}\right) w(E) d E, \\
& \sigma_{g \rightarrow g^{\prime}}=\int_{E_{g}}^{E_{g-1}} \frac{\sigma_{s}(E)}{1-\alpha} \frac{\Delta E_{g^{\prime}}}{E} w(E) d E \text { for } g \neq g^{\prime},
\end{aligned}
$$

where $E_{g}$ and $E_{g-1}$ are the lower and upper boundaries of energy group $g$, respectively, and $w(E)$ is a weighting function. Since $\sigma_{s}(E)$ is nearly constant for most major moderators, its accuracy mainly depends on the overall slope of the within-group spectrum. Using the black instead of the red spectrum in Fig. 13 results in an overestimated down-scattering kernel and an underestimated self-scattering kernel due to the higher weighting on the lower energy part within the group. Errors in self- and down-scattering kernels from energy groups G1-G4 in Fig. 13 are shown in Table 6. Down-scattering cross sections of graphite can easily produce errors up to about 7\%. Its consequence on the spectrum result would be huge for the undermoderated system, as illustrated in Fig. 14, which shows the spectrum results for the $\left(\mathrm{PF}_{3}, \mathrm{CM}_{3}\right)$ case. Due 
to an overestimated multigroup down-scattering kernel and an underestimated multigroup self-scattering kernel shown in Table 6, the spectrum in the thermal and epithermal energy range has about $+2.5 \%$ and -2.5 to $-5 \%$ errors, respectively, resulting in a reactivity error of about $+1 \%$.

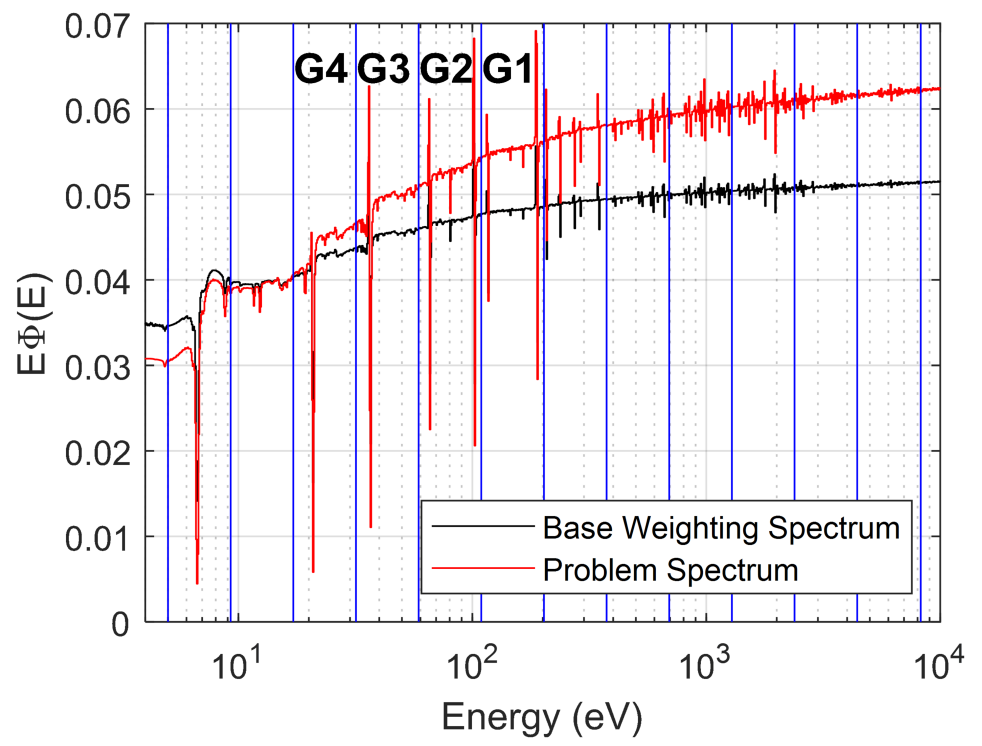

Figure 13: Comparison of base weighting spectrum used for the multigroup library generation and problem spectrum in the epithermal energy range.

Table 6: Errors of self- and down-scattering kernels of graphite from Group-1 to Group-4 in Fig. 13 due to the use of spectrum with overestimated moderation.

\begin{tabular}{|c|c|c|c|c|}
\hline & G1 & G2 & G3 & G4 \\
\hline Self & $-0.57 \%$ & $-0.81 \%$ & $-1.83 \%$ & $-2.14 \%$ \\
\hline Down & $+1.71 \%$ & $+2.44 \%$ & $+5.91 \%$ & $+7.10 \%$ \\
\hline
\end{tabular}

To improve the results, multigroup scattering kernels for nine representative spectra of different fuel-tomoderator ratios were generated using NJOY with corresponding spectra, producing nine different multigroup libraries. Note that the only differences among the libraries are the multigroup scattering kernel. Table 7 shows the improved results. Errors of about $1 \%$ were reduced to less than $200 \mathrm{pcm}$, and all results agreed well with the Serpent 2 results. Table 8 and Table 9 show errors in the eigenvalues and the TRISO heterogeneity effect of double heterogeneous problems solved using the nine CSAPI multigroup libraries and the EDC + ILSS methods. All results agreed very well without any noticeable dependency on double heterogeneity parameters. This indicates the strong need for introducing a parameter accounting for this problem-dependent spectrum in the multigroup scattering kernel for major moderators in the CSAPI 

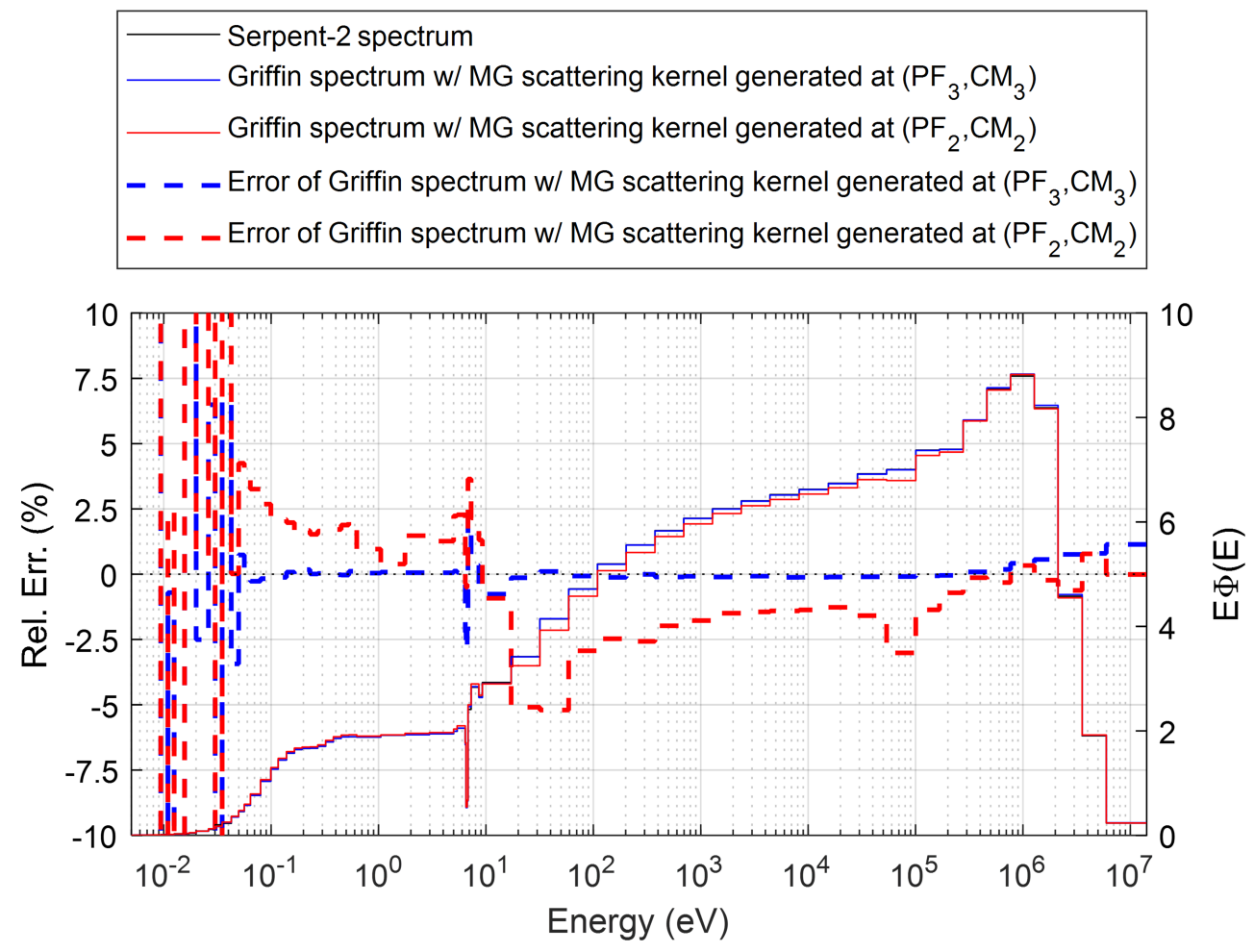

Figure 14: Comparison of spectrum for the $\left(\mathrm{PF}_{3}, \mathrm{CM}_{3}\right)$ case obtained with the multigroup scattering kernel of $\left(\mathrm{PF}_{2}, \mathrm{CM}_{2}\right)$ and $\left(\mathrm{PF}_{3}, \mathrm{CM}_{3}\right)$.

multigroup library. 
Table 7: Eigenvalue errors ( $\mathrm{pcm}$ ) of the EDC method for single heterogeneous problems with update of multigroup scattering kernels.

\begin{tabular}{|c|c|c|c|c|c|c|c|c|c|c|}
\hline \multicolumn{2}{|c|}{} & \multicolumn{3}{c|}{$\mathrm{CM}_{1}$} & \multicolumn{3}{c|}{$\mathrm{CM}_{2}$} & \multicolumn{3}{c|}{$\mathrm{CM}_{3}$} \\
\cline { 3 - 11 } \multicolumn{2}{|c|}{} & $\mathrm{CS}_{1}$ & $\mathrm{CS}_{2}$ & $\mathrm{CS}_{3}$ & $\mathrm{CS}_{1}$ & $\mathrm{CS}_{2}$ & $\mathrm{CS}_{3}$ & $\mathrm{CS}_{1}$ & $\mathrm{CS}_{2}$ & $\mathrm{CS}_{3}$ \\
\hline \multirow{3}{*}{$\mathrm{PF}_{1}$} & $\mathrm{PS}_{1}$ & 36 & 36 & 35 & 43 & 43 & 45 & 62 & 56 & 55 \\
& $\mathrm{PS}_{2}$ & 38 & 33 & 36 & 43 & 45 & 45 & 63 & 57 & 62 \\
& $\mathrm{PS}_{3}$ & 33 & 36 & 35 & 43 & 46 & 44 & 61 & 54 & 58 \\
\hline \multirow{4}{*}{$\mathrm{PF}_{2}$} & $\mathrm{PS}_{1}$ & 59 & 44 & 28 & 68 & 66 & 57 & 130 & 102 & 110 \\
& $\mathrm{PS}_{2}$ & 56 & 44 & 35 & 71 & 65 & 59 & 134 & 106 & 115 \\
& $\mathrm{PS}_{3}$ & 60 & 45 & 29 & 64 & 68 & 63 & 125 & 108 & 110 \\
\hline \multirow{3}{*}{$\mathrm{PF}_{3}$} & $\mathrm{PS}_{1}$ & -1 & -49 & -36 & 78 & 27 & 18 & 182 & 105 & 117 \\
& $\mathrm{PS}_{2}$ & -3 & -47 & -41 & 75 & 23 & 17 & 176 & 101 & 121 \\
& $\mathrm{PS}_{3}$ & -6 & -43 & -33 & 71 & 30 & 25 & 185 & 123 & 119 \\
\hline
\end{tabular}

Table 8: Eigenvalue errors (pcm) of the EDC + ILSS method for double heterogeneous problems with update of multigroup scattering kernels.

\begin{tabular}{|c|c|c|c|c|c|c|c|c|c|c|}
\hline \multicolumn{2}{|c|}{} & \multicolumn{3}{c|}{$\mathrm{CM}_{1}$} & \multicolumn{3}{c|}{$\mathrm{CM}_{2}$} & \multicolumn{3}{c|}{$\mathrm{CM}_{3}$} \\
\cline { 3 - 11 } \multicolumn{2}{|c|}{} & $\mathrm{CS}_{1}$ & $\mathrm{CS}_{2}$ & $\mathrm{CS}_{3}$ & $\mathrm{CS}_{1}$ & $\mathrm{CS}_{2}$ & $\mathrm{CS}_{3}$ & $\mathrm{CS}_{1}$ & $\mathrm{CS}_{2}$ & $\mathrm{CS}_{3}$ \\
\hline \multirow{3}{*}{$\mathrm{PF}_{1}$} & $\mathrm{PS}_{1}$ & 24 & 23 & 24 & 30 & 30 & 30 & 39 & 37 & 38 \\
& $\mathrm{PS}_{2}$ & 15 & 16 & 16 & 17 & 17 & 22 & 22 & 23 & 22 \\
& $\mathrm{PS}_{3}$ & 18 & 19 & 16 & 15 & 17 & 15 & 16 & 15 & 14 \\
\hline \multirow{4}{*}{$\mathrm{PF}_{2}$} & $\mathrm{PS}_{1}$ & 44 & 48 & 48 & 43 & 59 & 59 & 94 & 83 & 93 \\
& $\mathrm{PS}_{2}$ & 20 & 30 & 29 & 23 & 34 & 33 & 52 & 51 & 51 \\
& $\mathrm{PS}_{3}$ & 9 & 13 & 20 & 3 & 16 & 14 & 16 & 19 & 26 \\
\hline \multirow{3}{*}{$\mathrm{PF}_{3}$} & $\mathrm{PS}_{1}$ & 17 & 11 & -5 & 43 & 46 & 36 & 165 & 113 & 129 \\
& $\mathrm{PS}_{2}$ & -3 & 14 & 12 & 4 & 26 & 28 & 123 & 83 & 106 \\
& $\mathrm{PS}_{3}$ & -10 & 8 & 14 & -28 & -5 & -8 & 83 & 58 & 74 \\
\hline
\end{tabular}

Table 9: Errors (pcm) in TRISO heterogeneity effect of the ILSS method (Table 8- Table 7).

\begin{tabular}{|l|l|c|c|c|c|c|c|c|c|c|}
\hline \multicolumn{2}{|c|}{} & \multicolumn{3}{c|}{$\mathrm{CM}_{1}$} & \multicolumn{3}{c|}{$\mathrm{CM}_{2}$} & \multicolumn{3}{c|}{$\mathrm{CM}_{3}$} \\
\cline { 3 - 11 } \multicolumn{2}{|c|}{} & $\mathrm{CS}_{1}$ & $\mathrm{CS}_{2}$ & $\mathrm{CS}_{3}$ & $\mathrm{CS}_{1}$ & $\mathrm{CS}_{2}$ & $\mathrm{CS}_{3}$ & $\mathrm{CS}_{1}$ & $\mathrm{CS}_{2}$ & $\mathrm{CS}_{3}$ \\
\hline \multirow{3}{*}{$\mathrm{PF}_{1}$} & $\mathrm{PS}_{1}$ & -12 & -14 & -11 & -13 & -13 & -15 & -23 & -19 & -17 \\
& $\mathrm{PS}_{2}$ & -22 & -17 & -19 & -26 & -27 & -23 & -40 & -34 & -40 \\
& $\mathrm{PS}_{3}$ & -15 & -17 & -19 & -28 & -29 & -29 & -45 & -39 & -44 \\
\hline \multirow{4}{*}{$\mathrm{PF}_{2}$} & $\mathrm{PS}_{1}$ & -16 & 4 & 20 & -25 & -7 & 3 & -35 & -19 & -17 \\
& $\mathrm{PS}_{2}$ & -36 & -14 & -6 & -49 & -30 & -26 & -83 & -56 & -64 \\
& $\mathrm{PS}_{3}$ & -51 & -32 & -9 & -61 & -53 & -49 & -109 & -89 & -85 \\
\hline \multirow{3}{*}{$\mathrm{PF}_{3}$} & $\mathrm{PS}_{1}$ & 18 & 60 & 32 & -35 & 19 & 17 & -17 & 8 & 13 \\
& $\mathrm{PS}_{2}$ & -1 & 61 & 53 & -71 & 3 & 11 & -53 & -18 & -15 \\
& $\mathrm{PS}_{3}$ & -4 & 51 & 47 & -98 & -35 & -33 & -102 & -65 & -45 \\
\hline
\end{tabular}


Fig. 15 shows the relative errors of compact-averaged multigroup U-238 capture cross sections for all $\mathrm{CS}$ and $\mathrm{CM}$ cases of $\mathrm{PF}_{2}$ and $\mathrm{PF}_{3}$ cases at $\mathrm{PS}_{2}$. In the fast and thermal energy range, cross sections match well among all cases. In the resonance energy groups at major U-238 resonances, cross section errors are all within $\pm 0.5 \%$ for both the single and double heterogeneous problems. About $+4.5 \%$ error in the energy range of 3-5 eV resulted from the neglect of up-scattering in the slowing down problem. This is illustrated in Fig. 16, which compares the Serpent2 spectrum and the slowing down spectrum solution of Griffin for the single energy group from 3 to $5 \mathrm{eV}$ in the left figure and for an energy range of 5 to $16.5 \mathrm{eV}$ in the right figure. As seen in the left figure, the higher weighting on the energy portion where cross sections are higher for the red line results in the higher multigroup cross section in that energy group. The spectrum difference caused by neglecting up-scattering is clearly seen in the right figure. Since Serpent 2 considers thermal up-scattering up to $400 \mathrm{k}_{B} \mathrm{~T}$, which is around $10 \mathrm{eV}$ for the room temperature, the blue and red curves deviate starting from $10 \mathrm{eV}$. This difference motivated us to divide the energy range near the $6.67 \mathrm{eV}$ resonance of U-238 into several groups. An effective cross section in a single group from $5 \mathrm{eV}$ to $9 \mathrm{eV}$, as in the WIMS-69 group structure, has 3-4\% error, which results in more than a -100 pcm bias on reactivity. This indicates the strong need for accounting for thermal up-scattering in the slowing down calculation. In addition, the resonance up-scattering from heavy isotopes needs to be considered in the slowing down calculation as well. These are particularly important for high temperature problems with graphite moderator, which will be investigated in future work. 

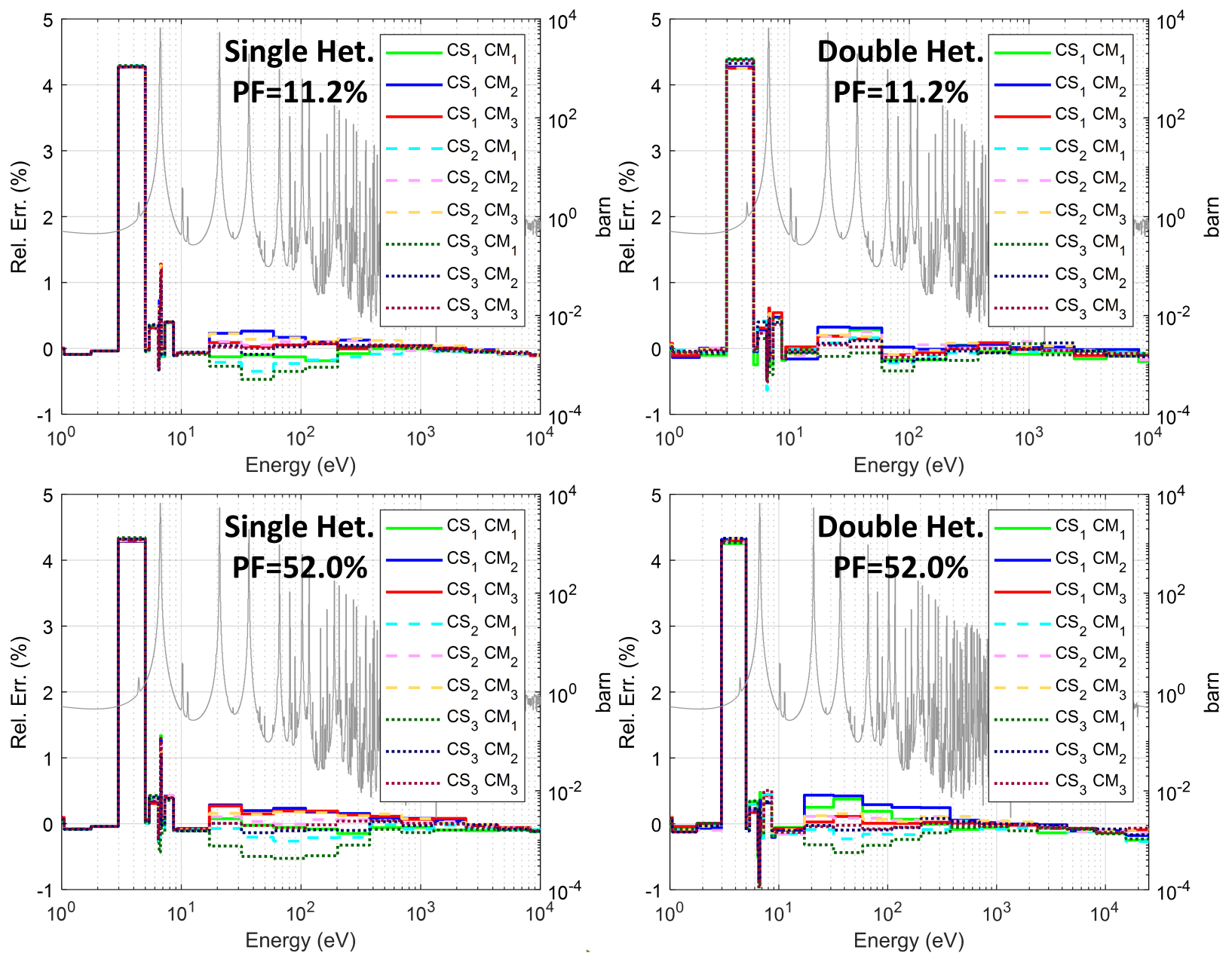

Figure 15: Relative errors of compact-averaged U-238 multigroup capture cross sections for all CS and $\mathrm{CM}$ cases of $\mathrm{PF}_{2}$ and $\mathrm{PF}_{3}$ cases at $\mathrm{PS}_{2}$.
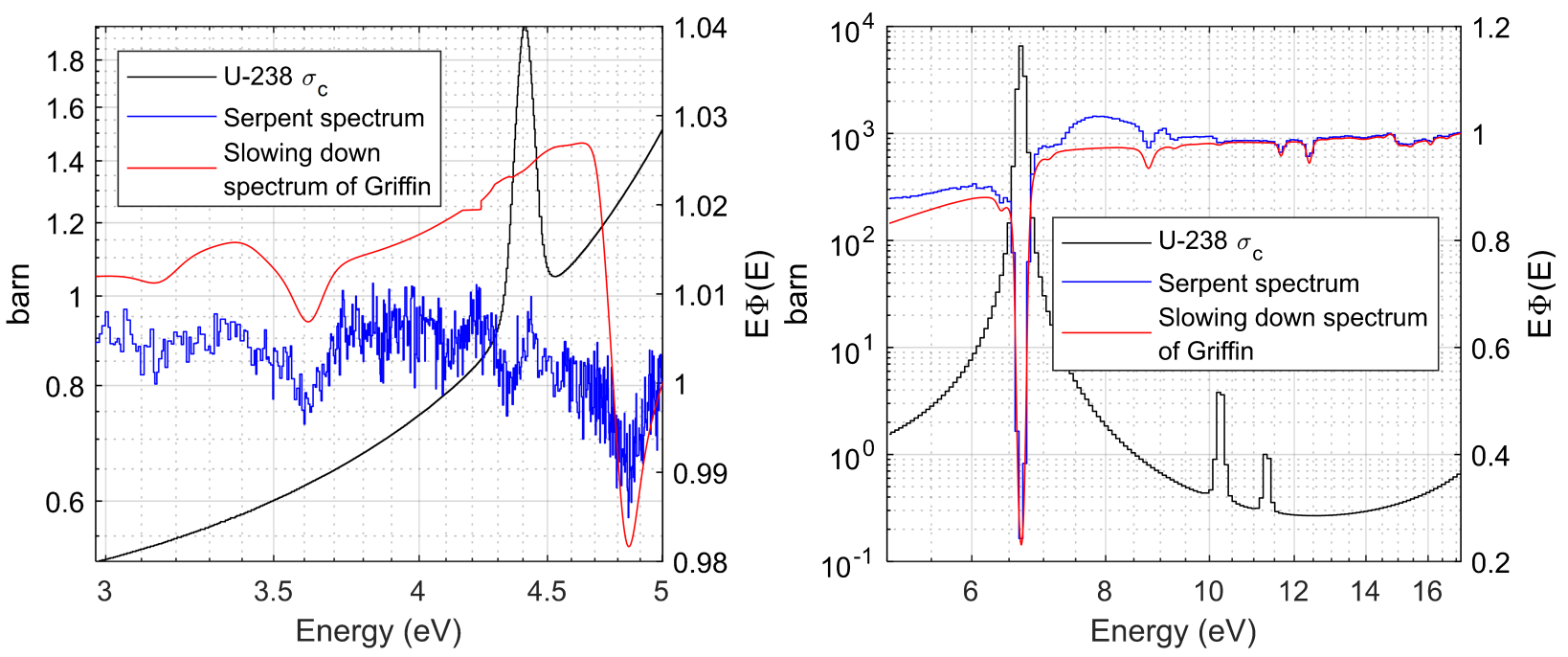

Figure 16: Comparison of Serpent2 spectrum and slowing down spectrum solution of Griffin at $6.67 \mathrm{eV}$ U-238 resonance. 


\subsection{EMPIRE Unit-Cell Problem}

The Griffin CSAPI was also verified for the microreactor unit-cell problem inspired from the EMPIRE benchmark concept initially developed at Los Alamos National Laboratory (LANL) [69]. The unit-cell problem solved in this work is shown in Fig. 17, which was imported from a modeling exercise for a microreactor concept in Reference [70]. For simplicity, the problem in Reference [70] was modified to a 2D problem. A traditional TRISO particle with $20.15 \mathrm{wt} . \%$ UCO fuel was adopted in a hexagonal graphite matrix with a packing fraction of $37.5 \%$. Room temperature was assumed.

For the double heterogeneous problem, Serpent 2 results were generated by 15 independent runs with different random particle configurations for each case. For each run, the same random particle configuration was used in all fuel compacts. Each Serpent 2 simulation was performed with $10^{5}$ particles/cycle, 100 active and 100 inactive cycles. For the single heterogeneous problem, $10^{5}$ particles/cycle, 1000 active and 100 inactive cycles were used. For Griffin calculations, an Exodus mesh shown in Fig. 17 was used. A $\mathrm{P}_{3}$ anisotropic scattering order was used, as this problem has a strong anisotropic scattering effect due to the large amount of light- and intermediate-mass nuclides. One cross section zone was used for each material region.

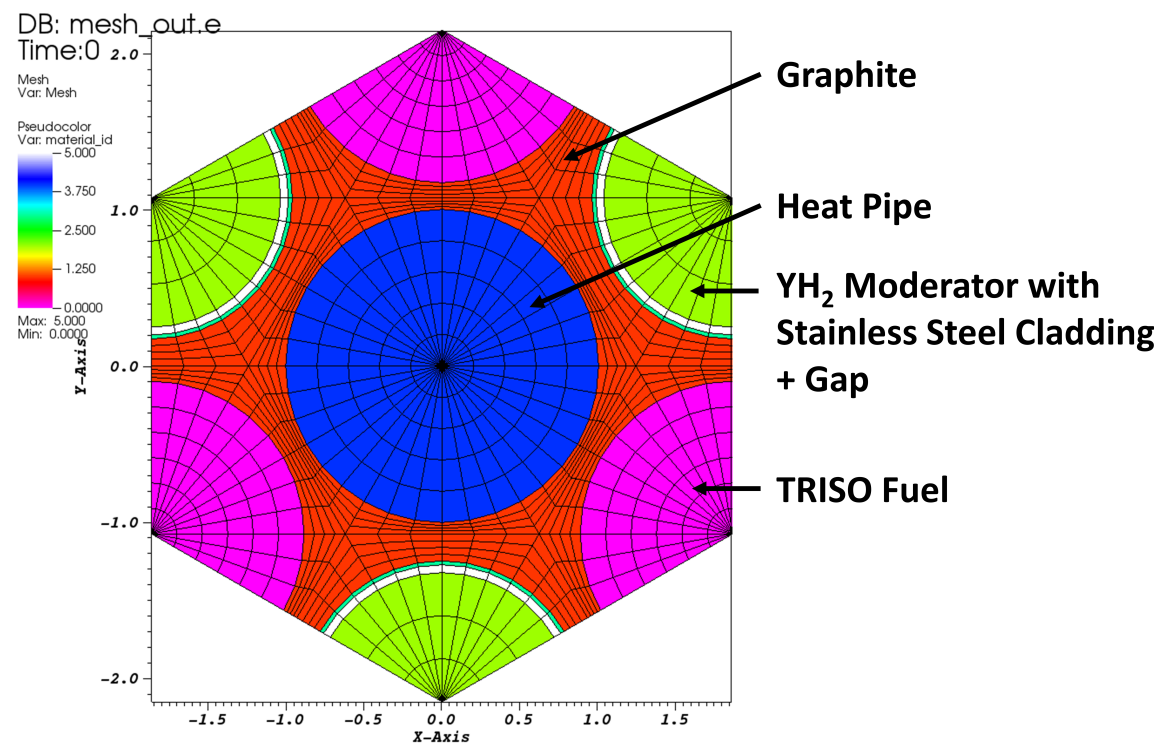

Figure 17: Exodus mesh and material layout of the EMPIRE 2D unit-cell problem.

The EDC radius of the fuel compact was $1.787 \mathrm{~cm}$, which is much larger than $1.129 \mathrm{~cm}$, the size determined from the volume preservation of the fuel cell only. This is because only the fuel is assumed to 
be black and other materials serve as potential scattering sources. The size of the EDC is determined by calculating the amount of graphite that produces the equivalent neutron source to the fuel region to which all the other materials in the actual lattice would contribute.

Table 10 shows the eigenvalue results of Serpent 2 and Griffin. Two uncertainties of the Serpent 2 eigenvalue for the double heterogeneous problem are the average of 15 eigenvalue standard deviations and the standard deviation of 15 eigenvalues, respectively. One thing to note is that the TRISO heterogeneity effect is negative, which is opposite to the case observed from the VHTR problems. This negative TRISO heterogeneity effect is caused by the fact that the thermal utilization factor of the double heterogeneous problem is smaller than that of the single heterogeneous problem. Table 11 shows two group reaction rates per fission neutron at each region with the energy boundary of $5.0 \mathrm{eV}$. In the " $\Delta$ het." row, it is shown that there are fewer captures (-6.686E-3) in the resonance energy range of the fuel due to spatial self-shielding and more thermal captures from $\mathrm{YH}_{2}(+4.690 \mathrm{E}-3)$, heat pipe (+7.838E-3), and stainless-steel cladding (+1.316E-3). These reaction rate differences are translated to the reactivity difference of $-757 \mathrm{pcm}$, which is consistent with the TRISO heterogeneity effect of $-767 \mathrm{pcm}$ in Table 10 . This occurs because, by using TRISO particles, fuel becomes more transparent to neutrons due to less capture in the resonance energy range, more downscattering from the moderator, and more thermal captures in $\mathrm{YH}_{2}$, heat pipe, and stainless-steel cladding take place. Note that the thermal capture cross sections of those three regions are larger than that of fuel due to the low density of fuel $(2.247 \mathrm{~g} / \mathrm{cc})$.

Table 10: Eigenvalue results of Griffin compared to Serpent2 for EMPIRE unit-cell problem.

\begin{tabular}{|c|c|c|c|c|c|c|c|}
\hline \multirow{2}{*}{\multicolumn{2}{|c|}{ Code }} & \multicolumn{2}{|c|}{ Single Het. } & \multicolumn{2}{|c|}{ Double Het. } & \multirow{2}{*}{$\Delta$ Het. (pcm) } & \multirow{2}{*}{$\Delta \Delta$ Het. $(\mathrm{pcm})$} \\
\hline & & K-eff & $\Delta \rho(\mathrm{pcm})$ & K-eff & $\Delta \rho(\mathrm{pcm})$ & & \\
\hline \multicolumn{2}{|c|}{ Serpent2 } & 0.93845 & \pm 5 & 0.93174 & $\pm 14^{\mathrm{a}} / \pm 33^{\mathrm{b}}$ & -767 & - \\
\hline Griffin & EDC & 0.93919 & +84 & 0.93325 & +173 & -678 & +89 \\
\hline
\end{tabular}

a: Average of 15 eigenvalue standard deviations, b: Standard deviation of 15 eigenvalues.

Table 12 shows the absolute errors of 2-group reaction rates per fission neutron obtained using the EDC + ILSS method. Reactivity errors of $+39,+32$ and $+16 \mathrm{pcm}$ are contributed from the fuel, $\mathrm{YH}_{2}$ and stainless-steel cladding, respectively, for the single heterogeneous problem, and those errors of $+73,+53$, +26 , and $+21 \mathrm{pcm}$ are contributed from the fuel, $\mathrm{YH}_{2}$, heat pipe and stainless-steel cladding, respectively, for the double heterogeneous problem. Note that, unlike in the VHTR pin-cell problem, a small error of the resonance capture rate in the fuel region can cause non-negligible sequential errors in non-fuel regions due 
Table 11: Two-group reaction rates per fission neutron of Serpent2 for each region.

\begin{tabular}{|c|c|c|c|c|c|c|c|}
\hline \multirow{2}{*}{ Prob. } & \multirow{2}{*}{ Energy Group } & \multicolumn{5}{|c|}{ Capture } & Fission \\
\hline & & Fuel & $\mathrm{YH}_{2}$ & Heat Pipe & Graphite & S.S. Clad & Fuel \\
\hline \multirow{3}{*}{ Single Het. } & Fast & $8.914 \mathrm{E}-2$ & $9.038 \mathrm{E}-3$ & $2.532 \mathrm{E}-2$ & 4.929E-4 & $4.160 \mathrm{E}-3$ & $4.304 \mathrm{E}-2$ \\
\hline & Thermal & $7.504 \mathrm{E}-2$ & $1.655 \mathrm{E}-1$ & $2.428 \mathrm{E}-1$ & $1.568 \mathrm{E}-3$ & 4.253E-2 & $3.670 \mathrm{E}-1$ \\
\hline & Sum & $1.6 \overline{4} 2 \bar{E}-1$ & $\overline{1} . \overline{7} \overline{4} 6 \overline{\mathrm{E}}-1$ & $2 . \overline{68} \overline{1} \overline{\mathrm{E}}-\overline{1}$ & $\overline{2.0} \overline{6} 0 \overline{\mathrm{E}}-3$ & $\overline{4} . \overline{6} 6 \overline{9} \overline{\mathrm{E}}-\overline{2}$ & $4.100 \overline{\mathrm{E}}-1$ \\
\hline \multirow{3}{*}{ Double Het. } & Fast & $8.245 \mathrm{E}-2$ & $9.136 \mathrm{E}-3$ & $2.559 \mathrm{E}-2$ & $4.951 \mathrm{E}-4$ & $4.203 \mathrm{E}-3$ & $4.324 \mathrm{E}-2$ \\
\hline & Thermal & $7.511 \mathrm{E}-2$ & $1.702 \mathrm{E}-1$ & $2.506 \mathrm{E}-1$ & $1.619 \mathrm{E}-3$ & 4.384E-2 & $3.667 \mathrm{E}-1$ \\
\hline & $\mathrm{Sum}^{-}$ & $-\overline{1.5 \overline{7} \mathrm{E}-1}$ & $\overline{1} . \overline{7} \overline{4} \overline{\mathrm{E}}-\overline{1}$ & $-\overline{2} \cdot \overline{6} \overline{2} \overline{\mathrm{E}}-\overline{1}$ & $2.1 \overline{14 \mathrm{E}-3}$ & $\overline{4} . \overline{8} 0 \overline{5} \overline{\mathrm{E}}-\overline{2}$ & $4.100 \mathrm{E}-1$ \\
\hline \multirow{3}{*}{$\Delta$ Het. } & Fast & $-6.686 \mathrm{E}-3$ & $9.835 \mathrm{E}-5$ & $2.704 \mathrm{E}-4$ & $2.252 \mathrm{E}-6$ & $4.278 \mathrm{E}-5$ & $1.941 \mathrm{E}-4$ \\
\hline & Thermal & $6.811 \mathrm{E}-5$ & $4.690 \mathrm{E}-3$ & $7.838 \mathrm{E}-3$ & $5.160 \mathrm{E}-5$ & $1.316 \mathrm{E}-3$ & $-2.182 \mathrm{E}-4$ \\
\hline & Sum & $-\overline{6} \cdot \overline{6} \overline{8} \overline{\mathrm{E}}-\overline{3}$ & $\overline{4} . \overline{7} 8 \overline{8} \overline{\mathrm{E}}-\overline{3}$ & $-\overline{8.10} \overline{8} \overline{\mathrm{E}}-\overline{3}$ & $\overline{5} . \overline{8} 85 \mathrm{E}-5$ & $1 . \overline{3} 5 \overline{9} \overline{\mathrm{E}}-\overline{3}$ & $-\overline{-2 . \overline{40}} \overline{\mathrm{B}} \overline{\mathrm{E}}-\overline{5}$ \\
\hline
\end{tabular}

to a large streaming effect, which makes the problem more challenging to solve.

Table 12: Absolute errors of 2-group reaction rates per fission neutron obtained by the EDC + ILSS method of Griffin for each region.

\begin{tabular}{|c|c|c|c|c|c|c|c|}
\hline \multirow{2}{*}{ Prob. } & \multirow{2}{*}{ Energy Group } & \multicolumn{5}{|c|}{ Capture } & Fission \\
\hline & & Fuel & $\mathrm{YH}_{2}$ & Heat Pipe & Graphite & S.S. Clad & Fuel \\
\hline \multirow{3}{*}{ Single Het. } & Fast & $-1.491 \mathrm{E}-3$ & $-4.206 \mathrm{E}-4$ & $-8.786 \mathrm{E}-4$ & $1.561 \mathrm{E}-6$ & $-2.195 \mathrm{E}-4$ & $-2.178 \mathrm{E}-4$ \\
\hline & Thermal & $1.099 \mathrm{E}-3$ & $9.585 \mathrm{E}-5$ & $9.206 \mathrm{E}-4$ & $4.489 \mathrm{E}-6$ & $6.381 \mathrm{E}-5$ & $2.172 \mathrm{E}-4$ \\
\hline & Sum & $-3.9 \overline{2} \overline{\mathrm{E}}-\overline{4}$ & $-3.2 \overline{4} \overline{\mathrm{E}}-\overline{4}$ & $\overline{4} . \overline{205} \overline{\mathrm{E}}-\overline{5}$ & $\overline{6} . \overline{0} 5 \overline{1} \overline{\mathrm{E}}-\overline{6}$ & $-\overline{1.55} \overline{7} \overline{\mathrm{E}}-\overline{4}$ & $-\overline{6} .09 \overline{4} \bar{E}-\overline{7}$ \\
\hline \multirow{3}{*}{ Double Het. } & Fast & $-1.871 \mathrm{E}-3$ & $-4.412 \mathrm{E}-4$ & $-9.099 \mathrm{E}-4$ & $2.186 \mathrm{E}-6$ & $-2.253 \mathrm{E}-4$ & $-3.645 \mathrm{E}-4$ \\
\hline & Thermal & $1.146 \mathrm{E}-3$ & $-8.720 \mathrm{E}-5$ & $6.517 \mathrm{E}-4$ & $2.095 \mathrm{E}-6$ & $1.700 \mathrm{E}-5$ & $3.701 \mathrm{E}-4$ \\
\hline & Sum & $-\overline{7} . \overline{25} \overline{2} \overline{\mathrm{E}}-\overline{4}$ & $-5.2 \overline{8} \overline{4} \bar{E}-\overline{4}$ & $-2.5 \overline{8} 2 \overline{\mathrm{E}}-\overline{4}$ & $\overline{4} . \overline{2} 8 \overline{2} \overline{\mathrm{E}}-\overline{6}$ & $-\overline{-2} . \overline{0} 8 \overline{3} \overline{\mathrm{E}}-\overline{4}$ & $\overline{5} . \overline{6} \overline{5 \mathrm{E}}-\overline{6}$ \\
\hline
\end{tabular}

Fig. 18 and 19 show relative errors of spectrum and compact-averaged multigroup capture cross sections of Griffin for the fuel compact. Notable spectrum errors are observed in the high energy range above $1 \mathrm{MeV}$ and the energy groups around $1 \mathrm{eV}$. Multigroup cross sections agree well within 2\% relative errors, but the consistent underestimation of capture cross sections in the major U-238 resonance groups is one of the main contributors for the slightly overestimated reactivity error. The causes of these errors need to be investigated in future work. 


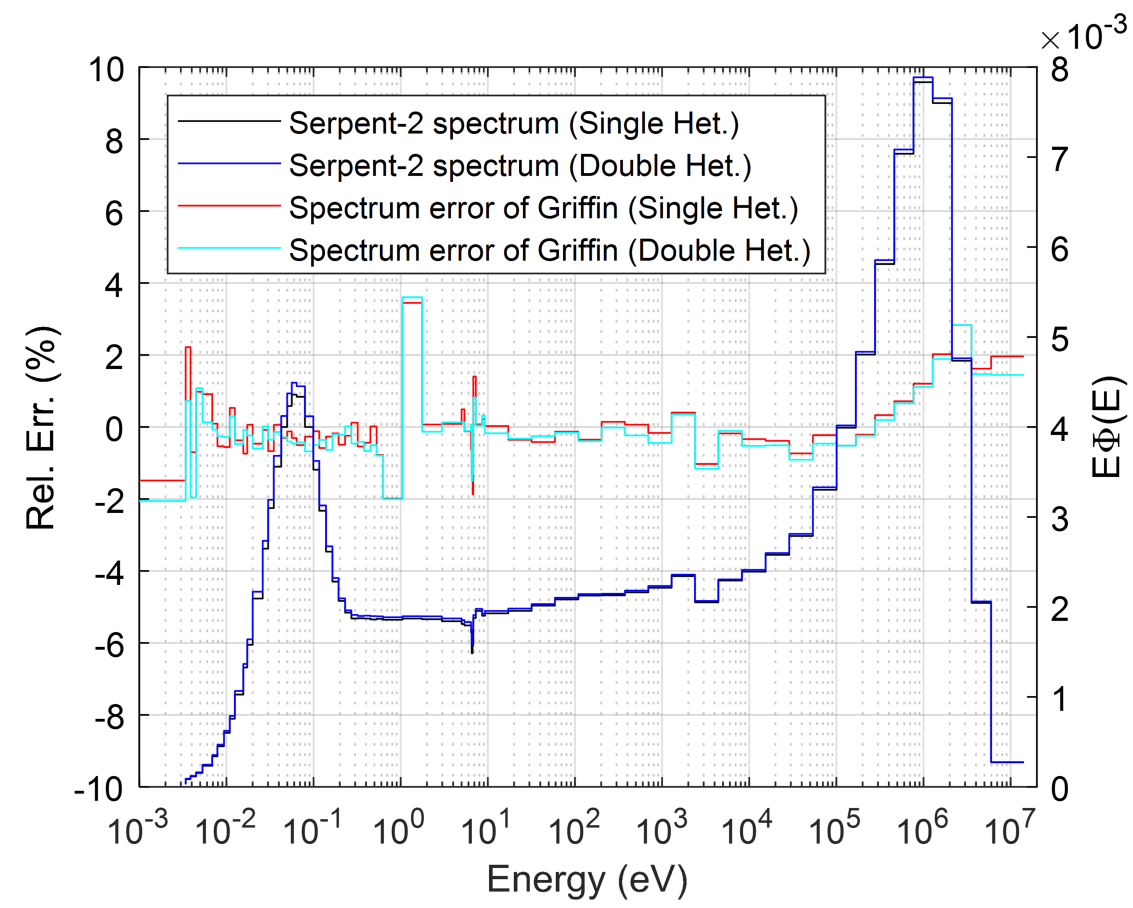

Figure 18: Serpent2 spectrum of the fuel compact and the relative error (\%) of Griffin spectrum.

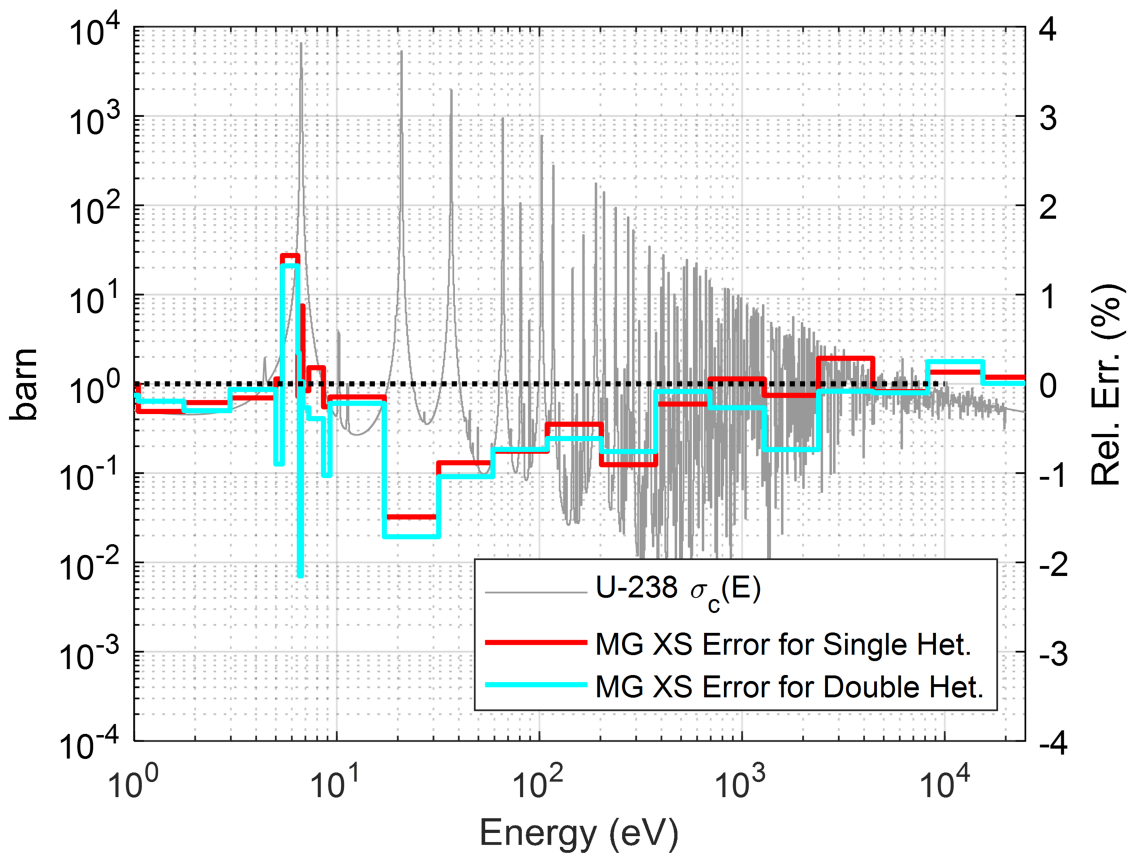

Figure 19: Relative errors of compact-averaged multigroup capture cross sections of U-238 in the fuel compact. 


\subsection{HTTR Assembly Problems}

The Griffin CSAPI was verified using the $2 \mathrm{D}$ assembly problems derived from the HTTR configuration [71]. The assembly configuration selected for verification is shown in Fig. 20 with the meshes used in the DFEM-SN calculation. It is a 31-pin $6.3 \mathrm{wt} \% \mathrm{UO}_{2}$ fuel assembly located in Zone 4 at the periphery of the core with two 2.5 wt. \% $\mathrm{B}_{4} \mathrm{C} \mathrm{BP}$ rods inserted in two out of three $\mathrm{BP}$ insertion holes and a fuel handling hole at the center. In the verification tests, two problems were solved: one with two BP rods inserted and another with $\mathrm{BP}$ rods fully withdrawn. The fuel rod is the A-type fuel with a $30.0 \%$ packing fraction of TRISO particles $(13,000$ particles with a radius of $0.046 \mathrm{~cm}$ in a 3.9-cm-high annular compact with an inner radius of $0.5 \mathrm{~cm}$ and outer radius of $1.3 \mathrm{~cm}$ ). A room temperature of $293.6 \mathrm{~K}$ was assumed for all problems.

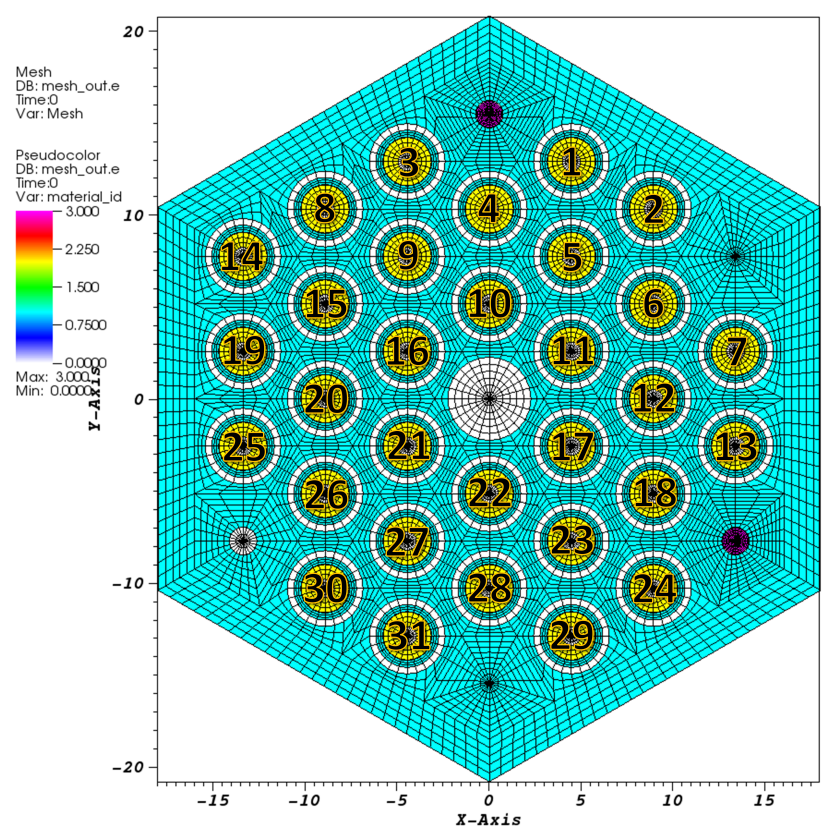

Figure 20: Exodus mesh for the HTTR 2D assembly problem of $\mathrm{B}_{4} \mathrm{C}$ rodded case.

For Serpent2 reference solutions, 31 different random particle locations were used in each fuel compact and randomly shuffled 10 times for 10 independent runs of Serpent2, and the averaged value for 10 results was taken as the reference solution. For each run of the double heterogeneous problems, $10^{5}$ particles/cycle, 100 active and 100 inactive cycles were used, and for the single heterogeneous problems, $10^{5}$ particles/cycle, 1000 active and 100 inactive cycles, were used. For Griffin calculations, each annular fuel compact and each $\mathrm{B}_{4} \mathrm{C}$ rod were divided into 3 and 9 radial meshes of the equal area with a single cross section zone, respectively, while the graphite sheath and block were divided into the enough number of meshes as shown 
in Fig. 20. A Dancoff factor and an EDC size of each fuel compact calculated in the Griffin CSAPI are shown in Fig. 21. A Dancoff factor and an EDC size are larger for compacts in the assembly periphery than for those around the assembly center, due to a larger amount of graphite, but it is noted that the amount of graphite in EDC 3 of compacts in the assembly periphery is much smaller than that of the graphite adjacent to those compacts.

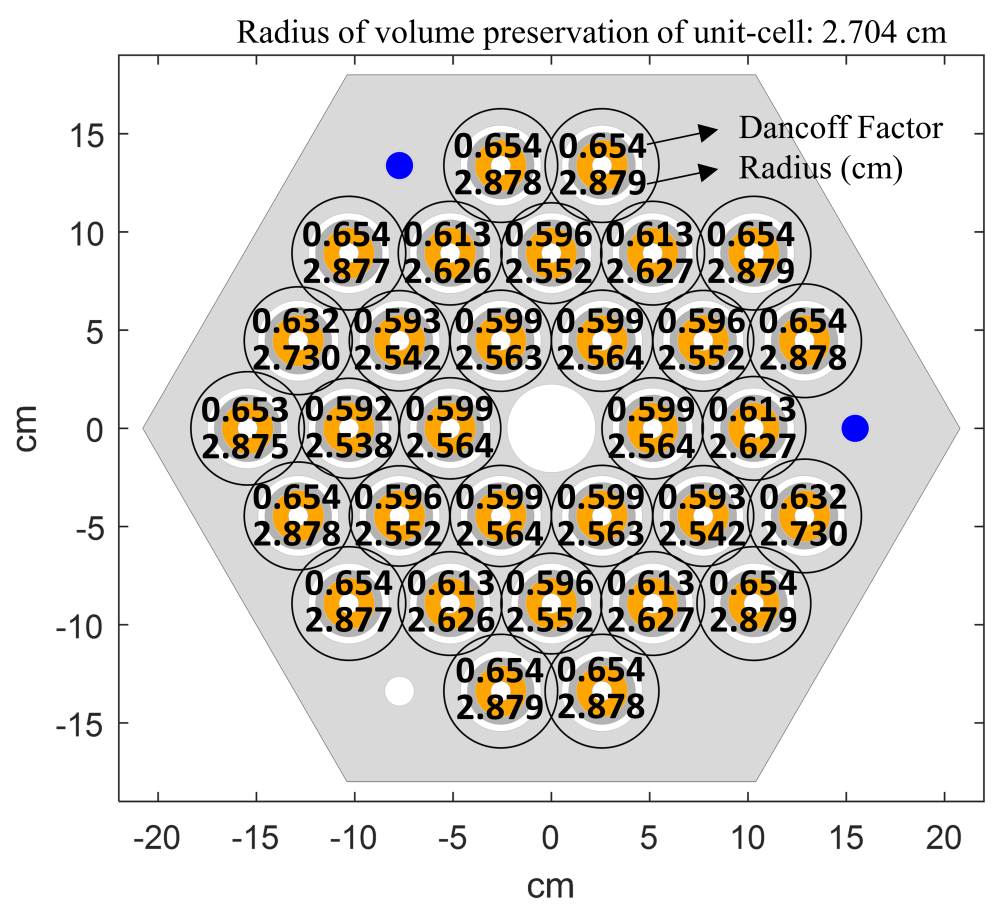

Figure 21: Dancoff factors and radii of EDC 3 of fuel compacts for 2D HTTR assembly problem.

Eigenvalue results for the single and double heterogeneous problems of $\mathrm{B}_{4} \mathrm{C}$ unrodded and rodded cases are compared in Table 13. In the Serpent 2 results for the double heterogeneous problems, there are two uncertainties: the first indicates the average value of 10 standard deviations and the second indicates the standard deviation of 10 quantities. For the power result, RMS (root mean square) and maximum values show statistics of those values among the 31 pins. For the single heterogeneous problems, the resonance table method shows eigenvalue errors of about $+240 \mathrm{pcm}$ for both cases, while the EDC method shows eigenvalue errors of +76 and +39 pcm for unrodded and rodded cases, respectively. Power distribution results of the both methods agree very well with those of Serpent2: the RMS error is about $0.12 \sim 0.14 \%$ and the maximum error is about $0.18 \sim 0.21 \%$. The EDC + ILSS method for the double heterogeneous problems shows the similar accuracy to the EDC results for the single heterogeneous problems: $+30 \sim+40$ pcm higher eigenvalue errors and less than a $0.1 \%$ difference in pin power error statistics. 
Table 13: Comparison of eigenvalue results for single and double heterogeneous problems of 2D HTTR assembly problems with and without $\mathrm{B}_{4} \mathrm{C} \mathrm{BP}$ rods.

\begin{tabular}{|c|c|c|c|c|c|c|c|c|c|}
\hline \multirow{3}{*}{\multicolumn{2}{|c|}{$\begin{array}{c}\text { Problem w/o B } \mathrm{B}_{4} \mathrm{C} \\
\text { Code/Scheme }\end{array}$}} & \multicolumn{4}{|c|}{ Single Het. } & \multicolumn{4}{|c|}{ Double Het. } \\
\hline & & \multirow{2}{*}{ K-eff } & \multirow{2}{*}{$\Delta \rho(\mathrm{pcm})$} & \multicolumn{2}{|c|}{$\Delta \mathrm{P}_{\text {pin }}(\%)$} & \multirow{2}{*}{ K-eff } & \multirow{2}{*}{$\Delta \rho(\mathrm{pcm})$} & \multicolumn{2}{|c|}{$\Delta \mathrm{P}_{\text {pin }}(\%)$} \\
\hline & & & & RMS & Max & & & RMS & Max \\
\hline \multicolumn{2}{|c|}{ Serpent 2} & 1.50820 & \pm 4 & \pm 0.04 & \pm 0.05 & 1.53375 & $\pm 13^{\mathrm{a}}$ & & $\pm 0.19^{\mathrm{c}}$ \\
\hline \multirow{2}{*}{ Griffin } & R.T. & 1.51369 & +241 & 0.14 & 0.21 & - & - & - & - \\
\hline & EDC & 1.50993 & +76 & 0.14 & 0.21 & 1.53630 & +108 & 0.14 & 0.23 \\
\hline \multicolumn{2}{|c|}{ Problem w/ $\mathrm{B}_{4} \mathrm{C}$} & \multicolumn{4}{|c|}{ Single Het. } & \multicolumn{4}{|c|}{ Double Het. } \\
\hline \multirow{2}{*}{\multicolumn{2}{|c|}{ Code/Scheme }} & \multirow{2}{*}{ K-eff } & \multirow{2}{*}{$\Delta \rho(\mathrm{pcm})$} & \multicolumn{2}{|c|}{$\Delta \mathrm{P}_{\text {pin }}(\%)$} & \multirow{2}{*}{ K-eff } & \multirow{2}{*}{$\Delta \rho(\mathrm{pcm})$} & \multicolumn{2}{|c|}{$\Delta \mathrm{P}_{\text {pin }}(\%)$} \\
\hline & & & & RMS & $\operatorname{Max}$ & & & RMS & Max \\
\hline \multicolumn{2}{|c|}{ Serpent 2} & 1.25001 & \pm 7 & \pm 0.04 & \pm 0.04 & 1.26976 & $\begin{array}{l} \pm 19^{\mathrm{a}} \\
\pm 27^{\mathrm{b}}\end{array}$ & $\begin{array}{l} \pm 0.18^{\mathrm{c}} \\
\pm 0.19^{\mathrm{d}}\end{array}$ & $\begin{array}{l} \pm 0.19^{\mathrm{c}} \\
\pm 0.33^{\mathrm{d}}\end{array}$ \\
\hline \multirow{2}{*}{ Griffin } & R.T. & 1.25375 & +238 & 0.12 & 0.18 & - & - & - & - \\
\hline & EDC & 1.25062 & +39 & 0.12 & 0.17 & 1.27103 & +78 & 0.15 & 0.25 \\
\hline
\end{tabular}

a) Average of 10 eigenvalue standard deviations, b) Standard deviation of 10 eigenvalues

c) RMS and Max for averages of 10 standard deviations of each pin power

d) RMS and Max for standard deviations of 10 pin power values

Fig. 22 shows $\mathrm{U}-238$ capture rates per fission neutron in all pins for the $\mathrm{B}_{4} \mathrm{C}$ rodded case. That is, the flux normalization was done to produce one fission neutron in the whole problem. In this normalization, an absolute difference in capture reaction rates is approximately the reactivity error induced by the capture rate error. As shown in the values of the single heterogeneous and double heterogeneous problems, U-238 capture rates are reduced by spatial self-shielding inside particles when particles are taken into account. For the single heterogeneous problem, 8.7 and 2.8 more neutrons out of $10^{5}$ fission neutrons are captured by U-238 in each pin on average, resulting in 269 and 86 more neutrons out of the $10^{5}$ fission neutrons being captured in total for the resonance table method and the EDC method, respectively. For the double heterogeneous problem, 3.9 more neutrons out of $10^{5}$ fission neutrons are captured by U-238 in each pin on average, resulting in 121 more neutrons out of $10^{5}$ fission neutrons being captured in total for the EDC + ILSS method. These results are consistent with the eigenvalue error results. For the U-235 fission rate, the sum of their errors over all pins is very small since the total number of fission neutrons is fixed for the normalization. Fig. 23 shows the pin power distribution and relative errors (\%) for the $\mathrm{B}_{4} \mathrm{C}$ rodded case. All results agree very well to a similar extent. Fig. 24 shows the relative errors of compact-averaged multigroup U-238 capture cross sections at 11 pins of Fig. 20. For the single heterogeneous problem, cross section errors are all within $\pm 1 \%$ except for the energy group of 3-5 eV, where the cause of the error was analyzed 


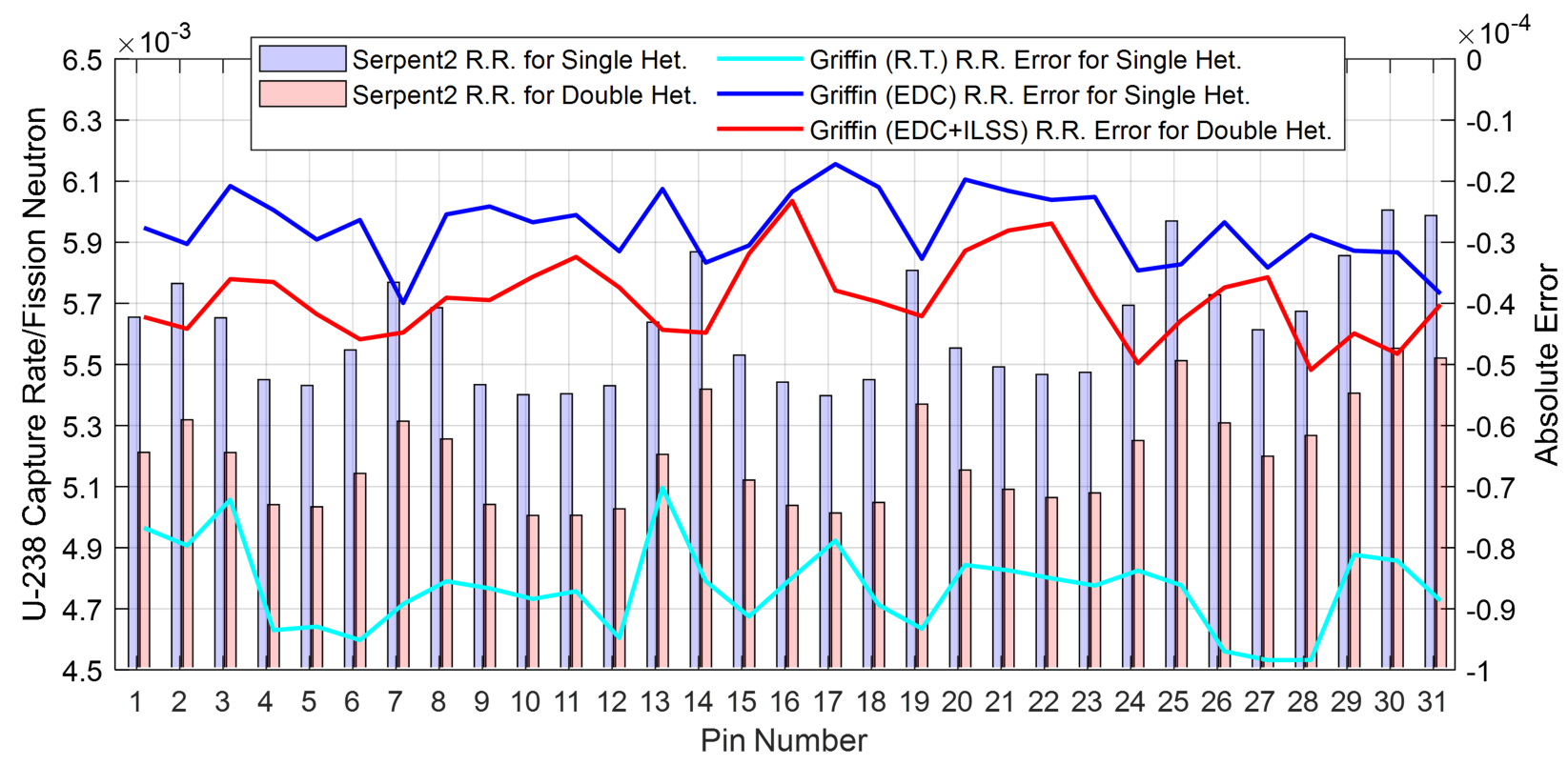

Figure 22: Comparison of U-238 capture rates per fission neutron and absolute errors for $\mathrm{B}_{4} \mathrm{C}$ rodded case (pin numbers in Fig. 20p.

in Section 4.1. For the double heterogeneous problem, cross section errors are at a similar magnitude with those for the single heterogeneous problem, but slightly negative in a consistent manner (about $-0.5 \%$ ) between 10 and $100 \mathrm{eV}$. Large errors are shown at the $6.67 \mathrm{eV}$ resonance peak. These errors will be revisited after implementing the up-scattering scheme in the slowing down calculation in future work.

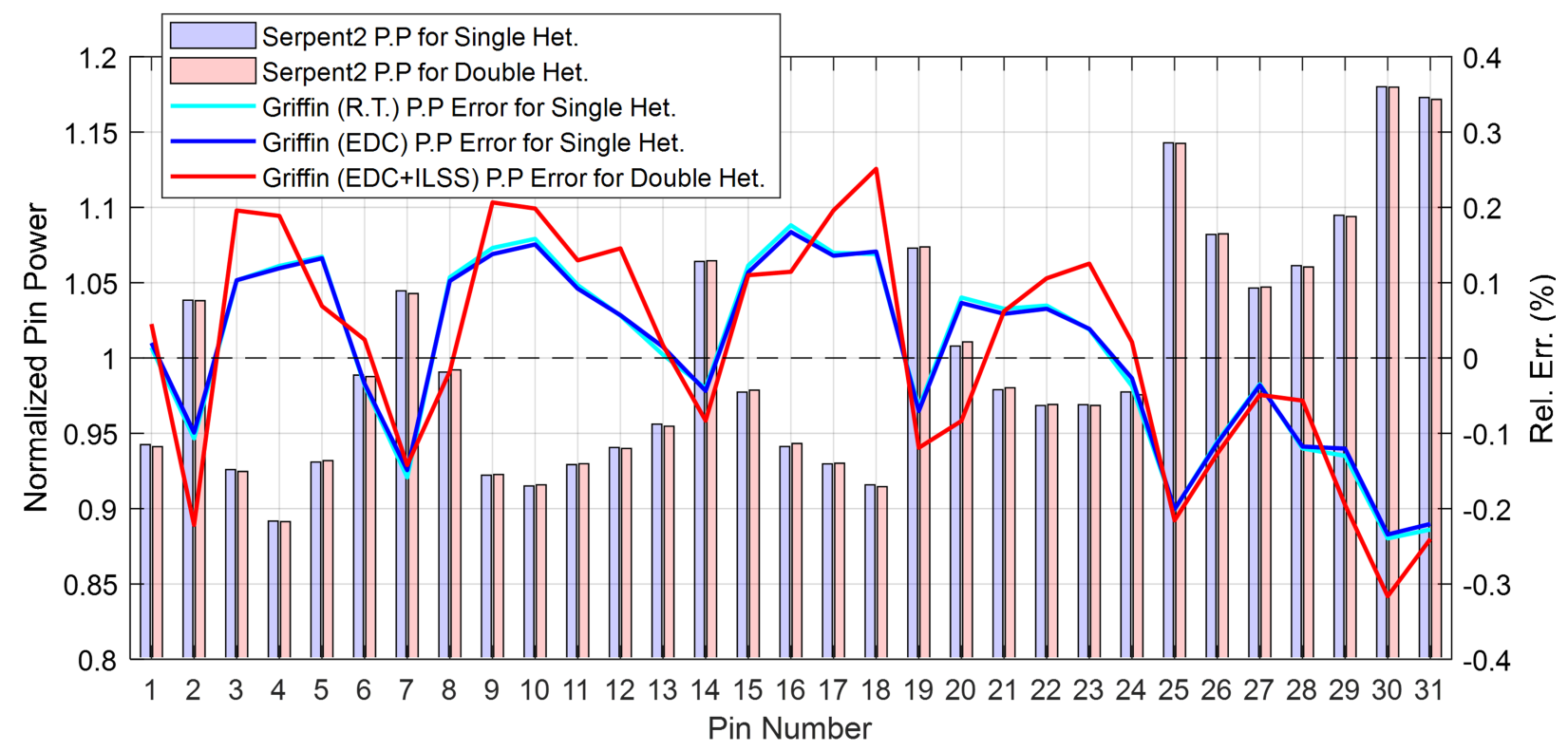

Figure 23: Comparison of pin power distribution and relative errors (\%) for $\mathrm{B}_{4} \mathrm{C}$ rodded case (pin numbers in Fig. 20p. 

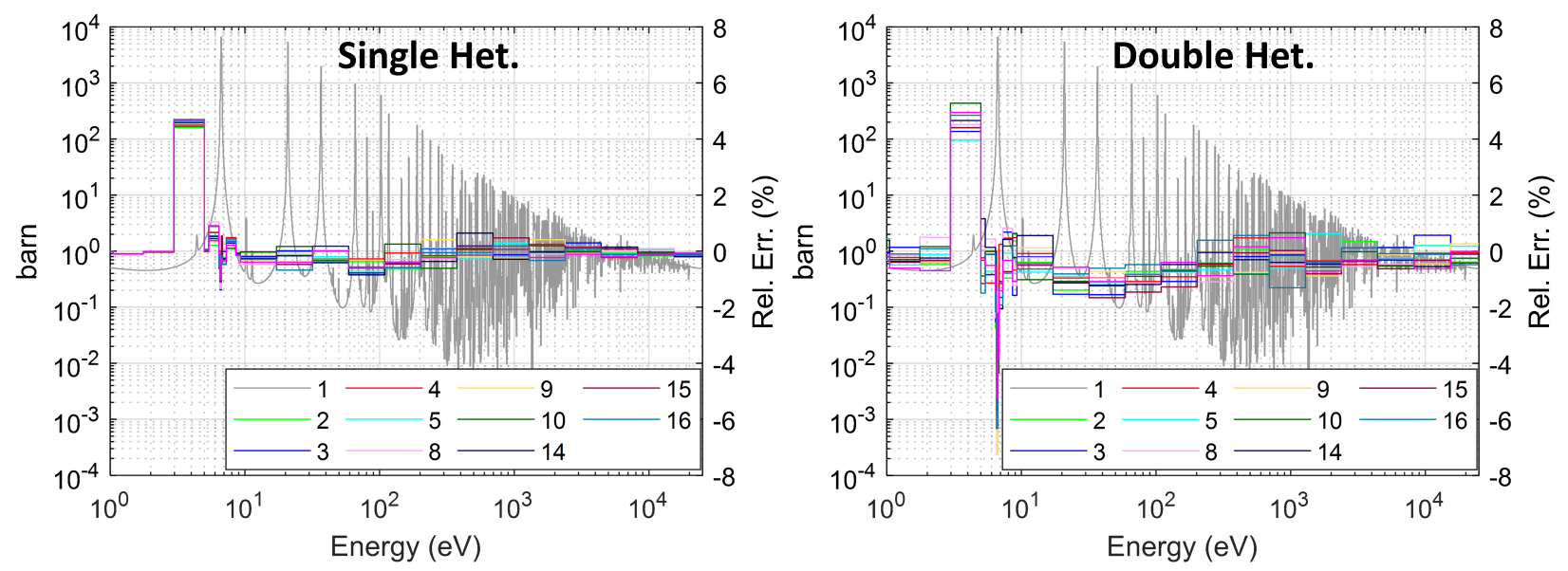

Figure 24: Relative errors of compact-averaged multigroup capture cross sections of U-238 at the selected pins in Fig. 20,

Lastly, the computational time is a concern for the online cross section generation capability. Table 14 lists the computational time of each task to solve the 2D HTTR assembly problem using 40 processors. The resonance table method took the least amount of time ( $\sim 5$ minutes) since the performance of the DFEM SN solver for FSPs has been much improved [4]. For the EDC]+ILSS method, 31 EDC 331 fuel compacts are distributed to 31 processors (nine idle processors) and the $1 \mathrm{D}$ CPM slowing down problem is solved by each of the 31 processors, which took about a minute. For the double heterogeneous problem, additional time is required to calculate $\mathrm{DF} \beta$, which takes more time $(\sim 1.5$ minutes $)$ than the $1 \mathrm{D}[\mathrm{CPM}$ slowing down calculation for the $\mathrm{EDC}$ method since $\mathrm{DF}$ iterations are involved during the slowing down calculation. The slowing down calculation scheme will be optimized in future work. In the test problem, reading the $\mathrm{MC}^{2}-3$ library took about 6 seconds, and the Dancoff factor calculation and the binary search for the EDC geometry took negligible time. Note that you may add time to reconstruct pointwise cross sections if they have to be generated on the fly. As a result, the final computational times for the single heterogeneous (using the EDC method) and the double heterogeneous problems (using the EDC + ILSS method) are about $\sim 5.5$ minutes and $\sim 7.5$ minutes, respectively. These computational times are acceptable for a pin-resolved heterogeneous assembly calculation with the 68 energy group structure. There is ample room to further reduce the computation time, which will be addressed in future work. 
Table 14: Computation time (seconds) of each task for 2D HTTR assembly problem with 68 energy groups using 40 processors.

\begin{tabular}{|c|c|c|c|}
\hline Tasks & R.T. & EDC & EDC + ILSS \\
\hline Fixed Source Problems & 23 & - & - \\
\hline Read MC ${ }^{2}-3$ Library & - & & $\leq \sim 6$ \\
\hline \begin{tabular}{|l|l|} 
HFG & Cross Section Reconstruction \\
\end{tabular} & - & & (if any) $\leq \sim 60$ \\
\hline Dancoff Factor Calculation + Binary Search for EDC & - & & $\sim 1$ \\
\hline DF Calculation & - & - & 96 \\
\hline Slowing Down for $\mathrm{DF}$ & - & & 58 \\
\hline Transport Calculation $($ DFEM $-\mathrm{SN}+\mathrm{CMFD}$ & \multicolumn{3}{|c|}{$132(14.66 \times 9)$} \\
\hline Others & 139 & 141 & 164 \\
\hline \multirow{2}{*}{ Total } & \multirow{2}{*}{294} & 338 & 457 \\
\hline & & $(+\sim 60$ & if any $\mathrm{HFG}$ XS Recon.) \\
\hline
\end{tabular}

\subsection{Pebble Fuel Problems}

The ILSS method was tested for pebble fuel problems with helium coolant. Since a significant effort is required to test pebble fuel problems using the Griffin CSAPI preliminary tests were conducted for unit pebble cell problems using the $1 \mathrm{D}$ CPM of $\mathrm{MC}^{2}-3$ with spherical geometry to evaluate the performance of the ILSS method for pebble problems. Unit pebble cell problems were derived from the HTR-10 design [72], as shown in Fig. 25. The HTR-10 design uses TRISO particles of a $17 \mathrm{wt} . \% \mathrm{UO}_{2}$ fuel kernel surrounded by graphite and $\mathrm{SiC}$ layers. The pebble is composed of a fuel zone with a $2.5 \mathrm{~cm}$ radius that has fuel particles dispersed in a graphite matrix and an outer 0.5 -cm-thick graphite layer. Eight test cases were made by changing fuel particle packing fractions from $2.4 \%$ up to $57.9 \%$.

For the Serpent 2 reference calculation, since the code can neither use a spherical surface as the outer boundary nor apply the white boundary condition, the rectangular array model with a pebble shown in Fig. 25 was constructed with the reflective boundary condition at the cube surfaces. Then, this model was converted to the $1 \mathrm{D}$ spherical model for $\mathrm{MC}^{2}-3$ by preserving the coolant volume and applying the white boundary condition. To calculate the TRISO heterogeneity effect of the models, only the fuel zone (not a whole pebble) was homogenized.

Serpent 2 calculations were performed using $10^{5}$ particles/cycle, 1000 active and 100 inactive cycles, for the single heterogeneous problem. For the double heterogeneous problem, the reference Serpent 2 solution was determined by the average of 25 Serpent 2 results obtained with different random distributions of particles in the fuel zone. Each simulation was performed using $10^{5}$ particles/cycle, 100 active and 100 inactive 


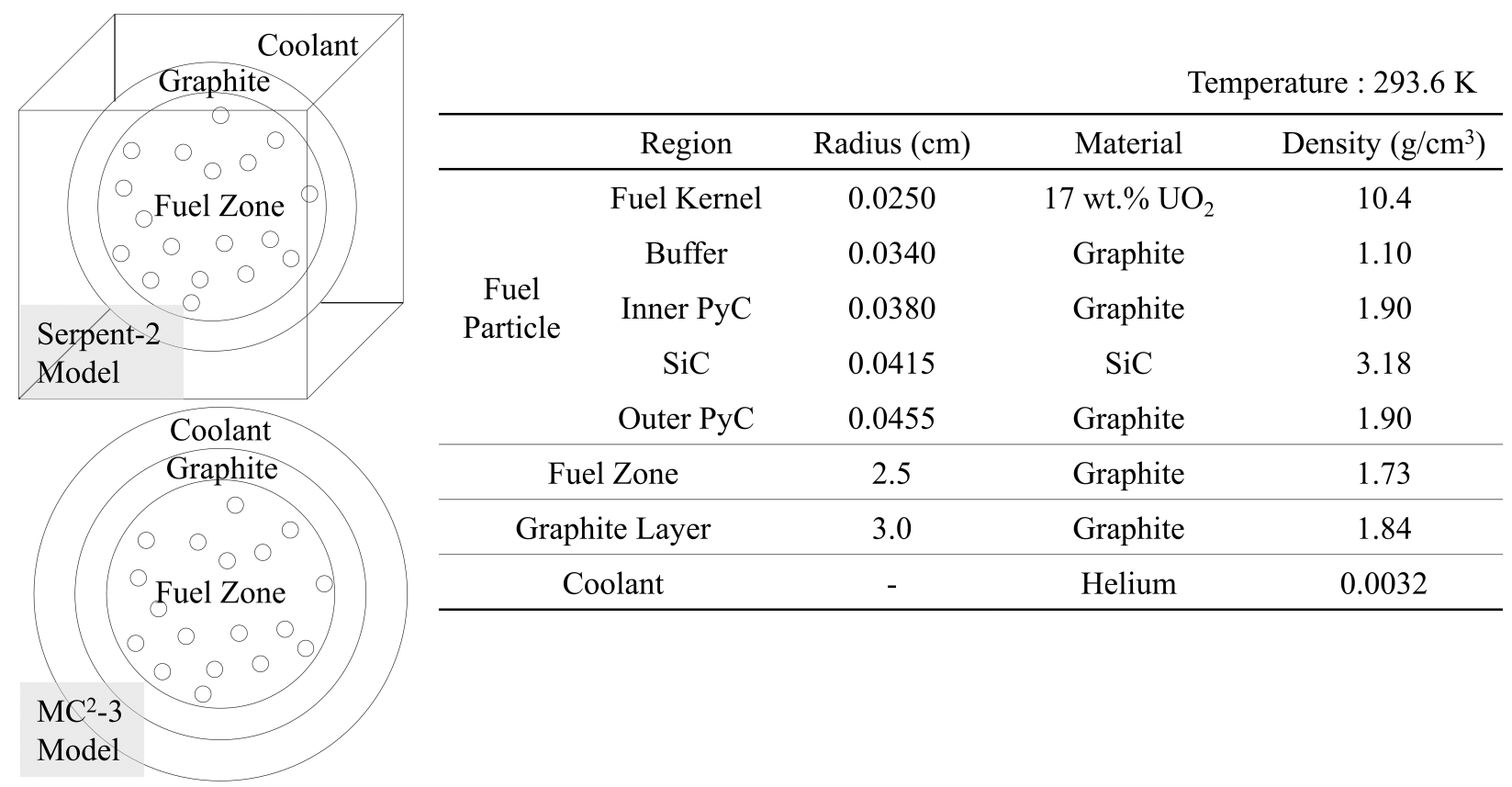

Figure 25: Pebble design obtained from HTR-10 design .

cycles. Table 15 shows the Serpent 2 results obtained for helium-cooled pebble cell problems.

$\mathrm{MC}^{2}-3$ calculations were performed using the $1 \mathrm{D}$ spherical-geometry-based $\mathrm{CPM}$ solver. In addition to the ILSS method, the different methods, including the Williams method [10], the Hébert CPM] method [73], and Sanchez-Pomraning $(\mathrm{S}-\mathrm{P}) \mathrm{CPM}$ method [74] implemented in $\mathrm{MC}^{2}-3$ were used, whose solutions were compared against Serpent 2 solutions. A sufficient number of meshes were used in the fuel zone by using a $0.1 \mathrm{~cm}$ radial thickness, and one cross section set was assigned to the entire fuel zone. The use of a larger number of cross section sets inside the fuel zone produced marginally different solutions within $20 \sim 30$

Table 15: Serpent2 results for helium-cooled pebble cell problems with different fuel particle packing fractions

\begin{tabular}{|c|c|c|c|c|c|}
\hline \multirow{2}{*}{ Packing Fraction (\%) } & \multicolumn{2}{|c|}{ Single Het. } & \multicolumn{2}{c|}{ Double Het. } & \multirow{2}{*}{$\Delta$ Het. (pcm) } \\
\cline { 2 - 5 } & K-eff & Std. & K-eff & Std. & \\
\hline 2.4 & 1.68165 & \pm 2 & 1.77111 & $\pm 7^{\mathrm{a}} / \pm 15^{\mathrm{b}}$ & 3004 \\
\hline 4.8 & 1.60023 & \pm 3 & 1.70900 & $\pm 10 / \pm 19$ & 3977 \\
\hline 9.6 & 1.46652 & \pm 5 & 1.56664 & $\pm 13 / \pm 21$ & 4358 \\
\hline 19.3 & 1.30984 & \pm 6 & 1.37811 & $\pm 17 / \pm 30$ & 3782 \\
\hline 28.9 & 1.22425 & \pm 6 & 1.27137 & $\pm 18 / \pm 22$ & 3028 \\
\hline 38.6 & 1.17461 & \pm 6 & 1.20728 & $\pm 18 / \pm 25$ & 2304 \\
\hline 48.2 & 1.14615 & \pm 6 & 1.16993 & $\pm 18 / \pm 24$ & 1773 \\
\hline 57.9 & 1.13050 & \pm 5 & 1.14869 & $\pm 18 / \pm 14$ & 1401 \\
\hline
\end{tabular}

a) Average of 25 eigenvalue standard deviations, b) Standard deviation of 25 eigenvalues 
$\mathrm{pcm}$. The exception is the S-PCPM that has limitations on the mesh size and magnitude of cross sections. Since even one mesh for the entire fuel zone resulted in a negative collision probability for some $\mathrm{HFG}$ where cross sections are too large, only one mesh for the fuel zone was used in the S-P CPM.

Table 16 compares the $\mathrm{MC}^{2}-3$ results obtained with the four methods (Williams, ILSS, Hébert, and S-P methods) with reference Serpent2 solutions for the helium-cooled problems. As shown in the table, the single heterogeneous problem results agree well with Serpent2 results within $41 \mathrm{pcm}$. The TRISO heterogeneity effect errors of the ILSS method are within $117 \mathrm{pcm}$ of the corresponding Serpent2 solutions. It was shown that all other methods (Williams, Hébert, and $\mathrm{S}-\mathrm{P}$ methods) yielded negative biases in the TRISO heterogeneity effect. The results indicate that the ILSS method works well for pebble fuel problems.

Table 16: Differences (pcm) in eigenvalue and TRISO heterogeneity effect between $\mathrm{MC}^{2}-3$ and Serpent2 solutions for helium-cooled pebble cell problems.

\begin{tabular}{|c|c|c|c|c|c|c|c|c|c|}
\hline \multirow{4}{*}{ Packing Fraction (\%) } & \multirow{3}{*}{ Single Het. } & \multicolumn{8}{|c|}{ Double Het. } \\
\hline & & \multicolumn{8}{|c|}{ Methods in $\mathrm{MC}^{2}-3$} \\
\hline & & \multicolumn{2}{|c|}{ Williams } & \multicolumn{2}{|c|}{ ILSS } & \multicolumn{2}{|c|}{ Hébert } & \multicolumn{2}{|c|}{${\mathrm{S}-\mathrm{P}^{\mathrm{p}}}^{\mathrm{s}}$} \\
\hline & $\Delta \rho$ & $\Delta \rho$ & $\Delta$ Het. & $\Delta \rho$ & $\Delta$ Het. & $\Delta \rho$ & $\Delta$ Het. & $\Delta \rho$ & $\Delta$ Het. \\
\hline 2.4 & 3 & -19 & -22 & 10 & 7 & -28 & -31 & -35 & -39 \\
\hline 4.8 & 2 & -56 & -58 & 18 & 17 & -49 & -50 & -74 & -75 \\
\hline 9.6 & -14 & -131 & -116 & 25 & 39 & -88 & -73 & -156 & -141 \\
\hline 19.3 & -12 & -219 & -208 & 23 & 35 & -145 & -133 & -283 & -271 \\
\hline 28.9 & -27 & -239 & -211 & 24 & 52 & -167 & -140 & -341 & -313 \\
\hline 38.6 & -30 & -162 & -132 & 86 & 117 & -106 & -75 & -282 & -252 \\
\hline 48.2 & -41 & -160 & -119 & 61 & 102 & -119 & -78 & -270 & -229 \\
\hline 57.9 & -38 & -164 & -127 & 25 & 62 & -136 & -98 & -231 & -194 \\
\hline
\end{tabular}

a) Only one mesh for the fuel zone was used.

In the next fiscal year, pebble-bed problems as well as these unit pebble cell problems will be simulated using the Griffin CSAPI, which will be coupled with the Pebble Tracking Transport [PTT] module [75] in Griffin. The Dancoff factor of a fuel zone of each pebble will be obtained by using the EDC method coupled with the PTT method, and an EDC of each fuel zone will be constructed. For each EDC, the EDC + ILSS method calculations will be performed to obtain fuel-zone averaged cross sections which will be provided to the PTT solver for the transport calculation. 


\section{Conclusions}

In this fiscal year, significant efforts were made to improve the capability of multigroup cross section generation in Griffin. Previously, multigroup cross sections were provided from an ISOXML-format external file that was generated from deterministic or Monte Carlo cross section generation codes for fast and thermal reactor problems. Last year, the implementation of the cross section generation capability in Griffin was initiated by plugging in CSAPI and reviewing the methodologies for treating particulate fuels.

The focus this year was on completing the CSAPI modules and implementing advanced self-shielding methods for applications to reactor problems with TRISO fuel. First, the process for cross section library generation was updated to accurately and rigorously produce isotopic cross section data. Second, the onthe-fly slowing down method (the EDC method) for the resonance treatment was implemented in CSAPI to improve the accuracy of effective multigroup cross sections in the resonance energy range. Third, the ILSS method was implemented under the calculation framework of the EDC method to accurately treat the double heterogeneity effect of particulate fuel.

The updated CSAPI with the advanced self-shielding methods, together with the improved cross section libraries, were verified against Serpent2 for graphite-moderated reactor problems including a heat-pipecooled microreactor problem. For comparison with accurate reference solutions, the stochastic dispersion of particles for double heterogeneous problems was taken into account in Serpent2 results. Eigenvalue results for all tested problems, including the VHTR pin-cell problems with a wide range of fuel particle and compact parameters, the HTTR assembly problems with and without BP rods, and the Empire unitcell problem, agreed well with Serpent2 results within $200 \mathrm{pcm}$. Detailed multigroup cross sections also agreed well within $0.5 \%$ for the VHTR problems, $1 \%$ for all fuel pins in the HTTR assembly problem, and $2 \%$ for the EMPIRE problem in the energy groups above $10 \mathrm{eV}$. Relatively larger errors were observed for the thermal energy groups below $10 \mathrm{eV}$ mainly because of the neglect of thermal up-scattering in the slowing down calculation, which will be addressed and resolved in the next fiscal year. Pin power results of the HTTR problem agreed well within $0.3 \%$. As a preliminary work, the double heterogeneity treatment methodology of the Griffin CSAPI was also verified for the helium-cooled unit-pebble cell problem from HTR-10, using the $\mathrm{MC}^{2}-3$ code, demonstrating that the ILSS method works well for pebble fuel problems. Further improvements and verification tests will be performed next year, which are summarized in the next section. 


\section{Future Work}

The initial implementation of CSAPI in Griffin was successful. However, there are many remaining tasks in order to deal with various advanced reactor problems. The recommended future work entails:

- Application of the EDC+ILSS method for pebble fuel problems to the PTT solver.

- Thermal up-scattering up to $400 \mathrm{k}_{B} \mathrm{~T}(\sim 10.36 \mathrm{eV}$ at $300 \mathrm{~K})$ and resonance up-scattering both in the slowing down calculation for the EDC method and the multigroup scattering matrix in the CSAPI multigroup library.

- Multigroup scattering matrix that accounts for problem-dependent spectrum, which requires to update the CSAPI multigroup library with additional parameters.

- Extension of the $\overline{\text { EDC }}$ method to non-fuel pins to eliminate the need for solving the $\overline{\text { FSP }}$ for non-fuel pins with resonant isotopes.

- Equivalence parameters to reduce condensation error arising from pointwise to multigroup calculations.

- Investigation of the underestimation of capture cross sections in U-238 resonances for the Empire benchmark.

- Investigation of the validity of the Dancoff factor calculation scheme for 3D problems.

- Reduction in the computation time and optimization of the $1 \mathrm{D} \mid \mathrm{CPM}$ slowing down calculation schemes.

- Rewriting the CSAPI multigroup library in the ISOXML format.

- Integration of the Shift Monte Carlo code [28] into the CSAPI multigroup library generation process.

- Investigation of the validity of the particle cell model to compute DFs in the thermal range in the presence of low-lying resonances.

- Projection and prolongation methods for homogenization and condensation in spatial and energy domains for full core calculations coupled to self-shielding methods. 


\section{REFERENCES}

[1] C. H. Lee, J. Ortensi, et al., "Griffin software development plan," Tech. Rep. ANL/NSE-21/23, INL/EXT-21-63185, Argonne National Laboratory and Idaho National Laboratory, June 2021.

[2] “Private communication with Y. Wang," September 2021.

[3] I. Diber and E. E. Lewis, "Variational nodal methods for neutron transport," Nuclear Science and Engineering, vol. 91, p. 132, 1985.

[4] Y. Wang, C. H. Lee, Y. S. Jung, Z. Prince, J. Hanophy, L. Harbour, H. Park, and J. Ortensi, "Performance improvements to the Griffin transport solvers," Tech. Rep. INL/EXT-21-64272, ANL/NSE21/51, Idaho National Laboratory and Argonne National Laboratory, September 2021.

[5] Y. Wang, S. Schunert, J. Ortensi, V. Laboure, M. DeHart, Z. Prince, F. Kong, J. Harter, P. Balestra, and F. Gleicher, "Rattlesnake: A moose-based multiphysics multischeme radiation transport application," Nuclear Technology, vol. 207, no. 7, pp. 1047-1072, 2021.

[6] C. H. Lee and Y. S. Jung, "Verification of the cross section library generated using OpenMC and MC² 3 for PROTEUS," in PHYSOR 2018: Reactors Physics paving the way towards more efficient systems, (Cancun, Mexico), April 2018.

[7] Paul K. Romano et al., "OpenMC: A state-of-the-art Monte Carlo code for research and development," Annals of Nuclear Energy, vol. 82, pp. 90-97, September 2015.

[8] J. Leppänen, "Serpent - a Continuous-energy Monte Carlo Reactor Physics Burnup Calculation Code," tech. rep., VTT Technical Research Centre of Finland, 2015.

[9] R. E. MacFarlane, D. W. Muir, R. M. Boicourt, and A. C. Kahler, "The NJOY nuclear data processing system, version 2012," tech. rep., Los Alamos National Laboratory, 2012.

[10] M. L. Williams, "Resonance Self-shielding Methodologies in SCALE 6," Nuclear Technology, vol. 174, pp. 149-168, 2011.

[11] K. Yamaji, H. Koike, Y. Kamiyama, K. Kirimura, and S. Kosaka, "Ultra-fine-group Resonance Treatment Using Equivalent Dancoff-factor Cell Model in Lattice Physics Code Galaxy," Journal of Nuclear Science and Technology, vol. 55, pp. 756-780, 2018. 
[12] H. Park and W. S. Yang and C. H. Lee, "Iterative local spatial self-shielding method of $\mathrm{MC}^{2}-3$ for particulate fuel modeling," in International Conference on Mathematics and Computational Methods Applied to Nuclear Science and Engineering (M\&C 2021), ANS, October 2021.

[13] C. H. Lee and W. S. Yang, "MC 2 -3: Multigroup cross section generation code for fast reactor analysis," Nucl Sci Eng, vol. 187, pp. 268-290, 2017.

[14] M.B. Chadwick et al., "ENDF/B-VII.1 nuclear data for science and technology: Cross sections, covariances, fission product yields and decay data," Nuclear Data Sheets, vol. 112, no. 12, pp. 2887-2996, 2011-12.

[15] K. Shibata et al., "JENDL-4.0: A New Library for Nuclear Science and Engineering," Journal of Nuclear Science and Technology, vol. 48, no. 1, pp. 1-30, 2011.

[16] Z. Ge, Y. Zhuang, T. Liu, J. Zhang, H. Wu, Z. Zhao, and H. Xia, "The upyeard version of chinese evaluated nuclear data library (CENDL-3.1)," Journal of the Korean Physical Society, vol. 59, p. 1052, 2011.

[17] A. Blokhin, A. Ignatyuk, V. Manokhin, M. Nikolaev, and V. Pronyaev, "BROND-2.2, russian evaluated neutron reaction data library," Tech. Rep. IAEA-NDS-90 Rev.8, International Atomic Energy Agency, 1984.

[18] A. Koning, R. Forrest, M. Kellett, R. Mills, H. Henriksson, and Y. Rugama, "The JEFF-3.1 nuclear data library," Research Report 6190, Nuclear Energy Agency, Organisation for Economic Co-operation and Development, 2006.

[19] The Members of the Cross Section Evaluation Working Group, "ENDF-6 formats manual," Research Report 44945-05-Rev, National Nuclear Data Center, Brookhaven National Laboratory, 2005.

[20] R. E. MacFarlane, D. W. Muir, R. M. Boicourt, A. C. Kahler, J. L. Conlin, and W. Haeck, "The NJOY nuclear data processing system, version 2016," Tech. Rep. LA-UR-17-20093, Los Alamos National Laboratory, 2016.

[21] M. E. Dunn and N. M. Greene, “AMPX-2000: Cross-section processing system for generating nuclear data for criticality safety applications," Transactions of the American Nuclear Society, vol. 86, pp. 118119, 2002. 
[22] J.-C. Sublet, P. Ribon, and M. Coste-Delclaux, “CALENDF-2002: User manual,” Tech. Rep. CEA-R6020, ISSN 0429-3460, The French Alternative Energies and Atomic Energy Commission, 2003.

[23] K. TADA, S. KUNIEDA, and Y. NAGAYA, "Nuclear Data Processing Code FRENDY Version 1," Tech. Rep. DOI:10.11484/jaea-data-code-2018-014, Japan Atomic Energy Agency, 2018.

[24] D. E. Cullen, "PREPRO 2019, ENDF/B pre-processing codes,” tech. rep., IAEA-NDS-0229, 2019.

[25] G. Marleau, A. Hébert, and R. Roy, “A User Guide for Dragon,” Tech. Rep. IGE-174 Rev. 5, Polytechnique Montréal, 2000.

[26] M. D. DeHart, TRITON: A Two-Dimensional Transport and Depletion Module for Characterization of Spent Nuclear Fuel. ORNL/TM-2005/39, Version 5.1, Vol. I, Book 3, Section T1 ed., Nov 2006.

[27] X.-. M. C. Team, “MCNP - A General Monte Carlo N-Particle Transport Code, Version 5,” Tech. Rep. LA-13709-M, Los Alamos National Laboratory, 2003.

[28] T. M. Pandya, S. R. Johnson, T. M. Evans, G. G. Davidson, S. P. Hamilton, and A. T. Godfrey, "Implementation, Capabilities, and Benchmarking of Shift, a Massively Parallel Monte Carlo Radiation Transport Code," J. Comp. Phys., vol. 308, pp. 239-272, 2016.

[29] D. K. Parsons, ANISN/PC Manual. EG\&G Idaho, Inc., December 1988.

[30] R. D. O’Dell, “Standard interface files and procedures for reactor physics codes, version IV," Tech. Rep. LA-6941-MS, Los Alamos Scientific Laboratory, 1977.

[31] "http://www.w3.org/xml/.",

[32] "http://www.unidata.ucar.edu/downloads/netcdf/index.jsp."

[33] "http://rapidxml.sourceforge.net/index.htm."

[34] I. I. Bondarenko, Group Constants for Nuclear Reactor Calculations. Constants Bureua, New York, 1964.

[35] R. J. Stamm'ler and M. J. Abbate, Methods of Steady State Reactor Physics in Nuclear Design. London: Academic Press, 1983. 
[36] S. Choi, H. Lee, S. G. Hong, and D. Lee, "Resonance self-shielding methodology of new neutron transport code STREAM,' J. Nucl. Sci. Technol., vol. 52, pp. 1133-1150, 2015.

[37] H. G. Joo, G. Y. Kim, and L. Pogosbekyan, "Subgroup weight generation based on shielded pin-cell cross section conservation," Ann. Nucl. Energy, vol. 36, pp. 859-868, 2009.

[38] S. G. Hong and K. S. Kim, "Iterative resonance self-shielding methods using resonance integral table in heterogeneous transport lattice calculations," Ann. Nucl. Energy, vol. 38, pp. 32-43, 2011.

[39] M. L. Williams and K.-S. Kim, "The embedded self-shielding method," in PHYSOR 2012 - Advances in Reactor Physics - Linking Research, Industry, and Education, American Nuclear Society, April 2012.

[40] R. J. Stamm'ler, “HELIOS-2 methods,” tech. rep., Studsvik Scandpower, 1998.

[41] J. Y. Cho, K. S. Kim, H. Y. Kim, C. C. Lee, S. Q. Zee, and H. G. Zoo, “DeCART v1.2 user's manual,” Tech. Rep. KAERI/TR-3438/2007, 2007.

[42] Y. S. Jung, C. B. Shim, C. H. Lim, and H. G. Joo, "Practical numerical reactor employing direct whole core neutron transport and subchannel thermal/hydraulic solvers," Ann. Nucl. Energy, vol. 62, pp. 357-374, 2013.

[43] “MPACT Theory Manual Version 2.2.0,” Tech. Rep. CASL-U-2016-1107-000, 2016.

[44] Y. Liu and W. R. Martin, "Assessment of homogeneous and heterogeneous resonance integral tables and their applications to the embedded self-shielding method," Ann. Nucl. Energy, vol. 92, pp. 186197, 2016.

[45] T. Tone, "A numerical study of heterogeneity effects in fast reactor critical assemblies," J. Nucl. Sci. Technol., vol. 12, pp. 467-481, 1975.

[46] N. Sugimura and A. Yamamoto, "Resonance treatment based on ultra-fine-group spectrum calculation in the AEGIS code," J. Nucl. Sci. Technol., vol. 44, pp. 958-966, 2007.

[47] Y. Liu, W. Martin, M. Williams, and K. S. Kim, "A full core resonance self-shielding method using a continuous-energy quasi-one-dimensional slowing-down solution that accounts for temperaturedependent fuel subregions and resonance interference," Nucl. Sci. Eng., vol. 180, pp. 247-272, 2017. 
[48] S. Choi, C. Lee, and D. Lee, "Resonance treatment using pin-based pointwise energy slowing-down method," J. Comput. Phys., vol. 330, pp. 134-155, 2017.

[49] A. Yamamoto, "Evaluation of background cross section for heterogeneous and complicated geometry by the enhanced neutron current method," J. Nucl. Sci. Technol., vol. 45, pp. 1287-1292, 2008.

[50] IAEA, “WIMS-D Library Update,” Tech. Rep. ISBN 92-0-105006-2, 2007.

[51] C. A. Wemple, R. J. J. Stamm'ler, and A. A. Ferri, "Improved temperature-dependent resonance treatment in HELIOS-1.9," Transactions of the American Nuclear Society, vol. 96, pp. 657-659, 2009.

[52] Y. S. Jung, C. H. Lim, and H. G. Joo, “Temperature dependent subgroup formulation with number density adjustment for direct whole core power reactor calculation,” Ann. Nucl. Energy, vol. 96, pp. 249$263,2016$.

[53] M. Ouisloumen and R. Sanchez, "A model for neutron scattering off heavy isotopes that accounts for thermal agitation effects," Nucl. Sci. Eng., vol. 107, pp. 189-200, 1991.

[54] D. Lee, K. Smith, and J. Rhodes, "The impact of U-238 resonance elastic scattering approximations on thermal reactor Doppler reactivity," Ann. Nucl. Energy, vol. 36, pp. 274-280, 2009.

[55] R. Dagan, "On the use of $\mathrm{s}(\alpha, \beta)$ tables for nuclides with well pronounced resonances," Ann. Nucl. Energy, vol. 32, pp. 367-377, 2005.

[56] T. Viitanen and J. Leppänen, "Target motion sampling temperature treatment technique with elevated basis cross-section temperatures," Nucl. Sci. Eng., vol. 177, pp. 77-89, 2014.

[57] W. Rothenstein, "Neutron scattering kernels in pronounced resonances for stochastic doppler effect calculations," Ann. Nucl. Energy, vol. 23, pp. 441-458, 1996.

[58] K. S. Kim, "Procedure to generate the mpact multigroup library," Tech. Rep. CASL-X-2015-1013-000, December 2015.

[59] A. Gibson, Novel Resonance Self-shielding Methods for Nuclear Reactor Analysis. PhD thesis, Massachusetts Institute of Technology, 2016. 
[60] H. Park and H. G. Joo, "Practical resolution of angle dependency of multigroup resonance cross sections using parametrized spectral superhomogenization factors," Nucl. Eng. Technol., vol. 49, pp. 1287-1300, 2017.

[61] G. I. Bell, G. E. Hansen, and H. A. Sandmeier, "Multitable treatments of anisotropic scattering in sn multigroup transport calculation,” Nucl. Sci. Eng., vol. 28, pp. 376-383, 1967.

[62] A. Hébert, "Advances in the development of a sub-group method for the self-shielding of resonant isotopes in arbitrary geometries," Nucl. Sci. Eng., vol. 126, pp. 245-263, 1997.

[63] K. S. Kim, M. L. Williams, D. Wiarda, K. T. Clarno, and Y. Liu, "Development of the CASL-VERA V4.2m5 MPACT 51-group Libraries with ENDF/B-VII.0 and VII.1," in International Conference on Mathematics and Computational Methods Applied to Nuclear Science and Engineering (M\&C 2017), ANS, April 2017.

[64] G. Giudicelli, K. Smith, and B. Forget, "Generalized equivalence methods for 3D multi-group neutron transport," Ann. Nucl. Energy, vol. 112, pp. 9-16, 2018.

[65] L. Mao, I. Zmijarevic, and K. Routsonis, "Application of the SPH method to account for the angular dependence of multigroup resonant cross sections in thermal reactor calculations," Ann. Nucl. Energy, vol. 124, pp. 98-118, 2019.

[66] H. Park, Resonance Treatment Innovations for Efficiency and Accuracy Enhancement in Direct Whole Core Calculations of Water Cooled Power Reactors. PhD thesis, Seoul National University, 2018.

[67] J. Rhodes, K. Smith, and D. Lee, "CASMO-5 development and applications," in PHYSOR-2006, ANS Topical Meeting on Reactor Physics, Organized and hosted by the Canadian Nuclear Society, (Vancouver, BC, Canada), Sept. 2006.

[68] Y. S. Jung and C. H. Lee, "PROTEUS-MOC User Manual," Technical Report ANL/NE-18/10, Argonne National Laboratory, September 2018.

[69] C. Matthews, V. Laboure, J. Ortensi, M. DeHart, Y. Wang, and R. C. Martineau, "Evaluation of the MOOSE Tool-set for Analysis of Thermo-Mechanical-Neutronics coupling in Micro-Reactors," Tech. Rep. Los Alamos National Laboratory, LA-UR-19-31443, 2019. 
[70] N. E. Stauff, K. Mo, C. Lee, Z. Mei, Y. S. Jung, C. Matthews, and B. Feng, "Multi-physics simulations of heat-pipe triso-fueled micro-reactor," Transactions of the American Nuclear Society, vol. 123, pp. 1383-1385, 2020.

[71] J. D. Bess and N. Fujimoto, "Evaluation of the Start-Up Core Physics Tests at Japan's High Temperature Engineering Test Reactor (Fully Loaded Core),” Tech. Rep. HTTR-GCR-RESR-001,International Handbook of Evaluated Reactor Physics Benchmark Experiments, NEA/NSC/DOC(2006)1, OECDNEA, 2010.

[72] Z. Wu, D. Lin, and D. Zhong, “The design features of the HTR-10," Nuclear Engineering and Design, vol. 218, pp. 25-32, 2002.

[73] A. Hébert, "A collision probability analysis of the double-heterogeneity problem," Nucl. Sci. Eng., vol. 115, pp. 177-184, 1993.

[74] R. Sanchez and G. C. Pomraning, "A statistical analysis of the double heterogeneity problem," Ann. Nucl. Energy, vol. 18, pp. 371-395, 1991.

[75] Y. Wang, J. Ortensi, S. Schunert, and V. Laboure, “A pebble tracking transport algorithm for pebble bed reactor analysis," in PHYSOR 2018: Reactor Physics paving the way towards more efficient systems, (Cancun, Mexico), April 2018. 